University of Texas at El Paso

\title{
DigitalCommons@UTEP
}

Open Access Theses \& Dissertations

2018-01-01

\section{Superpixel-Based Hyperspectral Unmixing With Regional Segmentation}

Mohammed Qassim Alkhatib

University of Texas at El Paso, mqalkhatib@ieee.org

Follow this and additional works at: https://digitalcommons.utep.edu/open_etd

Part of the Remote Sensing Commons

\section{Recommended Citation}

Alkhatib, Mohammed Qassim, "Superpixel-Based Hyperspectral Unmixing With Regional Segmentation" (2018). Open Access Theses \& Dissertations. 1393.

https://digitalcommons.utep.edu/open_etd/1393

This is brought to you for free and open access by DigitalCommons@UTEP. It has been accepted for inclusion in Open Access Theses \& Dissertations by an authorized administrator of DigitalCommons@UTEP. For more information, please contact lweber@utep.edu. 


\title{
SUPERPIXEL-BASED HYPERSPECTRAL UNMIXING WITH REGIONAL SEGMENTATION
}

\author{
MOHAMMED QASSIM ALKHATIB \\ Doctoral Program in Electrical and Computer Engineering
}

APPROVED:

Miguel Velez-Reyes, Ph.D., Chair

Sergio D. Cabrera, Ph.D.

Raed AlDouri, Ph.D.

Charles Ambler, Ph.D.

Dean of the Graduate School 


\section{Copyright (C)}

by

Mohammed Q. Alkhatib

2018 


\section{DEDICATION}

This work is dedicated to my great wife for her support and help during this period of my life. To my great parents for their prayers and support. Finally, to my beautiful daughter Zaina. 


\title{
SUPERPIXEL-BASED HYPERSPECTRAL UNMIXING WITH REGIONAL SEGMENTATION \\ by
}

MOHAMMED QASSIM ALKHATIB, M.S.E.E.

\author{
DISSERTATION
}
Presented to the Faculty of the Graduate School of The University of Texas at El Paso in Partial Fulfillment of the Requirements for the Degree of

DOCTOR OF PHILOSOPHY

The Department of Electrical and Computer Engineering THE UNIVERSITY OF TEXAS AT EL PASO

May 2018 


\section{ACKNOWLEDGEMENTS}

I would like to take this opportunity to express my sincere thanks to Dr. Miguel VelezReyes for his guidance and support, which without him this would not have been possible, I'm also very thankful to him for accepting me as his research assistant which provided me with experience and financial support to help me pursue this degree.

I would also like to sincerely acknowledge and thank Dr. Sergio Cabrera for his assistance in serving in my committee, and recommending me to receive the Texas Instruments Foundation (TIF) Scholarship which provided me with financial support to help me pursue this degree. Also, I appreciate and thank Dr. Raed Aldouri, from the Department of Civil Engineering, for his advice and for serving in my committee.

I want to thank the University of Texas at El Paso and the Department of Electrical and Computer Engineering for giving me the opportunity to grow intellectually and for the economic support throughout these years. 


\begin{abstract}
Unsupervised unmixing analysis aims to extract the basic materials, also known as, endmembers, and their fractions (or abundances) from a hyperspectral image. In this work, a new unsupervised unmixing approach based on Low Dimensional representation with regional analysis is developed. A low dimensional image representation is obtained using superpixel segmentation where each superpixel is represented by its mean spectra. Regional analysis is then performed using the quadtree segmentation based on the Shannon entropy. Endmembers are extracted from each region and combined using clustering into endmember classes. The proposed approach is tested and validated using the HyDICE Urban, ROSIS Pavia, and AVIRIS Fort AP Hill data sets. Different levels of qualitative and quantitative assessments are performed based on the available reference data. The proposed approach is also compared with global (no-regional segmentation) and with pixel-based (no-superpixel dimensionality reduction) unsupervised unmixing approaches. Qualitative assessment was based primarily on agreement with spatial distribution of materials provided by a reference classification map. Quantitative assessment was based on comparing classification maps derived from abundance maps using winner takes it all and reference data. High agreements with reference data were obtained by the proposed approach as evidenced by high kappa values (over 80\%). The proposed approach outperforms all global unsupervised unmixing approaches that do not account for regional information. Performance is slightly better when compared to pixel-based approached using regional segmentation, however our approach resulted in significant computational savings due to the dimensionality reduction step.
\end{abstract}




\section{TABLE OF CONTENTS}

Page

ACKNOWLEDGMENTS ........................ . . . . .

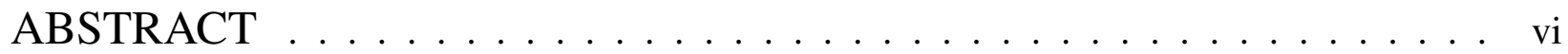

TABLE OF CONTENTS ......................... ix

LIST OF FIGURES . . . . . . . . . . . . . . . . . . . . x

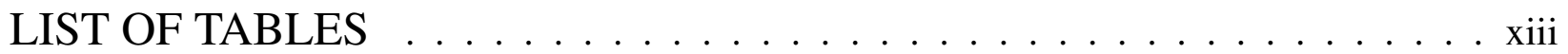

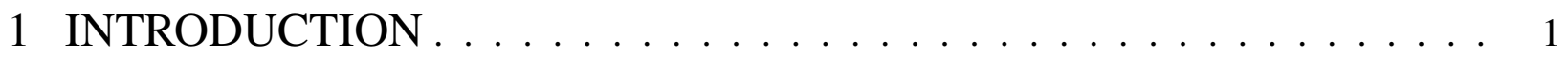

1.1 Justification . . . . . . . . . . . . . . . . . . 1

1.2 Problem Statement . . . . . . . . . . . . . . . . . . . . . 2

1.3 Technical Approach . . . . . . . . . . . . . . . . . . . . 2

1.4 Research Objectives . . . . . . . . . . . . . . . . . . . . . . . . . . . . . . . . . .

1.5 Dissertation Outline . . . . . . . . . . . . . . . . 3

2 BACKGROUND AND LITERATURE REVIEW . . . . . . . . . . . . . . . 5

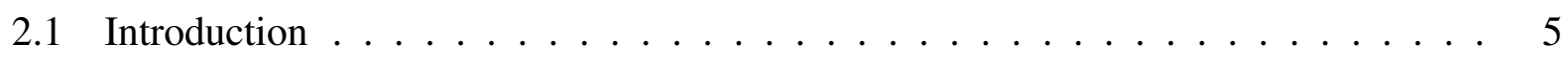

2.2 Remote Sensing and Hyperspectral Imaging . . . . . . . . . . . . . . . . . . 5

2.3 Linear Mixing Model . . . . . . . . . . . . . . . . . . . . . 8

2.3.1 Endmembers Spectral Signature . . . . . . . . . . . . . . . . . . . . 9

2.4 Spectral Unmixing . . . . . . . . . . . . . . . . . . 11

2.4.1 Endmembers Determination Approaches . . . . . . . . . . . . . 12

2.4.2 Determining the Number of Endmembers . . . . . . . . . . . . . 19

2.4 .3 Abundance Estimation . . . . . . . . . . . . . . . . . . . 22

2.5 Superpixel Segmentation . . . . . . . . . . . . . . . . . . 24

2.5.1 Simple linear iterative clustering . . . . . . . . . . . . . 25

2.6 Hyperspectral Image Segmentation . . . . . . . . . . . . . . . . . . . . . . . . . . . . . .

2.6.1 Disjunctive Approach . . . . . . . . . . . . . . . . . . 26

2.6.2 Conjunctive Approach . . . . . . . . . . . . . . . 26

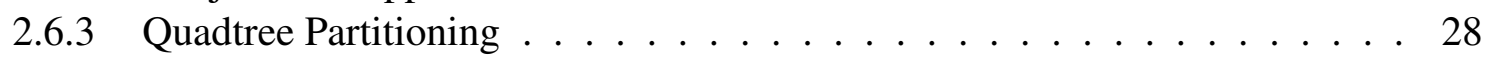

2.6 .4 Segmentation HSI Using SLIC . . . . . . . . . . . . . . . . . . . . . . . 29

2.7 Clustering Algorithms . . . . . . . . . . . . . . . . . . . . . . . . . . 29

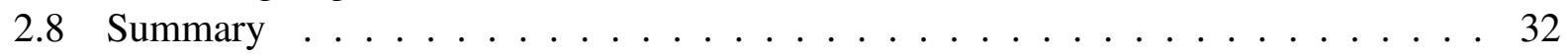

3 UNDERSTANDING SPATIAL-SPECTRAL DOMAIN INTERACTIONS IN HYPERSPECTRAL UNMIXING USING EXPLORATORY DATA

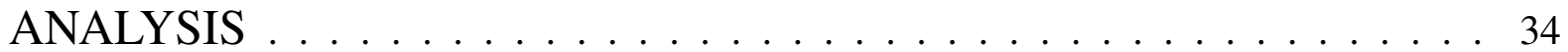

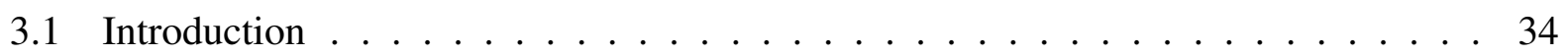

3.2 Data Cloud Image Representation . . . . . . . . . . . . . . . . 34 
3.3 Partitioning the Data Cloud . . . . . . . . . . . . . . . . . . 36

3.4 Extracting Piecewise-Convex Structure Using Spatial Segmentation . . . . . . . 37

3.5 Dimensionality Reduction Using Superpixel Segmentation . . . . . . . . . . . 37

3.6 Summary . . . . . . . . . . . . . . . . . . . . . . . . . . . 41

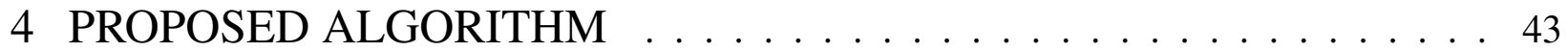

4.1 Introduction . . . . . . . . . . . . . . . . . . 43

4.2 Dimensionality Reduction Using SLIC . . . . . . . . . . . . . . . . . . . . 43

4.3 Quadtree Segmentation . . . . . . . . . . . . . . . . . . . . . 45

4.4 Endmember Extraction . . . . . . . . . . . . . . . . . . . . . . 45

4.4.1 Estimation of the Number of Endmembers Using the Gram Method . . . 45

4.4.2 Endmember Extraction Using SVDSS . . . . . . . . . . . . . . 46

4.5 Endmember Class Extraction . . . . . . . . . . . . . . . . . . . . . . 47

4.6 Abundance Estimation . . . . . . . . . . . . . . . . . . . . 47

4.7 Summary . . . . . . . . . . . . . . . . . . . . . . 48

5 EXPERIMENTS WITH REAL HSI DATA SETS . . . . . . . . . . . . . . . . 49

5.1 Introduction . . . . . . . . . . . . . . . . . . . . . . . . . . . 49

5.2 Data Sets . . . . . . . . . . . . . . . . . . . . . . . . 49

$5.2 .1 \quad$ Urban Data Set . . . . . . . . . . . . . . . . . . . . . . . . . 49

5.2 .2 Pavia University . . . . . . . . . . . . . . . . . . . . . . . . . . . . 51

5.3 Assessment Methods . . . . . . . . . . . . . . . . . . . . 51

5.3 .1 Qualitative Evaluation . . . . . . . . . . . . . . . . . 53

5.3.2 Quantitative Assessment of Detected Classes . . . . . . . . . . . . . . 54

5.4 Results . . . . . . . . . . . . . . . . . . . . . . 56

5.4 .1 Results of Urban Data Set . . . . . . . . . . . . . . . . 56

5.4.2 Results for Pavia University Data Set . . . . . . . . . . . . . . . . . 68

5.5 Comparing With Other methods . . . . . . . . . . . . . . . . . 78

5.5.1 Comparing With the Global Approach . . . . . . . . . . . . . . . . 80

5.5.2 Comparing with the Global Low Dimensional Approach with no Segmen-

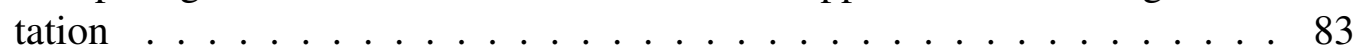

5.5.3 Comparing With the Quadtree Approach and no Superpixel Segmentation . 84

5.5 .4 Additional Comparison . . . . . . . . . . . . . . . . . . . . . . . 89

5.6 Summary . . . . . . . . . . . . . . . . . . . . . 91

6 EXPERIMENTS WITH FORT A.P. HILL DATA SET . . . . . . . . . . . . . 92

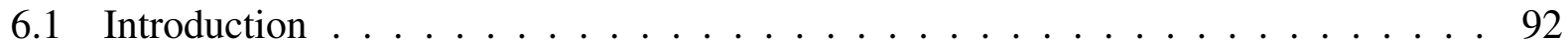

6.2 Justification . . . . . . . . . . . . . . . . . . . . . . . . . . . . 92

6.3 Creating New Reference Map . . . . . . . . . . . . . . . . . . . . . . . . . 94

6.4 Unmixing Analysis . . . . . . . . . . . . . . . . . . . . . . . . . 95

6.5 Qualitative Evaluation with Existing High Resolution Image . . . . . . . . . 101

6.6 Summary . . . . . . . . . . . . . . . . . . . . . . 102

7 CONCLUSIONS AND FUTURE WORK . . . . . . . . . . . . . . . . . . 104

7.1 Conclusions . . . . . . . . . . . . . . . . . . . . . . . . . . . . . . 104 
7.2 Future Work . . . . . . . . . . . . . . . . . . 106

REFERENCES . . . . . . . . . . . . . . . . . . . 108

CURRICULUM VITA . . . . . . . . . . . . . . . . . . . . . . . 114 


\section{LIST OF FIGURES}

1.1 Proposed segmentation-based unmixing. . . . . . . . . . . . . . 3

2.1 overview of Remote sensing system. [1] . . . . . . . . . . . . . . 6

2.2 Spectral sampling in spectral imaging. . . . . . . . . . . . . . . 6

2.3 Two mixing models. (a) The linear mixing model assumes a well-defined proportional checkerboard mixture of materials, with a single reflection of the illuminating solar radiation. (b) Nonlinear mixing models assume a randomly distributed, homogeneous mixture of materials, with multiple reflections of the illuminating radiation $[2] \ldots \ldots \ldots \ldots \ldots$

2.4 Spectral Signatures of 3 different materials. . . . . . . . . . . . . . . 10

2.5 Feature space representation of the three pure endmembers. . . . . . . . . . . 10

2.6 Feature space representation of the three mixed endmembers. . . . . . . . . . 11

2.7 Local and global effects of applying spatial-spectral dilation and erosion operations to common hyperspectral image $[3] \ldots \ldots \ldots$. . . . . . . . . . . 16

2.8 Multigrid structure $[4] \ldots \ldots \ldots \ldots \ldots$

2.9 Image Partitioning using quadtree $[5] \ldots \ldots \ldots \ldots$

2.10 Superpixel segmentation for a chip from A.P. Hill Scene . . . . . . . . . . . . 26

2.11 Example of Disjunctive Approach [6] . . . . . . . . . . . . . . . . . . . 27

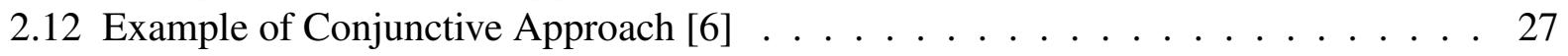

2.13 Quadtree image representation . . . . . . . . . . . . . . . . 28

2.14 Example of Dendrogram for hierarchical clustering . . . . . . . . . . . 31

3.1 (a) Endmembers used in the simulated data; (b) RGB composite for spatially random distributed data; (c) RGB composite of image with spatial structure. . . . . . . 35

3.2 (a) Feature data cloud of the first scenario (materials are randomly distributed in the scene); (b) Feature data cloud of the second scenario (materials occupy some uniform spatial regions. . . . . . . . . . . . . . . . . . 36

3.3 (a) True color RGB of a chip from AP Hill image; (b) Projection of the data points on the first 2 principal components. . . . . . . . . . . . . . . 36

3.4 (a) Segmentation of the data cloud in feature space; (b) Region 1 in the spatial domain (Gravel field); (c) Region 2 in the spatial domain (Grass field); (d) Region 3 in the spatial domain (Summer deciduous forest) . . . . . . . . . . . 38

3.5 SLIC segmentation of AP Hill chip . . . . . . . . . . . . . . . . . . 39

3.6 Result of SLIC Partitioning and their corresponding location in the cloud. . . . . . 40

3.7 (a) Projection of the data points on the first 2 principal components; (b) RGB composite of the original image; (c) Superpixel centroids in feature space (region size = 25); (d) Location of superpixel centroids on the data cloud; (e) RGB composite of the LD image; (f) Superpixel centroids in feature space (Region Size $=5$ ); (g) Location of superpixel centroids on the data cloud; (h) RGB composite of the LD

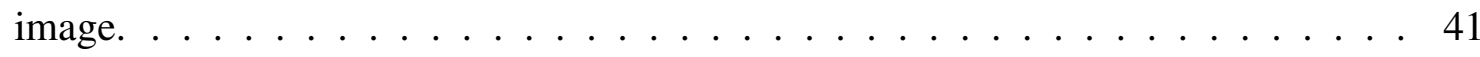

4.1 Proposed superpixel-based unmixing . . . . . . . . . . . . . . . 43 
4.2 SLIC Segmentation. . . . . . . . . . . . . . . . . . . . . . . . . . 44

5.1 Urban Image: (a) true color RGB composite; (b) Reference data classification map [7]; (c) Urban Endmembers. . . . . . . . . . . . . . . . . . . . . . . . 50

5.2 ROSIS Pavia University (a) true color composite; (b) Reference calss map; (c) Endmembers. . . . . . . . . . . . . . . . . . . . 52

5.3 SLIC image of the urban scene: (a) SLIC over-segmentation; (b) low dimensional representation, each pixel replace by the mean. . . . . . . . . . . . . . 56

5.4 Partitioning using quadtree $(\mathrm{EMs}=$ Endmembers $) \ldots \ldots \ldots \ldots$. . . . . 57

5.5 Endmembers for each tile. . . . . . . . . . . . . . . . . . 58

5.6 Spectral endmembers classes for Urban using angle distance of 0.005 . . . . . . . 59

5.7 Abundances of spectral endmember classes in Urban using proposed approach. . . 60

5.8 Resulting Urban information classes. . . . . . . . . . . . . . . . . . . 61

5.9 Resulting Urban information classes abundance maps. . . . . . . . . . . . . . . . 61

5.10 Comparison between abundance maps extracted using the proposed method and the reference data from [7]. . . . . . . . . . . . . . . . . . . . . 63

5.11 Histograms for the abundance maps in Figure 5.10 and error statistics. Pixels with value of zero were not included in the histogram plots as in most images they were the vast majority of pixels. . . . . . . . . . . . . . . . . . . . . . 64

5.12 Comparison of endmember classes extracted with the proposed algorithm and reference data spectral signatures for endmembers. . . . . . . . . . . . . . . 65

5.13 Urban Image: (a) Classification map using the abundances and the majority vote criterion; (b) Classification map after filtering; (c) Reference data classification map [7]

5.14 (a) SLIC image of the Pavia data set; (b) RGB display of the low dimensional superpixel image . . . . . . . . . . . . . . . . . . . . . 69

5.15 Pavia Partitioning using quadtree $(\mathrm{EMs}=$ Endmembers $) \ldots \ldots \ldots 70$

5.16 Endmembers for each tile. . . . . . . . . . . . . . . . . . . 71

5.17 Spectral endmembers classes for Pavia scene using angle distance of 0.005 . . . . 72

5.18 Spectral endmembers classes for Pavia scene using angle distance of 0.005. . . . 73

5.19 combining similar spectra of the Pavia Scene. . . . . . . . . . . . . . . 74

5.20 Pavia Image . . . . . . . . . . . . . . . . . . . . . . . 75

5.21 Results Pavia information classes Signatures. . . . . . . . . . . . . . 76

5.22 Results Pavia information classes abundances. . . . . . . . . . . . . . . . 77

5.23 Comparison of Asphalt and Bitumen classes on Pavia set. . . . . . . . . . . . . 77

5.24 Comparison of Meadows and Bare Soil classes on Pavia set. . . . . . . . . . . . 78

5.25 Comparison of classes on Pavia set. . . . . . . . . . . . . . . . . . . 79

5.26 Pavia Image: (a) Classification map using the abundances and the majority vote criterion; (b) Classification map after filtering; (c) New reference data classification map. . . . . . . . . . . . . . . . . . . . . . . . . 79

5.27 Spectral endmembers extracted from the Urban scene using global analysis . . . 81

5.28 Abundance maps extracted from the Urban scene using global analysis . . . . . 82

5.29 Class map generated using Global approach for Urban scene. . . . . . . . . . . 82

5.30 Spectral endmembers extracted from the urban scene using global analysis on the LD image . . . . . . . . . . . . . . . . . . . . . . . . . . . . . 84 
5.31 Abundance maps extracted from the urban scene using the global analysis on the LD image. . . . . . . . . . . . . . . . . . . . . . . . . . 85

5.32 Class map generated using Global analysis on the LD approach . . . . . . . . . 85

5.33 (a) Quadtree Image Segmentation of Urban scene; (b) Number of endmembers per tile $($ total $=114) . \ldots \ldots \ldots \ldots \ldots \ldots$

5.34 Spectral information classes extracted from the urban scene using the Quad-tree Approach . . . . . . . . . . . . . . . . . . . . . 87

5.35 Abundance maps extracted from the urban scene using the Quadtree approach. . . 88

5.36 Class map generated using the Quadtree approach . . . . . . . . . . . . . 88

6.1 A.P. Hill Hyperspectral image: (a) RGB composition; (b) Reference data [8]. . . . 93

6.2 Spectral library for A.P. Hill. Figure from [8] . . . . . . . . . . . . . . . 93

6.3 New Reference class map after applying the steps discussed in Section 6.3 . . . . . 94

6.4 (a) SLIC segmentation of A.P. Hill image (region size $=16$ ); (b) RGB display of the low dimensional superpixel image. . . . . . . . . . . . . . . . . 96

6.5 Partitioning using quadtree $($ EMs $=$ Endmembers $) \ldots \ldots \ldots$. . . . . . . . 96

6.6 Information Abundance class maps extracted from the A.P. Hill Hyperspectral image using the proposed method . . . . . . . . . . . . . . . . 98

6.7 Information class spectral signatures extracted from the A.P. Hill Hyperspectral image using the proposed method . . . . . . . . . . . . . . . . . . . 99

6.8 Generated class map using the proposed approach . . . . . . . . . . . . 100

6.9 High Resolution image of Fort A.P. Hill . . . . . . . . . . . . . . . . . . . . . 102

6.10 A.P. Hill vegetation maps [9]: (a) Primary Trees; (b) Secondary Trees . . . . . . 103 


\section{LIST OF TABLES}

2.1 Overview of sensor spatial and spectral resolutions . . . . . . . . . . . . 7

2.2 Similarity metrics for hyperspectral data . . . . . . . . . . . . . 31

5.1 Example of a Confusion Matrix . . . . . . . . . . . . . . . . 55

5.2 Meaning of the Value of Kappa [10] . . . . . . . . . . . . . . . . 55

5.3 Relation between spectral endmember classes and information classes for Urban. . 62

5.4 Confusion matrix between reference map and generated classification map from unmixing results. . . . . . . . . . . . . . . . . . . . . . 68

5.5 Confusion matrix between reference map and filtered classification map from unmixing results. . . . . . . . . . . . . . . . . . . . 68

5.6 Relation between Spectral classes and information classes for Pavia scene . . . . 70

5.7 Confusion matrix between reference map and generated classification map from unmixing results. . . . . . . . . . . . . . . . . . . . 80

5.8 Confusion matrix between reference map and filtered classification map from unmixing results. . . . . . . . . . . . . . . . . . . . 80

5.9 Confusion matrix between reference map and generated classification map from global approach. . . . . . . . . . . . . . . . . . 83

5.10 Confusion matrix between reference map and generated classification map from the global LD approach. . . . . . . . . . . . . . . . . . 86

5.11 Confusion matrix between reference map and generated classification map from the quadtree approach. . . . . . . . . . . . . . . . . . 87

5.12 Producer's and User's Accuracies for the 4 methods on the Urban data set . . . . . 89

5.13 Kohen's Kappa and overall accuracies for the 4 methods . . . . . . . . . . . . . . 90

6.1 A.P. Hill class pixel counts and percentages . . . . . . . . . . . . . . . 95

6.2 Confusion matrix between reference map and generated classification map from unmixing results. . . . . . . . . . . . . . . . . . . . . 97

6.3 Confusion Matrix for A.P. Hill Image by $[11] \ldots \ldots$. . . . . . . . . . . . . 101 


\section{Chapter 1 \\ INTRODUCTION}

\subsection{Justification}

Hyperspectral imaging (HSI) systems collect image data simultaneously in hundreds of narrow, adjacent spectral bands. Hyperspectral remote sensing is used in a wide array of applications such as agriculture, mineralogy, surveillance, etc. the high spectral resolution of HSI makes it easier to identify the types of materials in a scene, but processing the data is not trivial since we are dealing with large volume of data that is subject to different sources of variability like atmospheric variability, variation in illumination between pixels among others. Furthermore, in many practical applications, such as threat detection in surveillance systems, it is crucial to perform the processing in real time, making this task even more challenging.

In HSI, the observed radiation represented from a single pixel rarely comes from a pure material. However, the high spectral resolution of the HSI spectrometer enables the detection, identification and classification of subpixel objects and their contribution to the measured spectra. Spectral information collected with hyperspectral remote sensors can be modeled as the convex combination of the spectral signatures of the materials in the sensor field of view. The distinct materials associated with the surface are called endmembers, and the fractions in which they appear in a pixel are called abundances. The unmixing problem refers to finding the number of endmembers, their spectral signatures, and their abundances from a given hyperspectral image.

Application of unmixing to a full HSI scene returns estimates of endmembers and their abundance that model the full spectral data cloud as the convex hull of the extracted endmembers. An important problem is that actual hyperspectral data clouds are not convex but better modelled as mixtures of lower dimensional convex distributions or approximated by piecewise-convex regions. One cause for non-convexity of the data cloud in real hyperspectral imagery is that materials in a scene are not uniformly distributed so certain mixtures of materials are not possible due to spatial constraints. 


\subsection{Problem Statement}

The use of the full image scene to directly extract endmembers might not be accurate since, in real life, the data cloud won't be convex in most cases, non-convexity may occur due to that some materials are not uniformly distributed so certain mixtures are not possible due to spatial constraints. Therefore, it's more appropriate to use local information to extract endmembers from the scene.

The goal of this research is to show how to exploit the relation between the spatial distribution of the materials in a scene and their location in the data cloud, and see how does this spatialspectral interaction can be leveraged for hyperspectral unmixing. We propose to use segmentation techniques to partition the image into uniform regions, we perform endmember extraction on each region. The rationale is that at the local level, it will be easier to capture endmembers with low variance, and the cloud behavior for each region better follow the convex hull model used on unmixing analysis. We then cluster endmembers based on their spectral angle distance to get endmember classes, then unmixing using the endmembers extracted will be performed for the full hyperspectral scene to get the abundance maps.

\subsection{Technical Approach}

This research proposes an approach to perform unsupervised unmixing where endmember classes, composed of multiple spectral signatures, are used to describe the "endmember" across a large scene. Local spectral signatures are extracted from spectrally homogeneous regions. Spectrally homogeneous regions are identified using segmentation techniques like the quadtree method and the Simple linear iterative clustering (SLIC) method. Once the regions are identified, local endmembers can be extracted using any of the available endmember extraction methods. Endmember classes are built by clustering local spectral endmember signatures to build up a more precise description of the landscape under study. The approach is summarized in figure 1.1. 


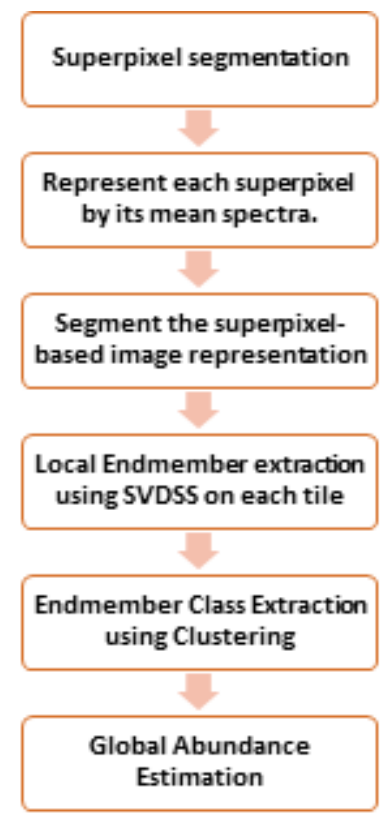

Figure 1.1: Proposed segmentation-based unmixing.

\subsection{Research Objectives}

The main goal of this research is to develop a model that will integrate spatial information into the unmixing problem, the proposed work will automatically segment the hyperspectral image into similar regions, endmember extraction will be performed on each region. Extracted spectral signatures are grouped into spectral endmember classes that represent both the spectral features and the spectral variability of different materials in the image. This approach is implemented in an unsupervised computing system for the analysis and processing of hyperspectral images.

\subsection{Dissertation Outline}

Chapter 2 presents a complete review about the state of the art unmixing analysis of hyperspectral imagery. The linear mixing models is presented in Section 2.3, and the most widely used algorithms for endmember extraction and abundance estimation are reviewed in Section 2.4. Superpixel segmentation using the SLIC approach is discussed in section 2.5. While section 2.6 talks about the segmentation problem in general and in hyperspectral images in particular. In the end, 
section 2.7 talks about the different clustering techniques that are used to group different sets of data based on some similarity criteria.

Exploratory visual analysis to study the relation between spatial and spectral information is shown in Chapter 3. This exploratory analysis serves as the motivation for the structure proposed for this dissertation. We first show how the image pixels are located in feature space in section 3.2. Section 3.3 summarizes how the manual region partitioning will expose the convex regions in the cloud. Section 3.4 shows how the SLIC algorithm is applied as a dimensionality reduction preprocessing step, we showed that even with dimensionality reduction, the cloud still maintained its structure.

Chapter 4 is dedicated to serve as a detailed description of the proposed algorithm.

Chapter 5 presents the results of our approach using hyperspectral images from different scenarios. Section 5.2 describes the data sets employed in these experiments. Section describes the assessment methodology followed in this work. Section shows the unmixing analysis of two hyperspectral images and their validation using classification maps and spectral libraries. Finally, Section 5.5 presents the comparison of the proposed approach with the global and quadtree based unmixing techniques to evaluate the performance of our work.

In chapter 6, we applied our approach on a more complex hyperspectral image, that is the Fort A.P. Hill scene. In section 6.2, we showed the need to formulate a new class map that is based on land covers. Section 6.4 shows the results obtained using our approach, we then evaluate our results with the ones obtained using quadtree approach. Finally, Chapter 7 presents the conclusions on this work as well as indicates possible path for future research. 


\section{Chapter 2}

\section{BACKGROUND AND LITERATURE REVIEW}

\subsection{Introduction}

This chapter presents a review of the current state of the art in hyperspectral image processing emphasizing in the linear unmixing methods that use both spatial and spectral information. In Section 2.1, an overview of hyperspectral imaging is presented. In Section 2.2, the linear mixing model (LMM) is reviewed. In Section 2.3, the spectral unmixing problem is studied, several approaches to determining the number of endmembers, endmember extraction techniques and abundance estimation methods are described. Section 2.4 talks about hyperspectral image segmentation using superpixels, with a focus on the Simple Iterative Linear Clustering (SLIC) algorithm that is used in the research. Finally, Section 2.5 presents a short review about general image segmentation approaches with a focus on the quadtree method.

\subsection{Remote Sensing and Hyperspectral Imaging}

Remote sensing is the technology of getting information for an object without being in actual contact with it. This includes sensing, recording, processing and analyzing that information [6]. Hyperspectral images (HSI) are one type of information that can be acquired using satellites or airborne sensors.it has opened up new possibilities for monitoring earth surface such as minerals that can help identify materials in a scene. Other applications of HSI include surveillance, agriculture [12], food processing [13], and eye care [14]. Figure 2.1 illustrates a typical remote sensing scenario.

Hyperspectral images have this name from the fact that they continuously sample a region of the electromagnetic spectrum at high spectral resolution. Each image is referred as a band. They are different from multispectral images in terms of number of bands, the way spectra is sampled and their spectral resolution. Multispectral images usually have several to tens of bands. Figure 


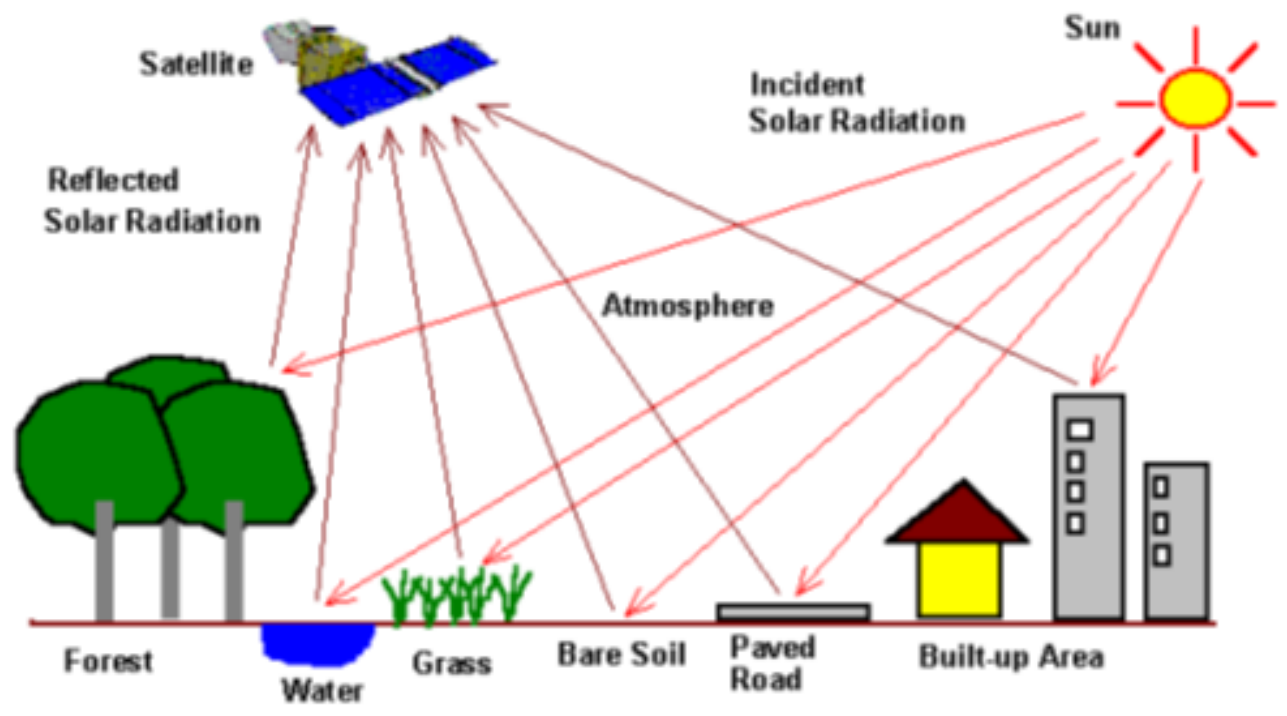

Figure 2.1: overview of Remote sensing system. [1]

2.2 shows a graphical representation of spectral image modulation.

Each image of the HSI corresponds to the same scene, but at different spectral band. Each

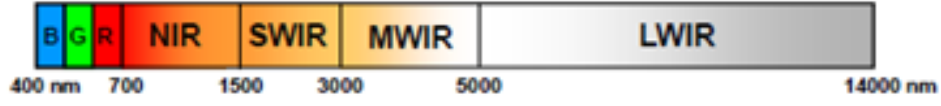

\section{LOW Panchromatic: one very wide band}

\section{MED Multispectral: several to tens of bands}

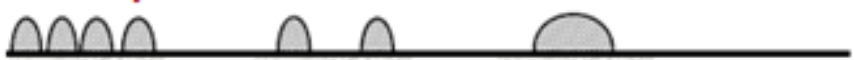

HIGH Hyperspectral: hundreds of narrow bands|

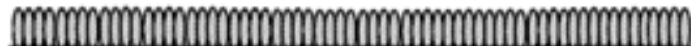

Figure 2.2: Spectral sampling in spectral imaging.

band image (channel) that is captured using the sensor carries certain amount of information. These images contain spatial, spectral, and temporal information about that specific earth surface patch. The finite energy that arise from the earth surface is then spatially divided into pixels. A pixel is the smallest single component in a digital image. Each pixel in each band is associated with a 
Table 2.1: Overview of sensor spatial and spectral resolutions

\begin{tabular}{|l|l|l|l|l|}
\hline Sensor & $\begin{array}{l}\text { Number of } \\
\text { bands }\end{array}$ & $\begin{array}{l}\text { Spatial resolu- } \\
\text { tion }\end{array}$ & $\begin{array}{l}\text { Wavelength } \\
\text { range }\end{array}$ & $\begin{array}{l}\text { Spectral Band- } \\
\text { width }\end{array}$ \\
\hline AVIRIS & 224 & $\begin{array}{l}\text { Depends on the } \\
\text { flight altitude }\end{array}$ & $360-2500 \mathrm{~nm}$ & $9.6 \mathrm{~nm}$ \\
\hline ROSIS & 115 & $\begin{array}{l}\text { Depends on the } \\
\text { flight altitude }\end{array}$ & $430-860 \mathrm{~nm}$ & $4 \mathrm{~nm}$ \\
\hline Hyperion & 220 & $30 \mathrm{~m}$ & $400-2500 \mathrm{~nm}$ & $10-11 \mathrm{~nm}$ \\
\hline HyspIRI & 212 & $60 \mathrm{~m}$ & $380-2500 \mathrm{~nm}$ & $10 \mathrm{~nm}$ \\
\hline
\end{tabular}

number called the digital number or brightness value. For images, that number is related to the average radiance of a relatively small area within a scene.

Remote sensing data obtained from sensors like the Airborne Visible/Infrared Imaging Spectrometer (AVIRIS) [15] are used to identify, measure, and monitor constituents of earth surface and atmosphere. Other airborne sensors like Reflective Optics System Imaging Spectrometer (ROSIS) [16] are used for detection of spectral fine structures especially in coastal and inline waters. AVIRIS has 224 spectral bands with wavelengths ranging from $360 \mathrm{~nm}$ to $2500 \mathrm{~nm}$. ROSIS has 115 spectral bands between $430 \mathrm{~nm}$ to $860 \mathrm{~nm}$.

Satellite hyperspectral sensors, like The Hyperion sensor [17], provides a new class of Earth observation data for improved Earth surface characterization. It provides a high resolution hyperspectral imager capable of resolving 220 spectral bands (from $400 \mathrm{~nm}$ to $2500 \mathrm{~nm}$ ) with a 30 -meter resolution. Another satellite sensor like the Hyperspectral Infrared Imager (HyspIRI) [18] is prepared to study the world's ecosystems and provide critical information on natural disasters such as volcanoes, wildfires, and drought. The HyspIRI will include two instruments, an SWIR imaging spectrometer measuring from: $380 \mathrm{~nm}$ to $2500 \mathrm{~nm}$ in $10 \mathrm{~nm}$ contiguous bands, and a thermal multispectral imager containing 8 bands between 3 to $12 \mathrm{um}$. Both instruments have a spatial resolution of $60 \mathrm{~m}$ at nadir. Table 2.1 shows a comparison between the sensors mentioned above. 


\subsection{Linear Mixing Model}

Hyperspectral images often represent scenes which numerous disparate material substances contribute to the measured spectrum of a single pixel. One of the reasons behind this is that the spatial resolution of the Hyperspectral sensor is larger than the area of the class portion of the image, a pixel would be pure if the opposite happens, but that is not common in HSI sensors.

Many models were introduced to represent how the materials interact to generate the measured spectrum for each pixel. Mixing models attempt to represent the underlying physics that are the foundation of hyperspectral phenomenology. Unmixing algorithms use these models to perform the inverse operation, attempting to recover the endmembers, and their associated fractional abundances from the mixed-pixel spectrum. Figure 2.3 illustrates the two categories of mixing models, the linear mixing model and the nonlinear mixing models.
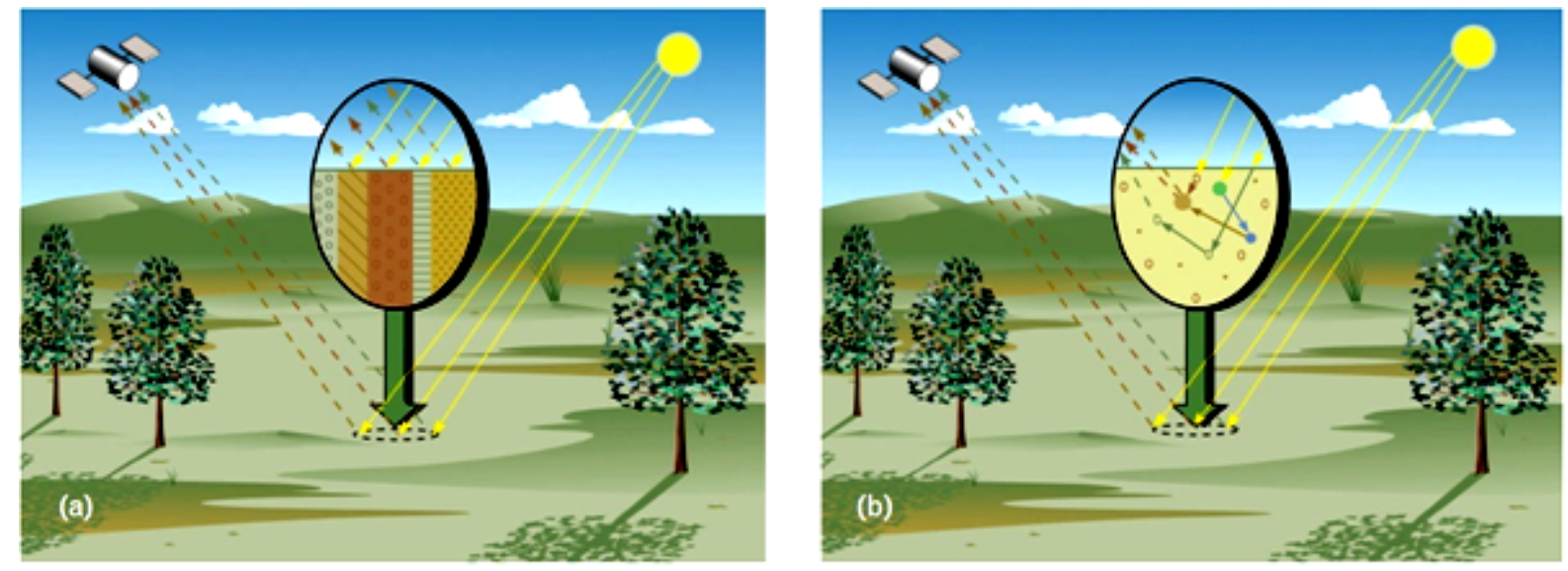

Figure 2.3: Two mixing models. (a) The linear mixing model assumes a well-defined proportional checkerboard mixture of materials, with a single reflection of the illuminating solar radiation. (b) Nonlinear mixing models assume a randomly distributed, homogeneous mixture of materials, with multiple reflections of the illuminating radiation [2]

The linear mixing model (LMM) represents a pixel as the linear combination of the spectral signatures of each material (endmembers) multiplied by its fractional coverage area (or abundance). When $\mathrm{p}$ endmembers exist, each having $\mathrm{m}$ bands, LMM can be expressed as:

$$
\mathbf{x}_{j}=\sum_{i=1}^{p} \mathbf{s}_{i} \mathbf{a}_{j}+\mathbf{w}_{j}=\mathbf{S a}_{j}+\mathbf{w}_{j}, j=1,2,3 \ldots, N
$$


where $\mathbf{x}_{j} \in \mathfrak{R}_{+}^{m}$ is the measured spectral signature at the $j^{t h}$ pixel, $\mathbf{S} \in \mathfrak{R}_{+}^{m \times p}$ is the endmembers matrix, $\mathbf{a}_{j} \in \mathfrak{R}_{+}^{p}$ is the spectral abundances vector, $\mathbf{w}_{j} \in \mathfrak{R}^{m}$ is a measurement noise vector, and $\mathrm{N}$ is the number of pixels.

In HSI, $m>p$, notice that all elements of $\mathbf{S}, \mathbf{a}$, and $\mathbf{x}$ are constrained to be positive, and the sum of $a_{i j}$ for all spectral bands $m$ is less than or equal to one due to the topography of the scene [19]. For the entire HSI, the linear mixing model given above can be written in matrix form as:

$$
\mathbf{X}=\mathbf{S A}+\mathbf{W}
$$

where $\mathbf{X}=\left[\mathbf{x}_{1}, \ldots \mathbf{x}_{N}\right]$ is the matrix containing all image pixels, $\mathbf{S}=\left[\mathbf{s}_{1}, \ldots \mathbf{s}_{p}\right]$ is the matrix of endmembers, $\mathbf{A}=\left[\mathbf{a}_{1}, \ldots \mathbf{a}_{N}\right]$ is the matrix of abundances, $\mathbf{W}=\left[\mathbf{w}_{1}, \ldots \mathbf{w}_{N}\right]$ is the noise matrix and $\mathrm{N}$ is the number of pixels in the image. It's clear that the spatial relations will be lost when thinking of the hyperspectral image as a matrix as described in 2.2.

If the components of interest are in an intimate association, as shown in Figure 2.3b, like sand grains of different composition in a beach deposit, light typically interacts with more than one component as it is multiply scattered, and the mixing systematics between these different components are nonlinear [2].

\subsubsection{Endmembers Spectral Signature}

Spectral Signature is the difference in the reflectance characteristics of materials with respect to wavelengths. Each material has a unique signature, that describes the characteristics of the material as a function of wavelength. For an object, the signature is a function of the incidental Electromagnetic wavelength and material interaction with that section of the electromagnetic spectrum; Figure 2.4 shows the spectral signatures of 3 different materials. It's clear that at $1000 \mathrm{~nm}$ wavelength, the vegetation has the highest reflectance compared to soil and shade. While at the $2000 \mathrm{~nm}$ wavelength, the soil has the highest reflectance and the vegetation has the lowest. This information can be represented in a different way; Figure 2.5 shows the three endmembers in the 


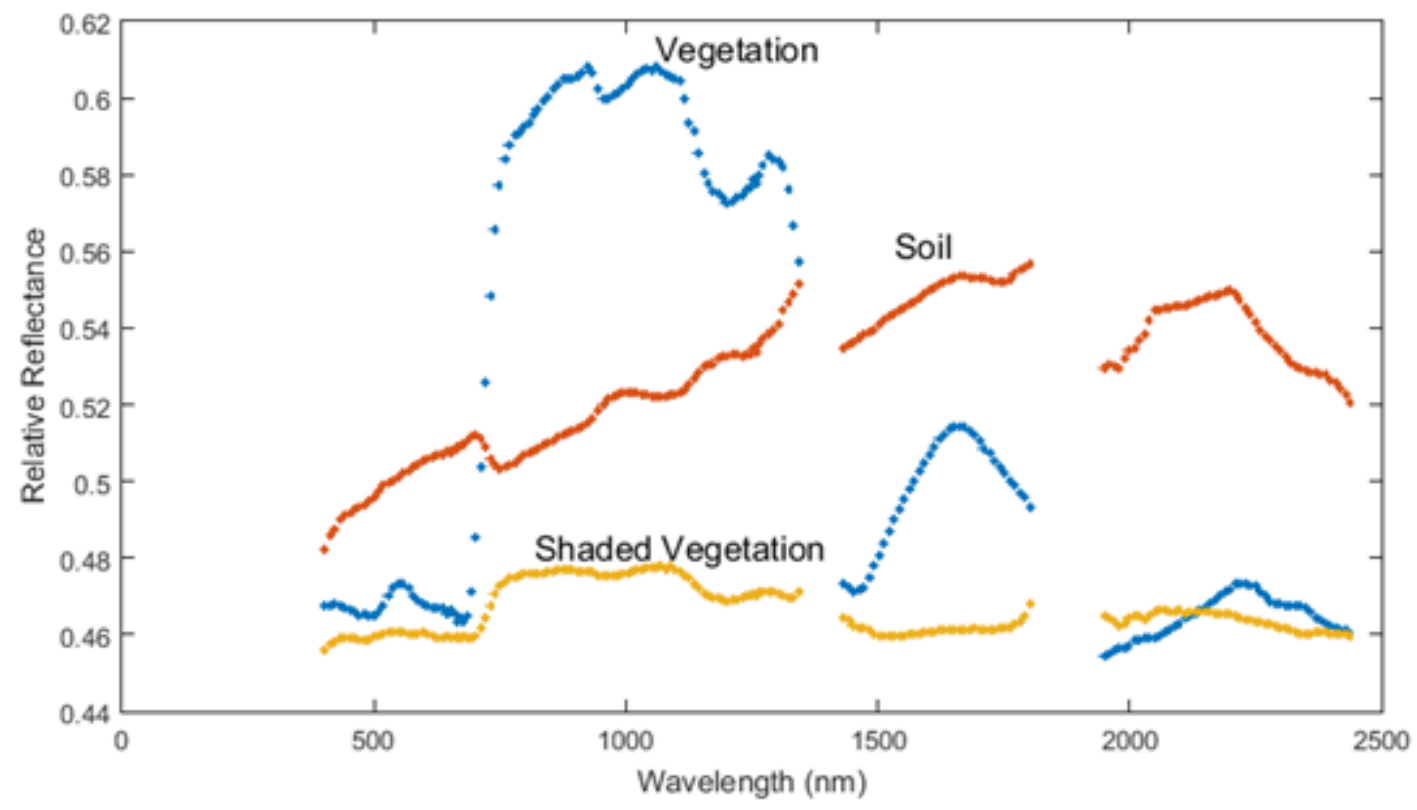

Figure 2.4: Spectral Signatures of 3 different materials.

feature space representation.

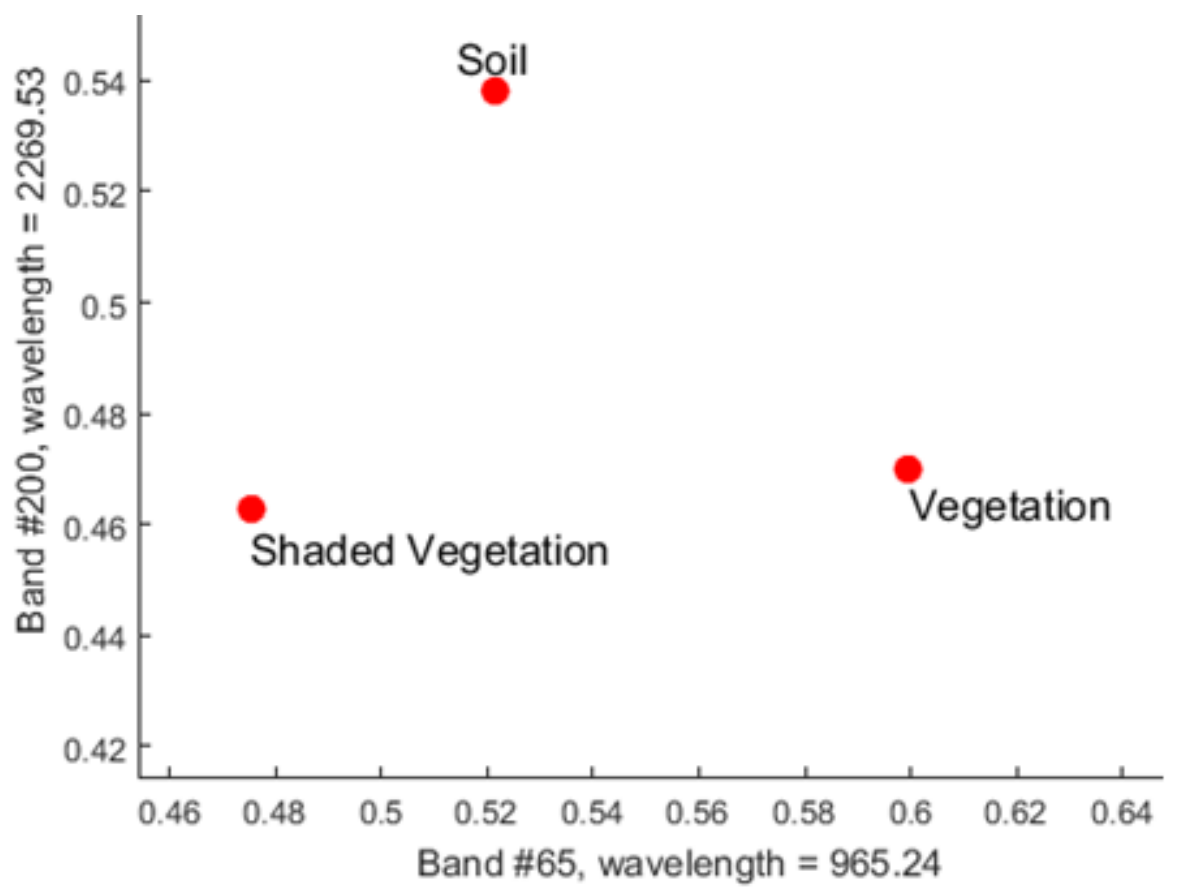

Figure 2.5: Feature space representation of the three pure endmembers.

However, feature space here shows only pure endmembers (no mixing model as in eq. 2.2), case like this doesn't exist in real hyperspectral imagery. Actual representation of image data 
points in feature space is much more like Figure 2.6, where pixels are mixed as in eq. 2.2. This representation is also known as the spectral data cloud.

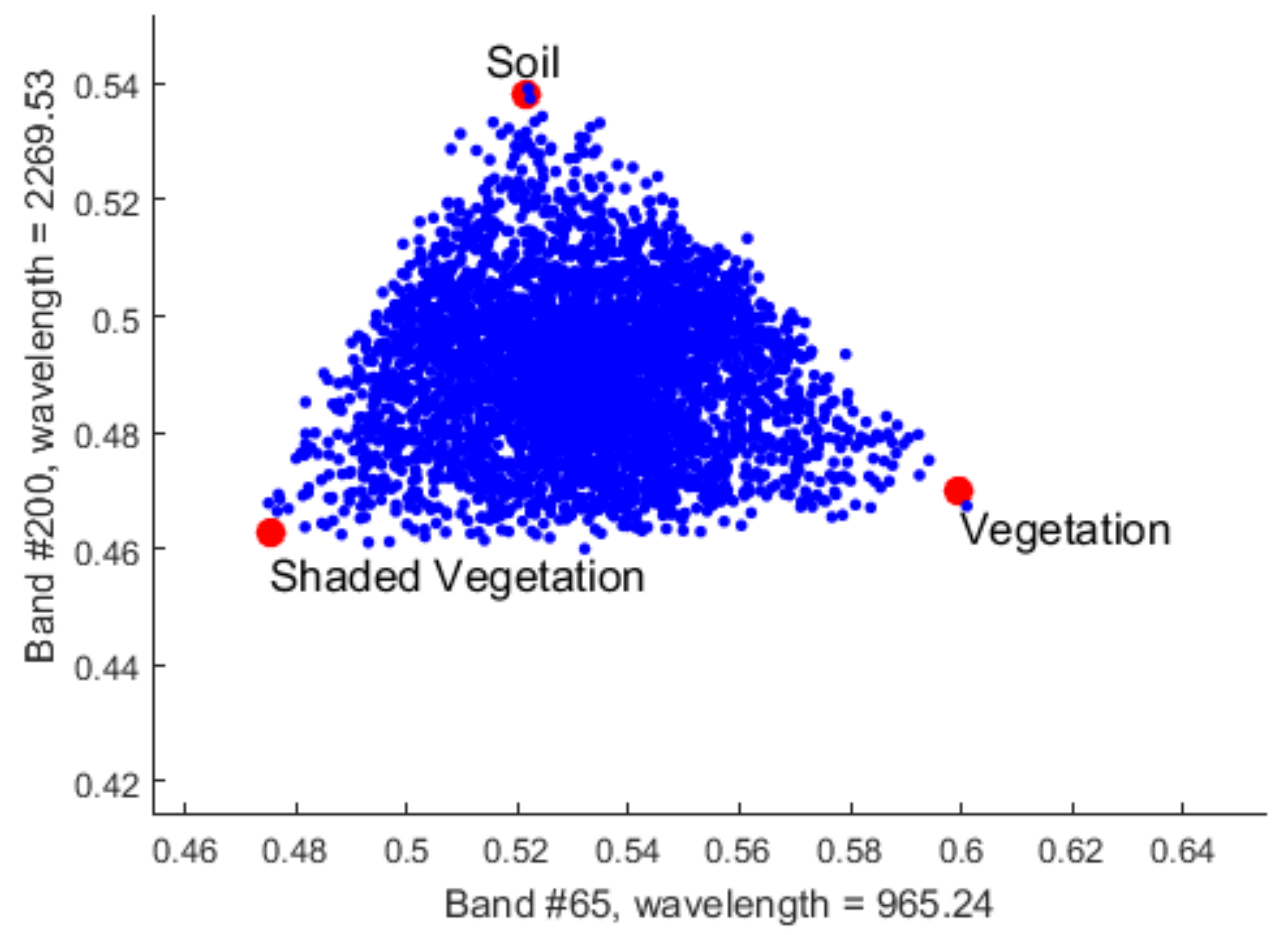

Figure 2.6: Feature space representation of the three mixed endmembers.

\subsection{Spectral Unmixing}

Spectral unmixing is the inverse procedure by which given the image $\mathbf{X}$, we want to determine the number of endmembers $p$, the endmembers matrix $\mathbf{S}$, and the abundance matrix $\mathbf{A}$. Several methods have been proposed to solve the unmixing problem. Many algorithms assume that endmembers are known a priori, so unmixing reduces to abundance estimation. Spectral libraries obtained in laboratories or collected in the field can be used as endmembers. However, in most cases the endmembers are unknown. In this research, the focus will be on unsupervised unmixing algorithms that seek to extract the endmembers signatures from the hyperspectral image itself.

Typical unmixing algorithms can be divided into three steps. The first step consists of determining the number of endmembers in the scene. The determination of the number of endmembers is an important issue and has been studied by many investigators. The second step is the extraction of 
spectral signatures of endmembers. Abundances are then estimated in the third step. Some unmixing algorithms estimate the number of endmembers together with the spectral signature. However, most endmembers extraction algorithms assume that the number of endmembers is known a prior. Determining the number of endmembers in hyperspectral imagery is a challenging task. One cause is that most hyperspectral scenes don't have pure pixels that could be considered as endmembers. Another is that a particular material may not be characterized by a single spectral signature due to spectral variability.

\subsubsection{Endmembers Determination Approaches}

There are several approaches for automatic endmember extraction. The most widely used algorithms are based on the relation between mixing and convex geometry [20][21][22]. In addition, parametric model approaches have been proposed for the endmember extraction problem. Geometric and parametric approaches are reviewed in the next sections. Techniques described in Sections 2.4.1.1 and 2.4.1.2 are per-pixel approaches that do not take into account the spatial information provided by the hyperspectral images. 2.4.1.3 reviews the state of the art of endmember extraction algorithms that use both spatial and spectral information.

\subsubsection{Geometric Methods}

The most widely used algorithms are based on the relation between mixing and convex geometry where the spectral signatures are seen as points in an n-dimensional scatterplot [23]. Due to the non-negativity property of radiance and reflectance, spectral data lie in a convex cone [24]. When thesum to one constraint is added, the spectral data lie in a simplex where the points are linear combinations of the vertices [23]. The vertices or corners of the simplex will be the endmembers. Some examples that are based on the geometrical model are reviewed below:

- Pixel Purity Index (PPI) [25] algorithm projects every spectral vector onto a large set of random unit vectors known as skewers. The points corresponding to extremes, for each skewer direction, are stored. A cumulative account records the number of times each pixel is found to 
be an extreme. The pixels with the highest scores are the purest ones and thus considered as endmembers. This is not a fully automated technique because the final endmembers should be selected by a trained image analyst. PPI assumes that there are pure pixels in the image.

- N-FINDER [26] is based on the fact that in spectral dimensions, the volume defined by a simplex formed by the purest pixels is larger than any other volume defined by any other combination of pixels. This algorithm finds the set of pixels defining the largest volume by inflating a simplex inside the data. First, a set of pixels are randomly selected as endmembers. The volume of the simplex is calculated using the selected pixels as:

$$
V(\mathbf{S})=\frac{a b s(|\mathbf{S}|)}{(p-1) !}
$$

where $p$ is the number of endmembers, $\mathbf{S}$ is the endmembers matrix, abs() is the absolute value and Ildenotes the determinant. The algorithm iteratively replaces every endmember for each pixel in the hyperspectral image calculating the volume. If the volume increases, then the new pixel replaces the candidate endmember. The main problem with this algorithm is that results can be affected by outliers. N-FINDR assumes that there are pure pixels in the image. Also, there is no guarantee that the search will give the pixels with maximum volume.

- Vertex Component Analysis (VCA) [27] is based on the fact that the affine transformation of a simplex is another simplex. The VCA algorithm projects image pixels to a random direction, the pixel with the largest projection is considered the first endmember. The other endmembers are found by projecting the data onto a direction orthonormal to the subspace spanned by the set of endmembers already found, the procedure is repeated until the whole set of endmembers is found. VCA assumes that there are pure pixels in the image.

- $\quad$ Non-negative Matrix Factorization (NMF) was introduced by Lee and Seung [28]. Given a non-negative matrix $\mathbf{Y}, \mathrm{NMF}$ tries to find non-negative matrices $\mathbf{S}$ (Basis matrix) and $\mathbf{A}$ (encoding Matrix), such that $\mathbf{Y} \approx \mathbf{S A}$. For hyperspectral unmixing applications, matrix $\mathbf{S}$ is considered as the endmembers matrix and matrix $\mathbf{A}$ is considered as the abundance matrix, 
while $\mathbf{Y}$ is the matrix of the observed pixels. The advantage NMF over the previous methods is that NMF does not assume that pure pixels are contained in the hyperspectral image. However, the number of endmember should be known a priori. Unmixing can be performed by solving the following optimization problem [29] [30]:

$$
(\overline{\mathbf{S}}, \overline{\mathbf{A}})=\underset{S_{i j} \geq 0, A_{i j} \geq 0, \sum_{i=1}^{p} A_{i} \leq 1}{\operatorname{argmin}}\|\mathbf{Y}\|_{F}^{2}
$$

The main difference of NMF over other methods that it does both endmember and abundance estimation simultaneously.

The methods in [30] describe the use of a constrained positive matrix factorization for unsupervised unmixing. in [5], quad-tree partitioning is used to divide the data into spectrally homogeneous regions and then performs NMF to extract local endmembers on each tile, the extracted endmembers are then clustered to form spectral classes. This adds spatial context to NMF based unmixing.

\subsubsection{Parametric Methods}

Parametric approach models endmember and fractional abundance as random vectors. Most parametric algorithms assume Gaussian distributions for the pure constituents [2]. Several parametric models can be found in the literature such as stochastic mixing model [31], dependent component analysis [32], endmember detection using the Dirichlet process [33], piece-wise convex endmember detection (PCE)[20], and reversible jump Markov chain Monte Carlo algorithm [34].

\subsubsection{Spatial-Spectral Methods}

Algorithms described above only take into account the spectral information of hyperspectral imagery with no regards to the spatial distribution of the endmembers in the scene. Some endmembers occupy small areas in the image and will be hard to detect using classical endmember extraction approaches. Spatial information has been incorporated in the hyperspectral analysis us- 
ing well-known image processing techniques such as morphological analysis, spatial kernels, and segmentation. Some spatial-spectral approaches are summarized below:

- $\quad$ Automated Morphological Endmember Extraction (AMEE) is a fully automated endmember extraction algorithm proposed by [3] based on mathematical morphology operations extended to multispectral images. AMEE estimates the number of endmembers and looks for them using spatial kernels. Input parameters to the algorithm are the minimum and maximum size of the spatial kernel. The kernel $\mathrm{K}$, initially with the minimum size, is moved through all image defining local neighborhoods around each pixel.

Extension of morphological operators (i.e. dilation and erosion) to multispectral and hyperspectral imagery are used to define a vector ordering relation. AMEE describes an ordering method based on the spectral purity of a pixel. The spectrally purest pixels $\mathbf{p}$ are related to the dilation operation and spectrally mixed pixels $\mathbf{m}$ are related to the erosion operation. Dilation and erosion are defined for a pixel $\mathrm{y}(\mathrm{x}, \mathrm{y})$ and a kernel $\mathrm{K}$ as:

$$
\begin{aligned}
& p=\operatorname{argmax}\{\mathrm{D}(\mathrm{y}(\mathrm{x}+\mathrm{s}, \mathrm{y}+\mathrm{t}), \mathrm{K})\} \\
& (s, t) \in K \\
& m=\operatorname{argmin}\{\underset{(s, t) \in K}{\{\mathrm{D}(\mathbf{y}(\mathrm{x}-\mathrm{s}, \mathrm{y}-\mathrm{t}), \mathrm{K})\}}
\end{aligned}
$$

where $\mathrm{D}$ denotes a distance metric given by:

$$
D(\mathbf{h}(x, y), k)=\sum_{s} \sum_{t} \operatorname{dist}(\mathbf{h}(x, y), \mathbf{h}(s, t)) \quad \forall(s, t) \in K
$$

and dist() is the spectral angle distance. The effects of dilation and erosion in spectral data are illustrated in Figure 2.7. Dilatation expands regions with pure pixels while erosion shrinks zones with pure pixels.

A morphological eccentricity index (MEI) is obtained by calculating the spectral angle distance between $\mathrm{p}$ and $\mathrm{m}$. MEI is assigned to the maximum pixel in the neighborhood. This 


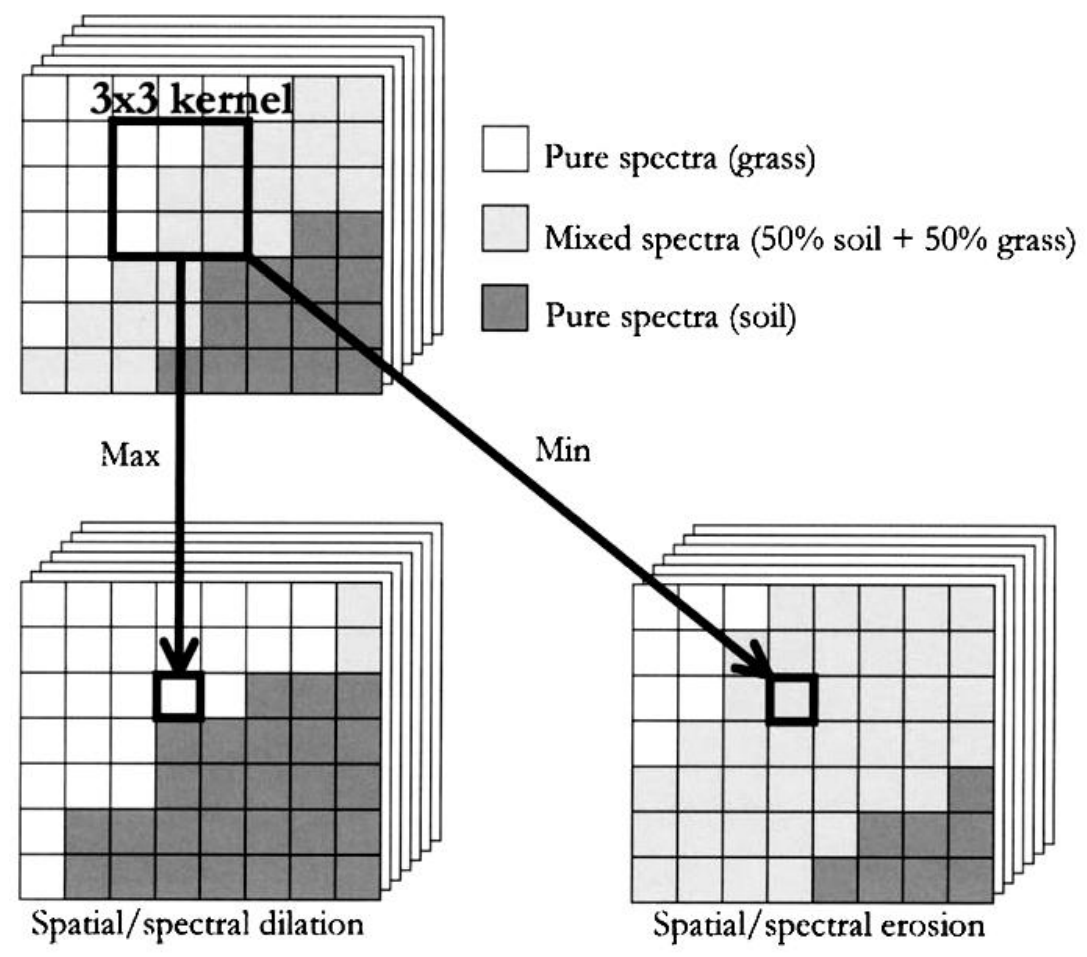

Figure 2.7: Local and global effects of applying spatial-spectral dilation and erosion operations to common hyperspectral image [3]

index determines the capacity of a pixel to describe other pixels in the kernel. The process is repeated for all pixels in the image using kernels of increasing size until the maximum spatial kernel size is reached. The MEI value is updated at each one of the iterations. A threshold is applied to the MEI for unsupervised endmember selection. The number of endmembers correspond to the number of pixels that are not eliminated from the MEI. A region growing procedure is used to refine the final selection. Kernels allow to define local neighborhoods in the search of spectral purity pixels. Thus, AMEE takes into account the spatial and spectral information of the hyperspectral data for endmember extraction. Dilation and erosion operation are extended to determine maximum and minimum spectra in term of the spectral purity of pixels. The MEI index defined in [3]. It allows the selection of those pixels with high spectral purity (determined by the dilation operation) and few mixed (determined by the erosion operation).

- Spatial-Spectral Endmember Extraction (SSEE) algorithm [35] is based on the fact that end- 
members with high spectral contrast are easy to detect using any convex technique. On the other hand, the detection of endmembers with low spectral contrast with respect to the full image is a more difficult task. SSEE breaks up the full image into several subsets increasing the relative contrast of endmembers. SSEE consists of four steps. First, the largest eigenvectors from the SVD of each subset are selected. This set of eigenvectors explains most of the spectral variability of the image. Second, the full image is projected onto the selected eigenvectors. Pixels lying at the extreme are candidates for endmembers. Third, spectrally similar candidates are averaged if they are spatially related. In the last step, endmembers are ordered into a list by using the spectral angle such that the user can identify spectral endmember classes. First endmember is selected as the first spectra in the ordered list. Spectral endmember classes are determined manually by the user identifying groups of similar spectra in the ordered list.

Three parameters are required for SSEE: the pixel subset size, the threshold to select the largest eigenvectors, and the threshold to average similar endmembers in the third step. [35] suggest a subset size of 20 pixels, the first threshold is fixed such that the selected eigenvectors represent the $99 \%$ of spectral variability, and the second threshold is suggested to be equal to one degree if the spectral angle distance is used.

- $\quad$ the Spatially Adaptive Hyperspectral Unmixing [36] algorithm uniformly divides the image into several tiles that are used to identify local endmembers. The endmember extraction in each tile is performed using per-pixel techniques. In [36], MAX-D [37] is used for the determination of local endmembers. MAX-D requires the number of endmembers as input. The Gram matrix [36] is used to estimate the number of endmembers. Abundances are estimated in each tile using local endmembers and similar endmembers are clustered to determine global abundances. As the abundances are estimated locally, a tiling artifact is observed in the final abundances.

- The Spectral Endmember Extraction based on Multiscale Representation Approach, [38] takes advantage of the sampling operation used to build the coarse grids in a multigrid nonlinear 
diffusion method [4]. The endmember extraction method exploits the multigrid structure at the selected scale to determine the spectral endmembers. This technique extracts spectral signatures and spectral endmember classes from hyperspectral imagery in an unsupervised fashion. The spatial-spectral unmixing approach uses the multiscale representation to determine locally spectrally uniform regions and their representative spectra avoiding the explicit segmentation of the hyperspectral image.

A multiscale representation of the hyperspectral images is obtained using nonlinear diffusion. Then, spectral endmembers are automatically identified using multigrid methods to solve the diffusion partial differential equation. The multiscale representation and multigrids allow to avoid the use of spatial kernels. When the spectral endmembers are found, similar spectra are clustered to assemble spectral endmember classes, hence accounting for the spectral variability of the materials in the unmixing analysis, see Figure 2.8

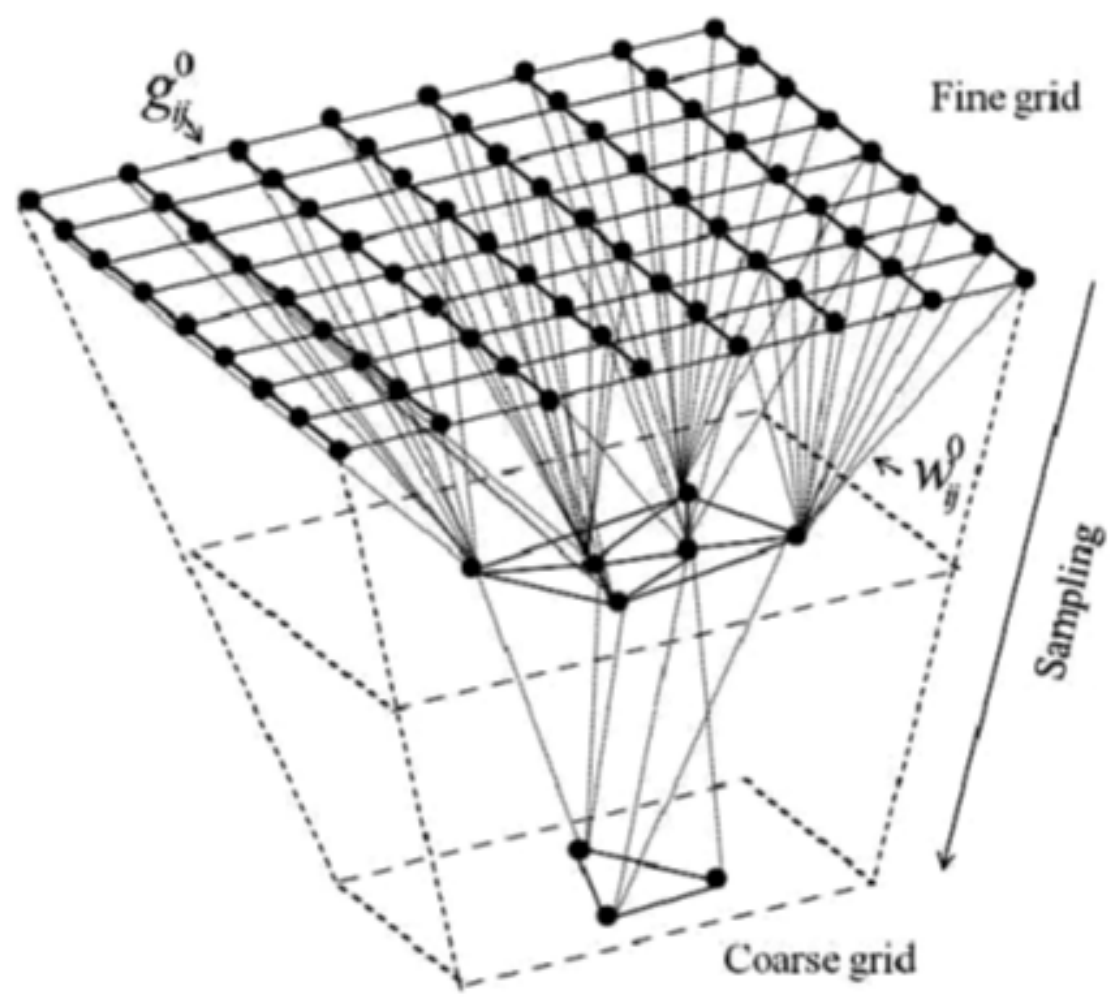

Figure 2.8: Multigrid structure [4]

In the coarsest grid, the equation is solved exactly and then, the solution is propagated back 
to the finer grid. Each grid is represented by a graph. The vertices in the initial graph are the pixels in the original hyperspectral image and the initial edges are built using the closest neighbors. A sampling operation is responsible for building the next grid by selecting those vertices that represent a large number of vertices in the previous one. These vertices are selected such that they represent the vertices in the previous grids. Vertices in the coarsest grid represent the main spectral features of the image. The vertices in the coarsest grid (Figure 2.8 ) are used as the spectral endmembers. Most of the existing spatial-spectral endmember extraction techniques require the use of a spatial window. In contrast, this approach takes advantage of the properties of partial differential equations and multigrid methods to select the spectral endmember without the need to define neighborhoods. This method only uses one of the smoothed images from the multiscale representation for spectral endmember extraction [38].

- $\quad$ The Spatially Adaptive Constrained Non Negative Matrix Factorization for Hyper Spectral Unmixing [29] starts with dividing the image into spectrally homogeneous tiles using the quad-tree partitioning method based on the Shannon entropy [39]. The quadtree splits the image into 4 quadrants; if the entropy of each quadrant is larger than the full image entropy, then the quadrant will be divided into 4 quadrants, this method keeps going until some certain stopping criteria is fulfilled or minimum tile size is reached. Figure 2.9 shows an example of quadtree segmentation on a hyper spectral image.

Endmember extraction will be performed on each tile using the non-negative Matrix Factorization [30] [5]. This step will help capture endmembers at the local level. The extracted endmembers are clustered using the angle distance to form endmember classes[5].

\subsubsection{Determining the Number of Endmembers}

Determining the number of endmembers is the first step and very essential for the unmixing process. Most endmember extraction methods consider the number of endmembers as an input to the algorithm, for this reason the estimation of the number of endmembers also has to be considered 


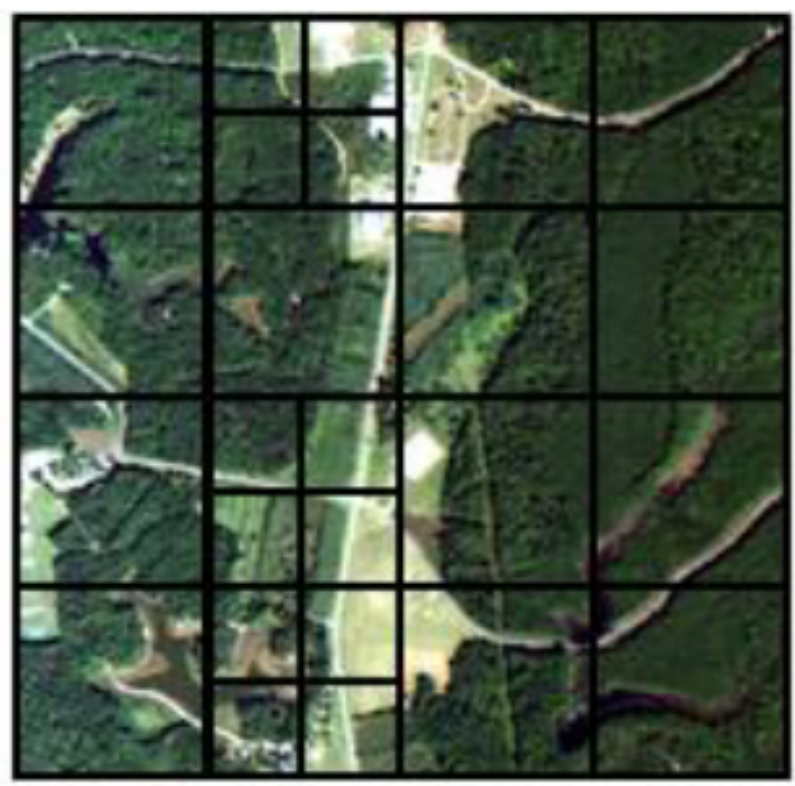

Figure 2.9: Image Partitioning using quadtree [5]

in the analysis of the unmixing problem solution.

Estimators based on the signal subspace rank assume that the number of endmembers is equal to the rank of the covariance matrix. The sensed data $\mathrm{Y}$ is the result of the sum of the original signal $\mathrm{X}$ and noise $\mathrm{W}, Y=X+W$. Then, the covariance matrix of the sensed data $K_{Y}$ is equal to the sum of the covariance matrix of the signal $K_{X}$ and the covariance of the noise $K_{W}$. An estimator based on the signal subspace rank seeks to estimate the rank of the covariance matrix $K_{X}$. Most estimators that use this approach assume that the noise is an independent and identically distributed random vector with $W=\sigma I$.

Principal Component Analysis (PCA) [40] is a linear process that uses an orthogonal transformation to convert a set of observations of possibly correlated variables into a set of values of linearly uncorrelated variables called principal components. The number of endmembers is estimated to be the number of principal components that describes $95 \%$ or $99 \%$ of the entire data.

Rank estimators based on information criteria such as minimum descriptor length (MDL) or Akaike information theoretic criteria (AIC) are part of this category. These approaches have been used for the estimation of the number of endmembers [41], [34]. AIC and MDL were developed in [42] and [43] respectively, and extended for signals embedded in white noise by Wax and Kailath [44]. 
The main assumptions of these model selection methods are that the observations $Y_{i}$ and the noise $W$ are identical and statistically independent with Gaussian distributions and zero mean.

[45] shows that both AIC and MDL do not estimate correctly the number of endmembers since the noise of hyperspectral images is not statistically independent.

Other approaches assume that the number of endmembers is equal to the rank of the image matrix $\mathrm{Y}$ that can be estimated using the correlation matrix given by:

$$
R_{X}=\frac{1}{N} X^{T} X
$$

where $\mathrm{N}$ is the number of pixels in the image. Note that $\operatorname{rank}(\mathrm{X})=\operatorname{rank}\left(R_{X}\right)$

A widely used procedure to compute the number of endmembers is the virtual dimensionality (VD) proposed by Chang and Du [41]. VD compares the eigenvalues from the covariance matrix and correlation matrix to estimate the number of endmembers. However, Bajorski [46] shows that VD is based on incorrect assumptions and won't produce correct results.

The positive rank of the image matrix $\mathrm{Y}$ can be also used in the estimator for the number of endmembers [30]. The positive rank is defined as the least integer $\mathrm{p}$ for which a positive matrix factorization exists [47], i.e.:

$$
\mathbf{Y}=\mathbf{S A}
$$

where $\mathbf{S} \in \mathfrak{R}_{+}^{n \times p}$ and $\mathbf{A} \in \mathfrak{R}_{+}^{p \times m}$.

Bioucas-Dias and Nascimiento propose in [48] the hyperspectral signal identification by minimum error (HySIME) algorithm for the estimation of the dimensionality of a hyperspectral image. First, HySIME estimates the signal and the noise correlation matrices using multiple regression theory. The signal subspace is determined by selecting a subset of eigenvector of the signal correlation matrix. This subset is determined by minimizing the mean square error between image and a noise projection.

The gram matrix method used by [49][50] is a simple and fast method to find the number of endmembers in a scene, the gram matrix is a square matrix where the $\{i, j\}$ th element is the inner 
product between $i_{t h}$ and $j_{t h}$ vectors. One unique property of the Gram Matrix is that the determinant, the Gramian, is the square of the volume of the parallelepiped formed by the vectors [51]. Here the Gramian is computed for the set of endmembers starting with three (to form the convex hull) and increase the number of endmembers until the Gramian approaches zero. The endmembers are ordered by decreasing magnitude such that the convex hull volume generally reaches a peak at a small number of endmembers and is then a monotonically decreasing function.

\subsubsection{Abundance Estimation}

When the endmember signatures are estimated, the next step is to estimate the abundances for each endmember. Abundance estimation is usually performed with constrained least square methods. However, methods using sparse regression have been proposed recently. Techniques based in sparse signal representation theory are used to find the more appropriated endmembers to model each pixel when multiple spectral signatures per endmember are used. This section reviews both approaches.

\subsubsection{Least Square Method}

Given a spectral vector $\mathrm{x}$ and the endmember matrix $\mathbf{S}$, the abundance vector can be estimated by solving the least square problem:

$$
\bar{a}_{j}=\underset{a_{i j} \geq 0, \sum_{i=1}^{p} a_{i}=1}{\operatorname{argmin}}\left\|x_{j}-\mathbf{S} a_{j}\right\|_{2}^{2}
$$

The abundance represents the percentage area covered by a given endmember $\mathbf{S}_{i}$ in a pixel. Therefore, abundances are subject to non-negativity and full additive constraints as shown in eq. 2.10 . The second constraint can be relaxed to sum less than or equal to one to take into account shadow endmembers or effects of topography [19] [52]:

$$
\sum_{i=1}^{p} a_{i} \leq 1
$$


Several solution methods have been proposed in the literature for Abundance Estimation Problem. The simplest solution presented in the literature is to completely ignore the constraints which lead to an unconstrained linear least squares problem with direct solution given by:

$$
\bar{a}_{U L S}=\left(\mathbf{S}^{T} \mathbf{S}\right)^{-1} \mathbf{S}^{T} \mathbf{X}
$$

The simplest constraint to enforce is the sum to one constraint since a direct solution still exists for this case and is given by:

$$
\bar{a}_{S T O}=\bar{a}_{U L S}+\left(\mathbf{S}^{T} \mathbf{S}\right)^{-1} \lambda 1_{p}
$$

where

$$
\lambda=\frac{1-1_{p}^{T} \bar{a}_{U L S}}{1_{p}^{T}\left(\mathbf{S}^{T} \mathbf{S}\right)^{-1} 1_{p}}
$$

This is also simple to implement but still has the same disadvantage as unconstrained linear least squares in that negative abundances could be obtained.

\subsubsection{Library Based Unmixing}

Recently, sparse representations have attracted the attention of the remote sensing community. Several applications of sparse representation for hyperspectral imagery can be found in the literature $[53][54][55]$.

Iordache et al. [55] present a study of sparse unmixing using a spectral library and endmembers extracted from the hyperspectral image using VCA and N-FINDR. When pixels in a hyperspectral image are expressed as a linear combination of a subset of endmembers from a very large spectral library $\mathbf{S}$, the unmixing problem can be reformulated as the problem of seeking the optimal subset of spectral signatures that best model each mixed pixel. Under this assumption, the abundance vector becomes a sparse vector. The sparse unmixing problem can be expressed as:

$$
\begin{array}{ll}
\underset{a}{\operatorname{minimize}} & \|\mathbf{a}\|_{0} \\
\text { subject to: } & \|\mathbf{y}-\mathbf{S a}\|_{2} \leq \boldsymbol{\delta}, a_{i} \geq 0, \sum a_{i} \leq 1
\end{array}
$$


where $\|\quad\|_{0}$ is the zero-norm and denotes the number of nonzero elements, and $\delta$ is a fitting error threshold.

\subsection{Superpixel Segmentation}

Superpixel segmentation is a segmentation where the total number of segments is larger than the number of objects in the image, which makes it an over-segmentation of the image. The number of segments depends on the superpixel size. Superpixel segmentation groups similar pixels in local image spatial neighborhood. If a pixel and its neighbors are similar, then they can be treated as a single entity in subsequent image processing tasks. The computational requirements are significantly reduced since the number of superpixels in an image may be significantly lower than the number of pixels in the uniform grid.

Most of image processing techniques are pixel-based and may be computationally expensive depending on the dimensions of the image. Reducing the image dimension may help accelerating the processing speed while getting similar (or sometimes better) results when compared to the pixel-based methods. Dimensionality reduction algorithms have been used in a variety of imaging analysis applications to provide low-dimensional representations of the image data. However, it is important that dimensionality reduction algorithms yield representations that preserve the structure of the image.

Superpixels incorporate spatial connectivity to group similar pixels in a spatial neighborhood. If a pixel and its neighbors are similar, they can be treated as a single entity in subsequent processing. Therefore, superpixels can result in reduced computational requirements for the image processing task, since processing is performed in the reduced set of superpixels and results are interpolated to the original image pixels. A lower dimensional representation of the image can be obtained, for example, by representing each superpixel by its mean spectral signature. This averaging has a denoising effect, which is another important advantage of superpixel representation. Superpixel-based representations have shown to be successful in improving the performance of hyperspectral unmixing and noise reduction [56] [57]. 
The value of the superpixel representation in image processing and computer vision has been recognized in the literature. Simple linear iterative clustering (SLIC) [58], region based [59], mean shift [60] and water shed [61] approaches are popular techniques for superpixel segmentation. The SLIC algorithm showed better performance in superpixel segmentation than other methods in terms of computation speed and memory efficiency [58]

\subsubsection{Simple linear iterative clustering}

The Simple Linear Iterative Clustering (SLIC) is a simple method to decompose an image into homogenous regions. It's based on a spatially localized version of the k-means clustering.

SLIC starts by dividing the image into an equally spaced grid. The center of each grid tile is then used to initialize a corresponding k-means clustering. After k-means, SLIC optionally removes any segment whose area is smaller than a predefined threshold by merging them into larger superpixels. The step by step process goes as follows:

1 -Divide the image domain into a regular grid with $M \times N$ tiles, where:

$$
M=\left\lceil\frac{\text { ImageWidth }}{\text { RegionSize }}\right\rceil, \quad N=\left\lceil\frac{\text { ImageHeight }}{\text { RegionSize }}\right\rceil
$$

where the region size is a predefined parameter set by the user.

2 - The center of each tile will be used as the initial point (seed point) of a k-means clustering algorithm.

3 -After k-means has converged, SLIC eliminates any region whose area is less than a predefined minimum size. This is done by merging regions to adjacent larger ones. The minimum size for the region to be considered large equals to $\frac{1}{6}$ (RegionSize $)^{2}$ [62].

Figure 2.10 shows the result of SLIC segmentation with different region sizes on a chip from the AVIRIS A.P. Hill scene. 


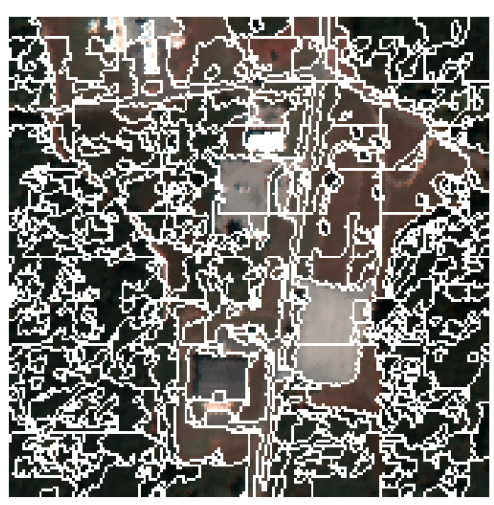

(a) Region Size $=16$

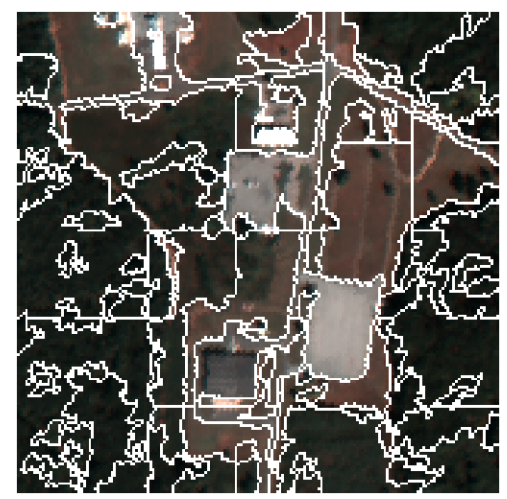

(b) Region Size $=32$

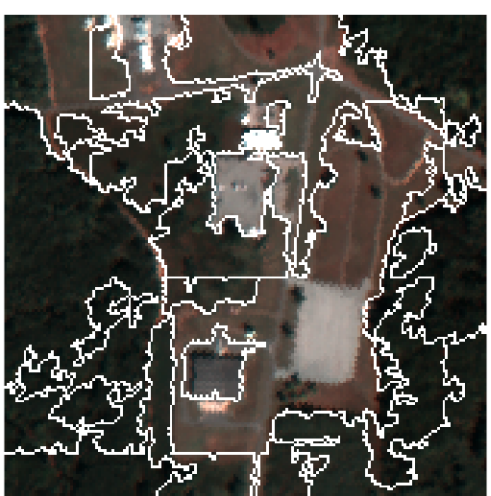

(c) Region Size $=64$

Figure 2.10: Superpixel segmentation for a chip from A.P. Hill Scene

\subsection{Hyperspectral Image Segmentation}

Segmentation problems can be divided into two parts, disjunctive and conjunctive approaches. Disjunctive approach starts with the full image (basic segmentation) then start dividing the image until a uniformity criterion is fulfilled. While the conjunctive approach begins with each pixel and starts to region growing based on a uniformity criterion. The process is repeated iteratively until all pixels of the image are tested [6].

\subsubsection{Disjunctive Approach}

The Disjunctive methods starts with a very simple partition (the entire image) then split the divisions until each element fulfills the wanted numerical uniformity criterion (see Figure 2.11)

In this approach, the scene is divided iteratively and for each piece a global statistical homogeneous index is calculated and compared with the index of the entire scene. If the values are less than the global, partitions are stopped. If the value is larger than the global, this tile is divided in four parts until the homogeneous regions are found in the image [6].

\subsubsection{Conjunctive Approach}

The conjunctive method begins using a very little division of the image and join contiguous components together to form uniform objects. In this approach, object seeking involve cell selection 


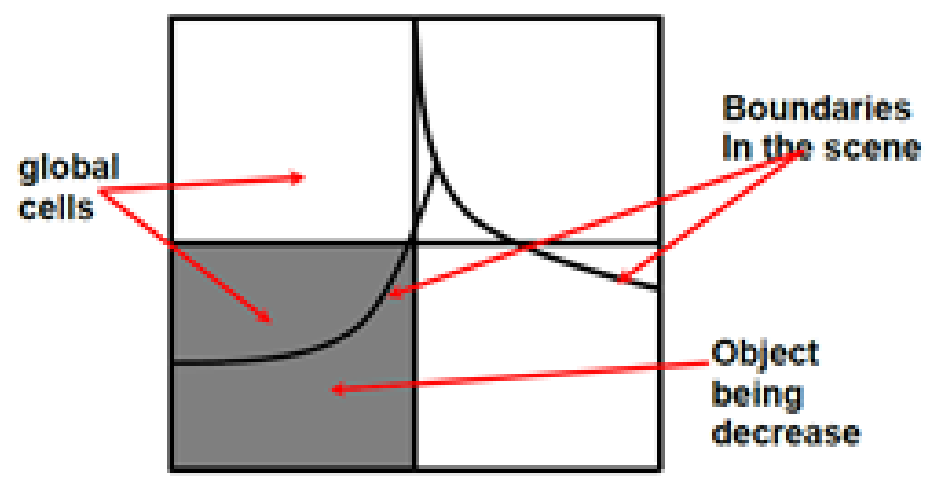

Figure 2.11: Example of Disjunctive Approach [6]

and cells merging (see Figure 2.12) [6].

This method consists of testing a cell with a minor check of numerical uniformity. If it conforms,

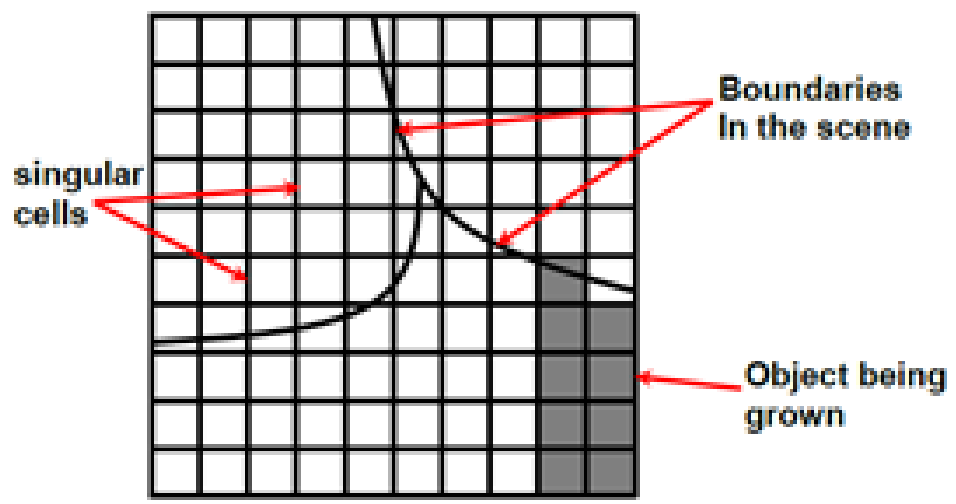

Figure 2.12: Example of Conjunctive Approach [6]

check the neighbor on the left, if it conforms also use a numerical check to decide if the two neighbors have similar statistics. Thus then, the second cell is merged with the first [6]. The process is repeated iteratively until the test fails showing an edge in the image and then a homogeneous region (object) is created. This segmentation process would be supervised or unsupervised, wherein information of the classes would be used. 


\subsubsection{Quadtree Partitioning}

In quadtree segmentation, the image is divided into tiles or quadrants according to some homogeneity criteria. In hyperspectral images, many homogeneity criteria were studied [63] to see which performs a better descriptive segmentation, it is later shown that the Shannon entropy performs better than other metrics as shown in [63]. The Shannon entropy for a region $\mathrm{R}$ is given by:

$$
\operatorname{Shannon}(\mathbf{R})=-\sum_{i=1}^{T} p_{i} \log p_{i}
$$

where $\mathrm{T}$ is the number of clusters in $\mathrm{R}$, and pi is the ratio between the number of pixels in cluster $\mathrm{i}$ to the total number of pixels in $\mathrm{R}$.

Figure 2.13 illustrates the basic idea of quadtree segmentation. The image is first divided into 4 quadrants and the Shannon entropy is computed for each quadrant. If the entropy for the resulting tile is higher than $90 \%$ of the global entropy, the tile is divided again into 4 tiles. The process is repeated until the resulting tile have an entropy lower than $90 \%$ of the global entropy or the tile is of size $8 \times 8$. Note that the leaves of the resulting tree correspond to spectrally homogeneous regions.
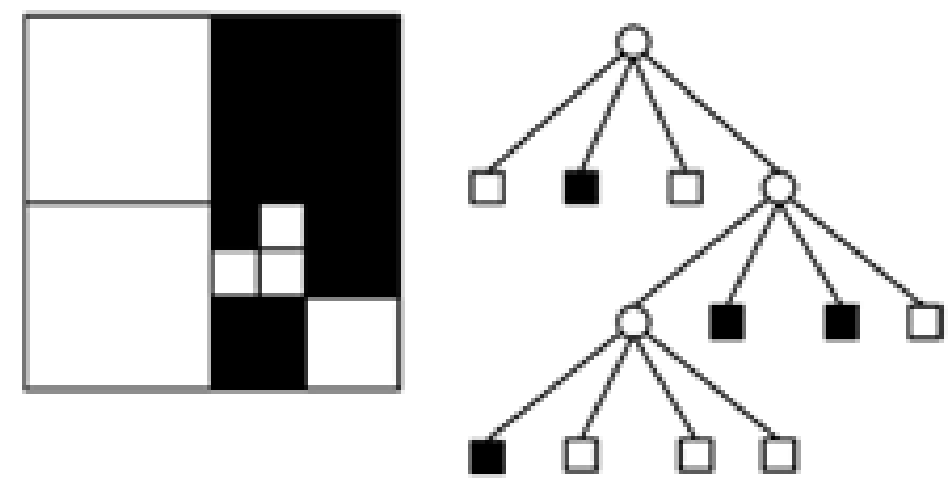

Figure 2.13: Quadtree image representation 


\subsubsection{Segmentation HSI Using SLIC}

Dimensionality reduction is commonly applied to hyperspectral image data as a preprocessing step in algorithms for clustering, segmentation, classification, target detection, and anomaly detection. Nonlinear graph-based dimensionality reduction algorithms such as Laplacian Eigenmaps (LE) [64] and Schroedinger Eigenmaps (SE) [65] have been shown to be very effective at yielding lowdimensional representations that reflect the structure of the manifolds in high-dimensional space on which the original data reside. However, the steps of graph construction and eigenvector computation required by LE and SE can be very costly as the number of hyperspectral image pixels increases. To solve this issue, [62] proposed pre-clustering the hyperspectral image into SLIC superpixels. Each superpixel may represent spatially adjacent image pixels that share common features. Performing LE- or SE-based dimensionality reduction with the superpixels as input significantly reduces the computational effort required both for graph construction and for eigenvalue computation.

\subsection{Clustering Algorithms}

A large number of spectral endmembers can be extracted with the proposed approach. These spectral endmembers represent the distinct components in a scene as well as their spectral variability. Thus, it is necessary to group spectral endmembers into sets with similar spectral features, i.e. meaningful clusters that represent the distinct materials in the image. These meaningful sets of spectral endmembers are the spectral endmember classes.

The obvious way to build the spectral endmember classes is by using clustering techniques. Clustering is widely used in the fields of data mining, statistical analysis, machine learning, pattern recognition, and others. Clustering has been also used in hyperspectral imaging for applications in segmentation, classification, change detection, and unmixing (e.g [36], [66], [67], [68], [69]. [70]). Most of previous works have used clustering over the full image. In this work, cluster analysis is conducted in a small subset of spectra: the spectral endmembers. Thus, the computational cost is 
far less than in the other applications. Some unmixing methods have employed clustering to form sets of endmembers such as [36] and [66].

In this chapter, a brief review of clustering techniques is presented. One of the most challenging problems in clustering analysis is the determination of the number of clusters that in the proposed approach corresponds to the number of spectral endmember classes.

Clustering helps us to divides a collection of endmembers into several subsets (clusters), based on some similarity criterion [71] [72] [73], and the partition clustering seeks a n-partition, $\boldsymbol{C}=\left\{C_{1}\right.$, $\left.C_{2}, \ldots, C_{n}\right\}$, of a dataset $S=\left\{S_{1}, S_{2}, \ldots, S_{m}\right\}$, with $n \leq m$, such that each sample of S belongs to one single partition $C_{i}$.

Clustering methods can be hierarchical, partitional or based in graph theory. A large number of these algorithms perform clustering using a pair-wise distance matrix instead of the dataset and a satisfactory selection of the similarity metric is necessary [71].

Cluster analysis consist of several steps [72]. First, feature extraction techniques can be used to improve the representation of the data. Second, a similarity matrix is calculated using similarity measures. The clustering algorithm performs the separation of the samples into useful subsets. Finally, clustering results are assessed to determine how well the algorithm finds the hidden data structure. This can be done using validity indexes. This section presents a brief summary of what is necessary to perform these steps.

Similarity metrics are used to compare two spectral signatures and establishing how close they are, The more used metrics are the Euclidian Distance (ED), the Spectral Angle measure (SAM) [74], the Spectral Correlation measure (SCM) [75], and the Spectral Information Divergence (SID) [76]. Table 2.2 summarizes these four similarity measures for two spectral signatures A and B. Clustering algorithm techniques, can be classified as hierarchical, partitional and graph theory approaches.

The hierarchical approach makes a group of nested partitions that can be represented by a dendrogram as shown in Figure 2.14. The hierarchical algorithms can be divisive or agglomerative [73]. Divisive approaches start with a single cluster that include all samples and perform division 
Table 2.2: Similarity metrics for hyperspectral data

\begin{tabular}{|c|c|}
\hline Metric & Equation \\
\hline Euclidean Distance (ED) & $d_{E D}=\sqrt{\left(s_{A}-s_{B}\right)^{T}\left(s_{A}-s_{B}\right)}$ \\
\hline Spectral Angle (SAM) & $d_{S A M}=1-\frac{s_{A}^{T} s_{B}}{\sqrt{\left(S_{A}^{T} S_{A}\right)\left(s_{B}^{T} s_{B}\right)}}$ \\
\hline Spectral Correlation (SCM) & $\begin{array}{c}d_{S C M}=1-\frac{\left(s_{A}-\bar{s}_{A}\right)^{T}\left(s_{B}-\bar{s}_{B}\right)}{\sqrt{\left(s_{A}-\bar{s}_{A}\right)^{T}\left(s_{A}-\bar{s}_{A}\right)} \sqrt{\left(s_{B}-\bar{s}_{B}\right)^{T}\left(s_{B}-\bar{s}_{B}\right)}} \\
\text { where } \bar{S}=\frac{\sum s_{i}}{n}\end{array}$ \\
\hline Spectral Information Divergence (SID) & $\begin{array}{l}d_{S I D}=\sum_{i=1}^{n} p_{i} \log \frac{p_{i}}{q_{i}}+\sum_{i=1}^{n} q_{i} \log \frac{q_{i}}{p_{i}} \\
\text { where } \mathrm{p}(\mathrm{i})=\frac{s_{A_{i}}}{\sum_{j=1}^{n} s_{A_{j}}}, \mathrm{q}(\mathrm{i})=\frac{s_{B_{i}}}{\sum_{j=1}^{n} s_{B_{j}}}\end{array}$ \\
\hline
\end{tabular}

operations. This is computationally expensive [73], and the agglomerative one starts with several clusters formed with one single sample, and then successive merge operations are performed using the similarity matrix until a single cluster is obtained with all samples.

To perform the merge operations, several criteria are used. The single linkage uses as the mini-

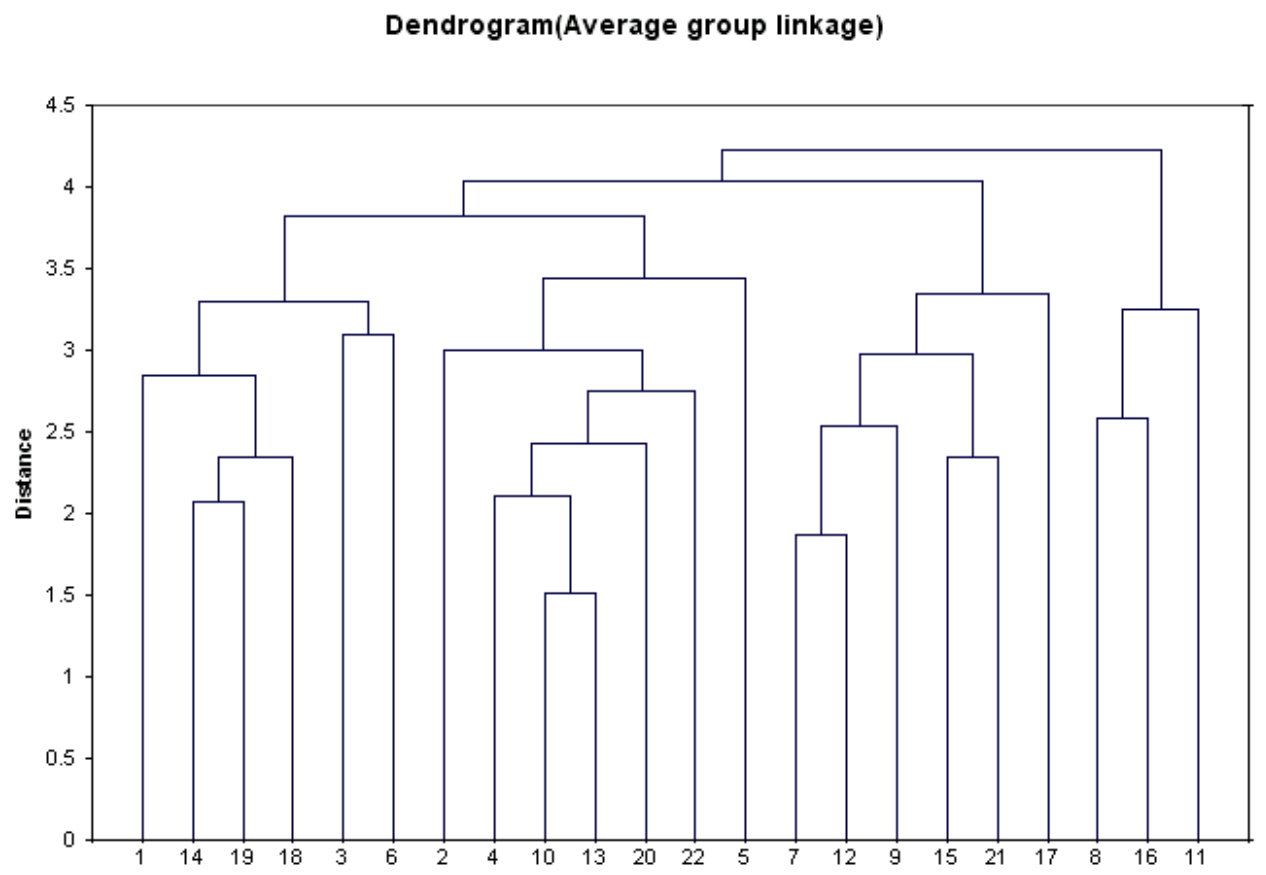

Figure 2.14: Example of Dendrogram for hierarchical clustering

mum distance between all pairs of samples from clusters. The complete linkage uses the maximum distance between all pairs of samples from the clusters. The average linkage uses the distance between centroids as the distance between clusters [72]. All the three methods are available in the 
statistics toolbox in MATLAB used in this work [77].

The Partitional approach, unlike hierarchical algorithms, obtains a single partition of the data which is of great advantage in large datasets [72]. Partitional clustering minimizes some criterion function to find the optimal partition of the data. The most common partitional algorithms are based on the squared error criterion [72] [73]. The most used partitional algorithm is k-means clustering that starts with a random partition and iteratively reassigns the samples to the clusters according to the similarity between the sample and the centroid of the clusters until convergence.

The Graph base approach uses a similarity graph $G=(\mathrm{V}, \mathrm{E})$, where each vertex represents a sample and the edges are built by the connection of very similar vertices. The problem of clustering can be viewed as the task of partitioning the graph $\mathrm{G}$ such that the connection between subgraphs are weak and the connection within subgraphs are strong [78]. In this approach, the edges are built using the $\varepsilon$-neighborhood graph that connects all points whose similarity are smaller than $\varepsilon$, or using the k-nearest neighbor graph that connects each vertex with its $\mathrm{k}$ closest vertices. The other type of graph is the fully connected graph that connects all points using a function (frequently used the Gaussian similarity function $e^{-\frac{\left\|x_{i}-x_{j}\right\|^{2}}{2 \sigma^{2}}}$ ) that hold the local neighborhood relations.

\subsection{Summary}

This chapter presents the state of the art of different methods for hyperspectral unmixing analysis that use the spatial-spectral information, it is evident that there is a need for more robust algorithms that use the spatial and spectral information contained in a hyperspectral image . Most of the spatial-spectral algorithms found in the literature use spatial kernels or windows into their procedures. Therefore, the obtained unmixing results depend on the size of these kernels. On the other hand, some algorithm employs unsupervised clustering techniques to determine spectrally uniform regions where endmember are extracted avoiding to use these kernels. However, similarly to most of existing spatial-spectral methods, algorithms do not use the spatial information for the estimation of the number of endmembers. In addition, does not perform the endmember extraction. This is a pre-processing step that determines a set of spectra that are passed to a spectral-only tech- 
nique for endmember extraction. Therefore, the development of new algorithms that use the spatial and spectral information of hyperspectral imagery for the combined estimation of the number of endmembers and their spectral signatures that do not use windows is essential. 


\section{Chapter 3}

\section{UNDERSTANDING SPATIAL-SPECTRAL DOMAIN INTERACTIONS IN HYPERSPECTRAL UNMIXING USING EXPLORATORY DATA ANALYSIS}

\subsection{Introduction}

Application of unmixing to a full HSI scene returns estimates of endmembers and their abundance that model the full spectral data cloud as the convex hull of the extracted endmembers. An important problem is that actual hyperspectral data clouds are not convex but better modelled as the union of convex distributions or approximated by piecewise-convex regions [20].

One cause for non-convexity of the data cloud in real hyperspectral imagery is that materials in a scene are not uniformly distributed so certain mixtures of materials are not possible due to spatial constraints. Here we use exploratory data analysis to analyze how spatial information can be used to extract the piecewise-convex regions in the image.

This chapter motivates an approach to perform unsupervised unmixing demonstrating how the spatial information helps to capture the relationship between the grade of uniformity of the clusters, and the convex regions in the image data set.

\subsection{Data Cloud Image Representation}

In this section, we illustrate, using both simulated and real hyperspectral data, how the spatial information helps to capture the relationship between the degree of uniformity and the convex regions in the image data cloud.

The simulated data consisted of a a scene with three different materials that are distributed in two scenarios; the first one is that they are randomly distributed in the scene where there is no spatial correlation between them, the other scenario shows that each material occupies a uniform 


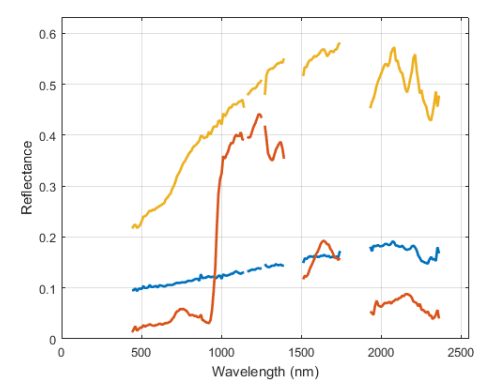

(a)

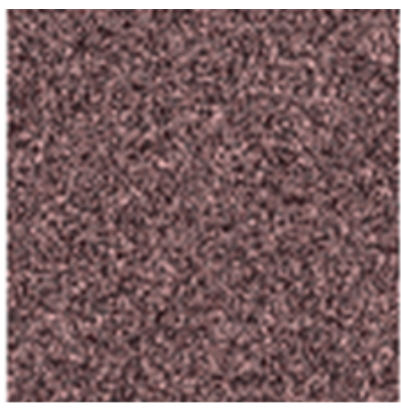

(b)

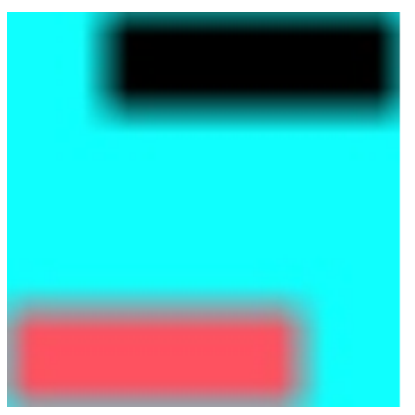

(c)

Figure 3.1: (a) Endmembers used in the simulated data; (b) RGB composite for spatially random distributed data; (c) RGB composite of image with spatial structure.

spatial region in the image where two materials are unmixed. Figure 3.1 shows the spectral signatures of the materials along with the two scenarios under consideration.

Figure 3.2 shows the first two principal components for each scenario. Figure 3.2(a) show the data cloud for the first scenario where there was no spatial correlation on the distribution of the materials in the scene. The data pixels here are all contained within the convex hull generated by the endmembers. Figure 3.2(b) shows the cloud for the structured scene. It is clear that the cloud pixels do not follow the convex structure like in 3.2(a). However, 3.2(b) can be considered as the union of two convex regions. The line between endmembers one and two and the line between endmembers two and three.

A $64 \times 64 \times 224$ image chip from the AVIRIS captured over Fort. A.P. Hill, Virginia USA in September of 2001 is used to illustrate what happens in a real data set. This image chip was selected because of the distribution to the information classes present and the spatial separation between the gravel field and the forest region which keeps these two classes from mixing. An RGB color composite is shown in Figure 3.3(a). The bands used for the color composite were 28 $(635.19 \mathrm{~nm})$ for Red, $19(548.09 \mathrm{~nm})$ for Green and $10(461.04 \mathrm{~nm})$ for Blue. The image has $64 x 64$ pixels with 224 spectral bands from 380 to $2500 \mathrm{~nm}$ with a spatial resolution of 3.5 meters. Only 197 bands are used here for the analysis as water absorption bands (bands 107 to 114, and 153 to 167) were removed.

The hypothesis formulated is that by decomposing the data cloud into convex regions we can 


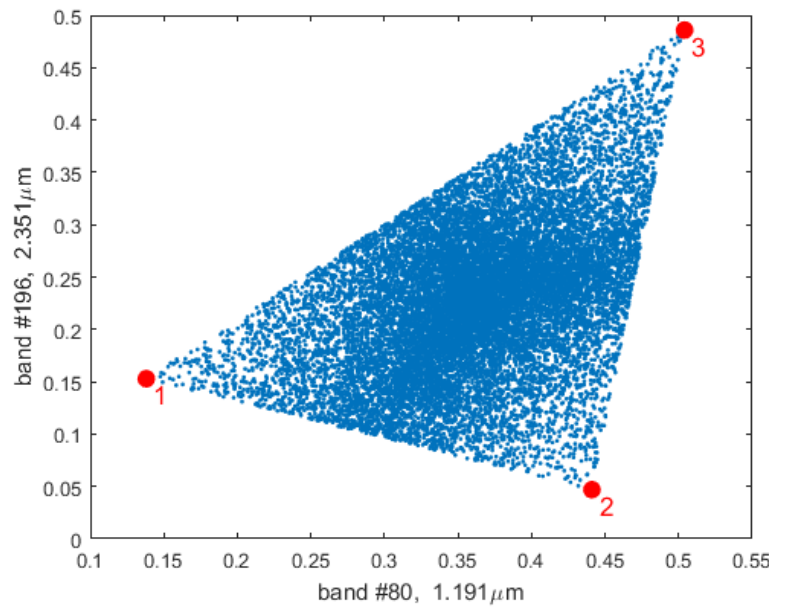

(a)

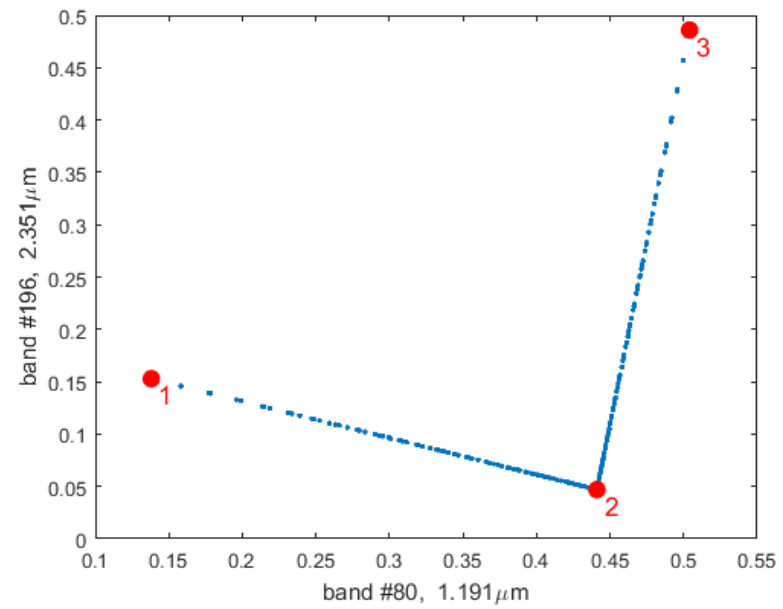

(b)

Figure 3.2: (a) Feature data cloud of the first scenario (materials are randomly distributed in the scene); (b) Feature data cloud of the second scenario (materials occupy some uniform spatial regions.

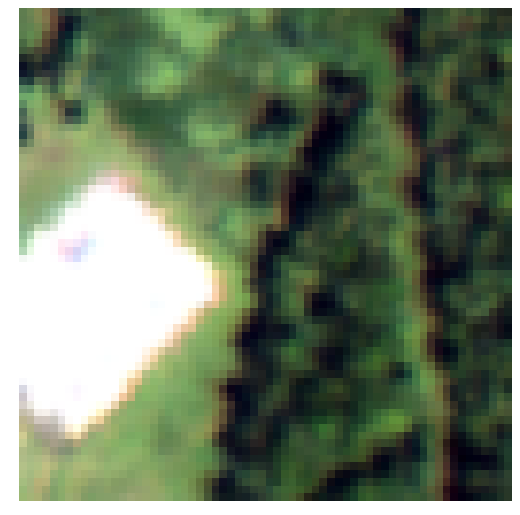

(a)

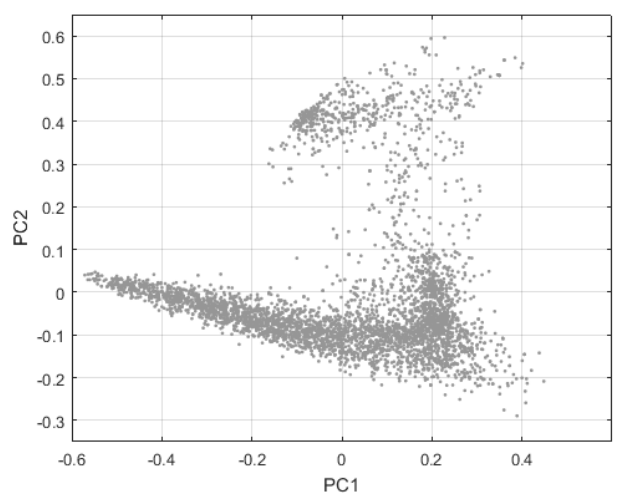

(b)

Figure 3.3: (a) True color RGB of a chip from AP Hill image; (b) Projection of the data points on the first 2 principal components.

obtain homogeneous regions in the image that may contain endmembers that can be easily captured when compared to global analysis.

\subsection{Partitioning the Data Cloud}

To visualize the data, similar to the simulated data, the hyperspectral image is projected into its first two principal components. Since they explain approximately $97.5 \%$ of the total variability. Scatter plots are generated using visualization capabilities of MATLAB. As shown in Figure 3.4(a), the 
2-D data cloud was manually segmented into three "convex" regions that correspond to the three main spatial segments in the image chip: parking lot (Figure 3.4(b)), grass field (Figure 3.4(c)), and forest (Figure 3.4(d)). The blue region represents the parking lot. It consists of two main endmembers, which are gravel and soil [8]. It will be easier to extract those two endmembers from this area than by looking at the whole image. Similar observation can be said regarding the third region that consists of summer deciduous forest and some shaded vegetation. However, there are clear limitations with this manual segmentation approach but for the purpose of the analysis presented here and to motivate our approach it suffices.

\subsection{Extracting Piecewise-Convex Structure Using Spatial Segmentation}

The previous section showed that by partitioning the data cloud into convex regions, we obtained spatial segments in the image. However, this approach is not practical since it's manual and highly dependent on user subjectivity. To illustrate the relation from spatial domain to extract the piecewise convext structure, we use coarse SLIC (Section 2.5.1) for image segmentation.

Figure 3.5 shows the result of segmenting the image chip using coarse SLIC with a region size of $25 \times 25$. The segmentation resulted in 9 segments. Figure 3.6 shows the location of each segment in the data cloud. An easy observation can be made in Figure 3.6(b), where the gravel field pixels are all located in the upper (convex) region in the cloud, similar conclusion can be made regarding the vegetation (forest) classes (Figures 3.6(d)-(i)) where most of these regions pixels are located at the lower side of the cloud. Clearly, spatial segmentation helps to capture elements of the piecewise convex structure of the data cloud. this was originally explored in [11], here we also use regional segmentation to improve endmember extraction for hyperspectral unmixing.

\subsection{Dimensionality Reduction Using Superpixel Segmentation}

Most of image processing techniques are pixel based, and do not take into account and information provided by the image, so if the image is large, the computation burden will be high. Also, the uniform pixel grid is not an efficient image representation as some information (spatial distribution 


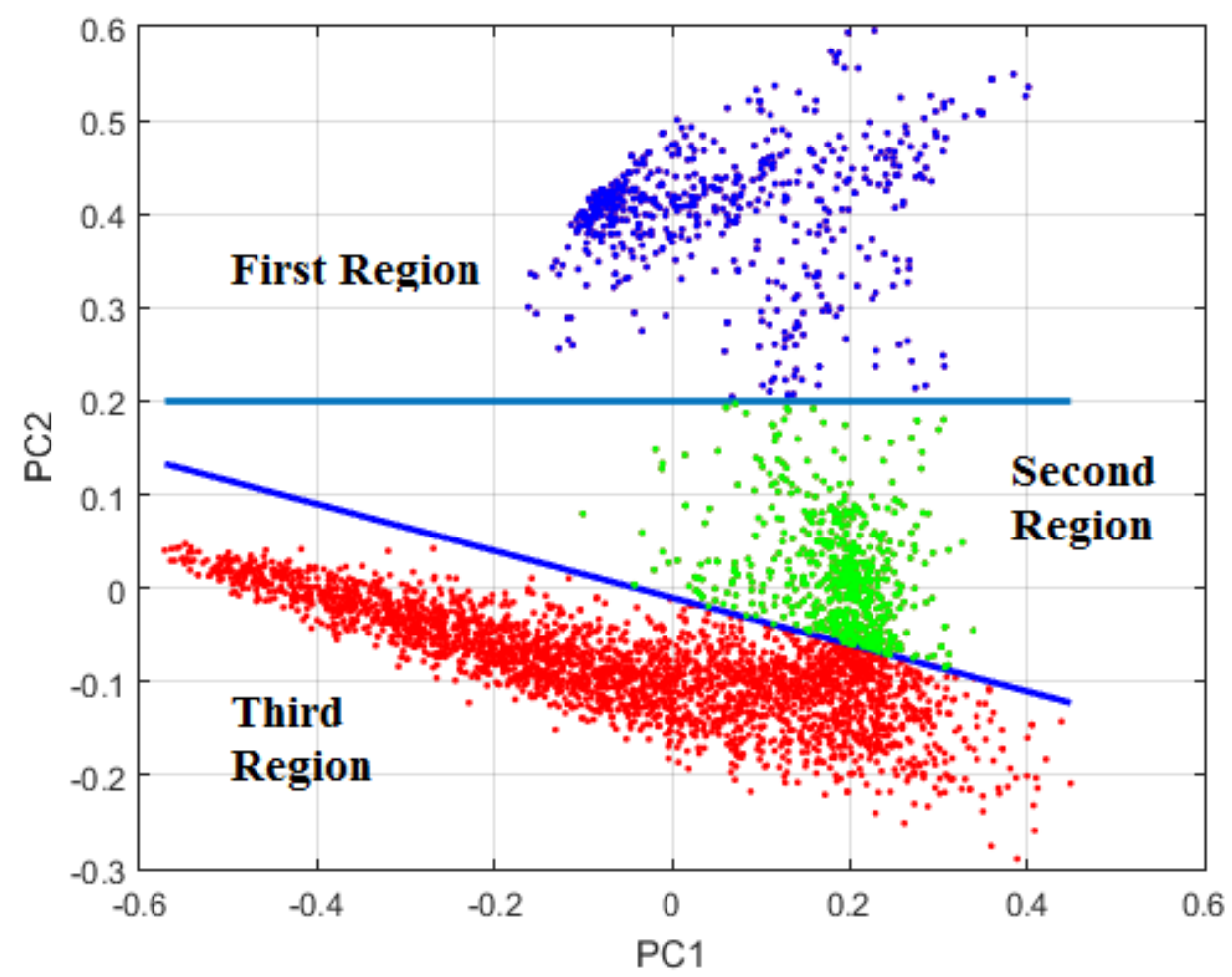

(a)

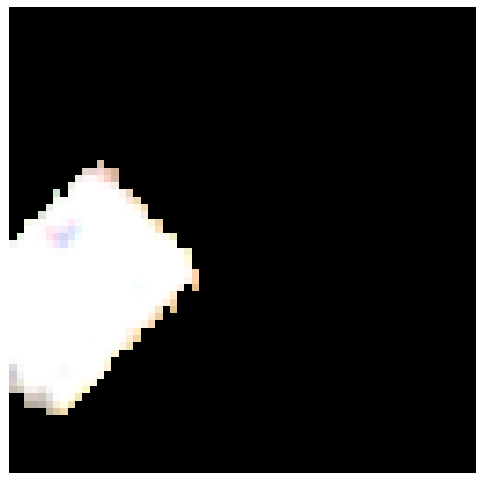

(b)

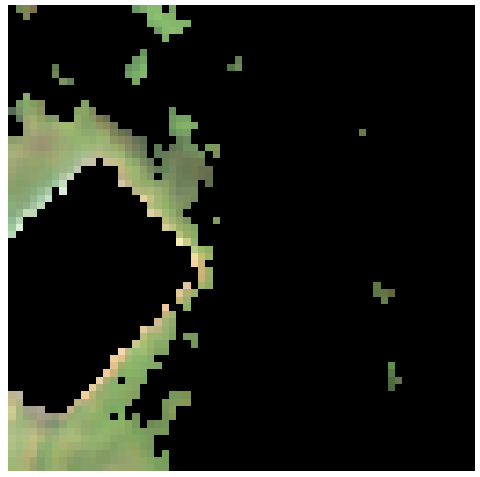

(c)

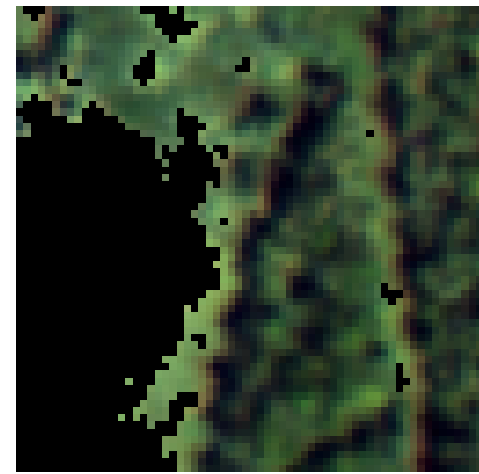

(d)

Figure 3.4: (a) Segmentation of the data cloud in feature space; (b) Region 1 in the spatial domain (Gravel field); (c) Region 2 in the spatial domain (Grass field); (d) Region 3 in the spatial domain (Summer deciduous forest).

of some materials) might not be uniformly spaced or distributed.

Superpixel representation breaks the image in locally homogeneous regions that are not necessarily distributed in a uniform grid. Each region could be treated as a single entity during processing. We propose the use of image spectral mean $\left(\mu=\frac{\sum_{i=1}^{n} x_{i}}{n}\right)$ of the spectral signatures to represent 


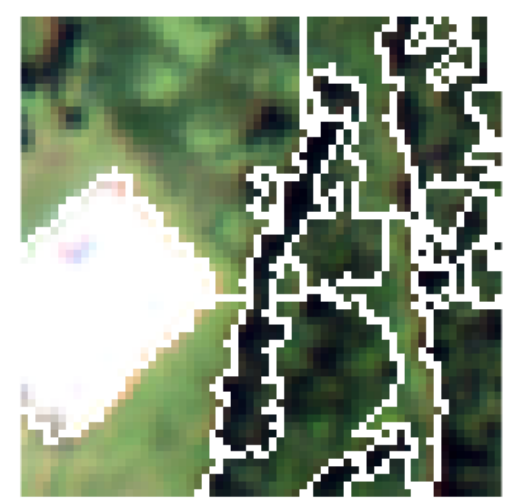

Figure 3.5: SLIC segmentation of AP Hill chip

each superpixel for the purpose of dimensionality reduction [79]. This will result in a lower dimensional (LD) representation that corresponds to a non-uniform sampling of the image.

Figure 3.7 shows where the SLIC centroids for each superpixel in Figure 3.6 are located on the data cloud. The centroids are numbered based on their appearance in Figure 3.6, for instance, centroid number 1 corresponds to 3.6(a), centroid 2 to $3.6(\mathrm{~b})$ and so on.

A better representation can be obtained by using a smaller region size. Figure 3.7(g) shows the centroids location on the cloud when the region size is 5. it is easy to see that the centroids data cloud keeps most of the features that are on the full data cloud.

Another illustration is displayed in Figure 3.7(e) and (h) where the RGB composition of the low dimensional images for both cases are presented. The SLIC segments form an $M \times N$ rectangular grid (See section 2.5.1 for details). So, by replacing each superpixel segment with the mean spectra of all pixels in that segment. A low dimensional hyperspectral cube is created. We then select the Red, Green and Blue bands to form an RGB composite of the image scene. Figure 3.7(e) corresponds to a LD image obtained using the $25 \times 25$ region size representation of the image. It clearly does not show rich spatial details as the original image (Figure 3.7(b)). While 3.7(h), capture many of the spatial features as in the original image. 

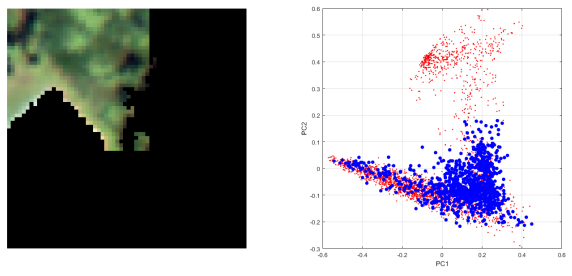

(a)
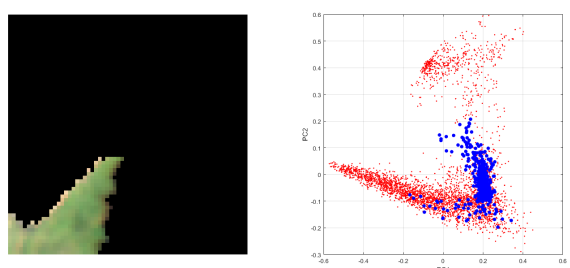

(c)
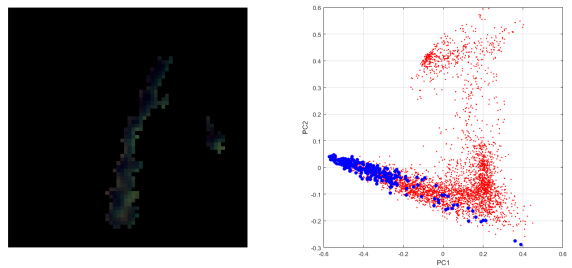

(e)
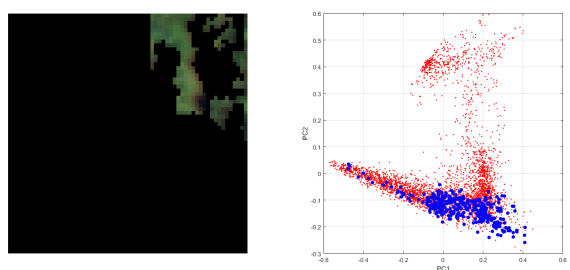

(g)
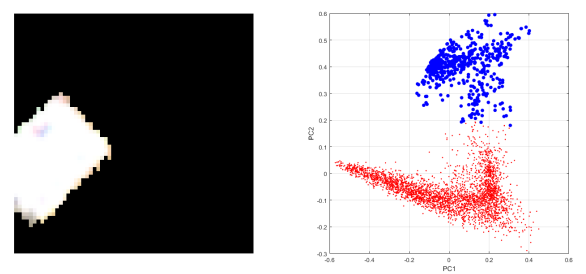

(b)
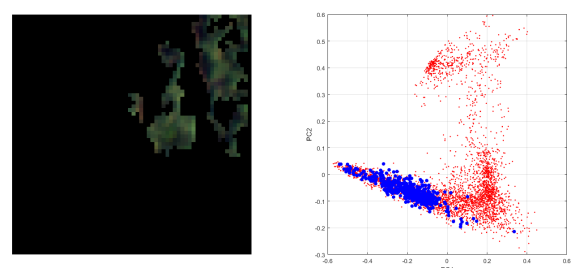

(d)
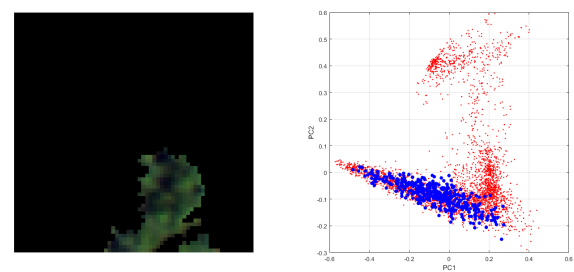

(f)
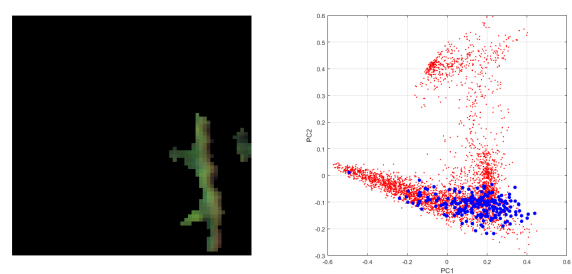

(h)
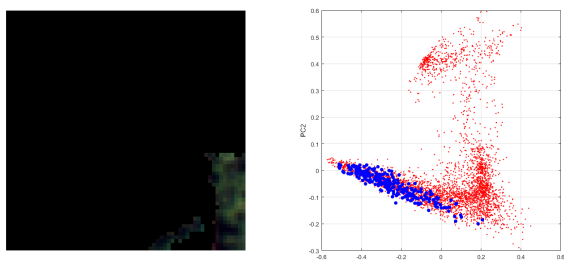

(i)

Figure 3.6: Result of SLIC Partitioning and their corresponding location in the cloud. 


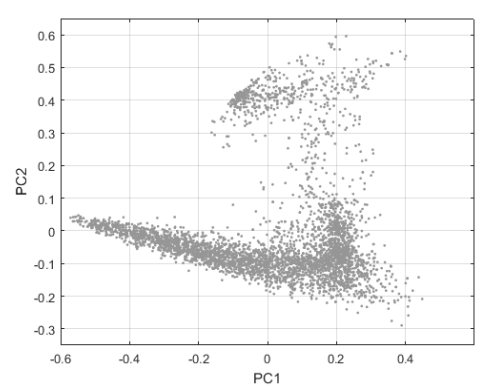

(a)

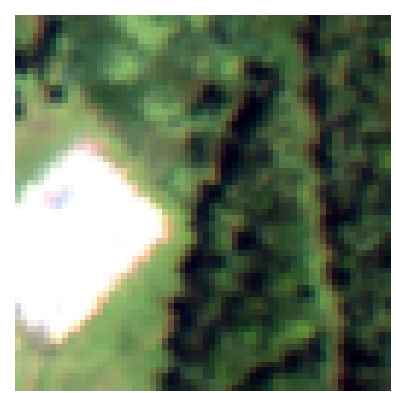

(b)

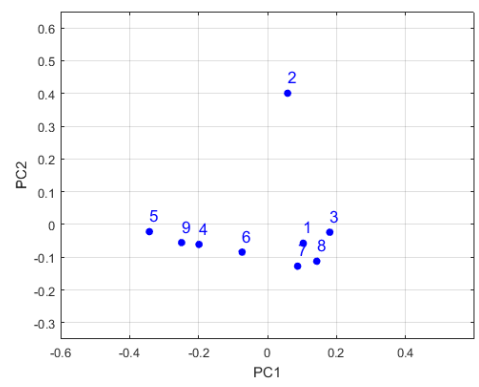

(c)

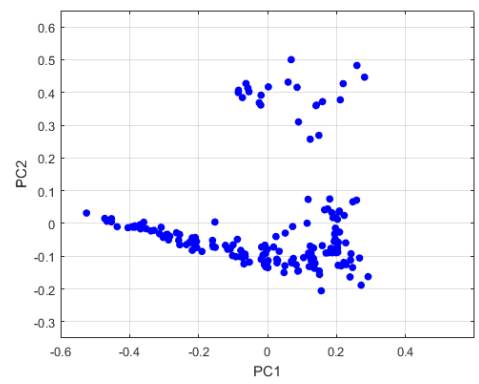

(f)

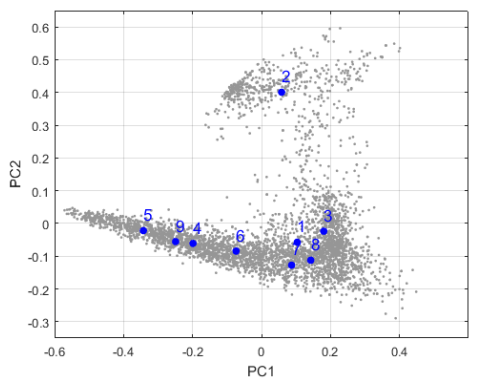

(d)

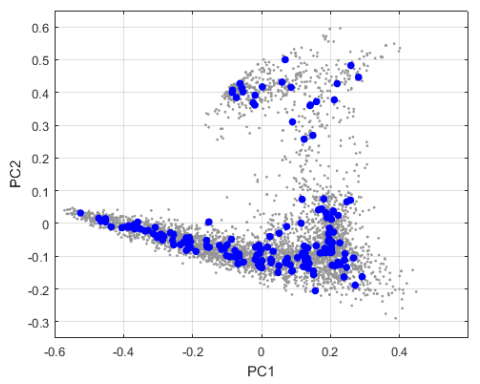

(g)

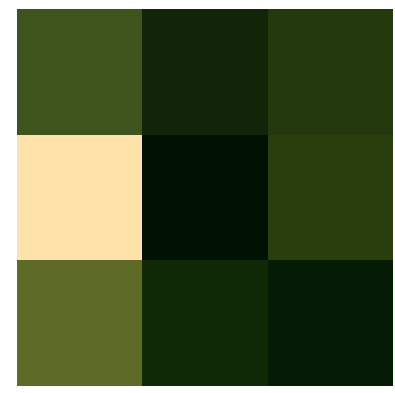

(e)

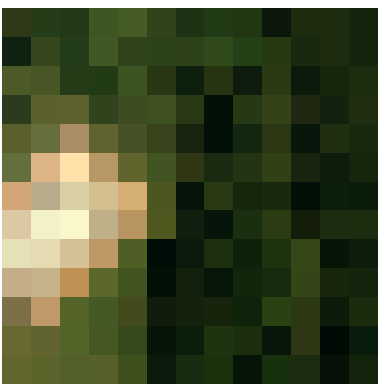

(h)

Figure 3.7: (a) Projection of the data points on the first 2 principal components; (b) RGB composite of the original image; (c) Superpixel centroids in feature space (region size $=25$ ); (d) Location of superpixel centroids on the data cloud; (e) RGB composite of the LD image; (f) Superpixel centroids in feature space (Region Size = 5); (g) Location of superpixel centroids on the data cloud; (h) RGB composite of the LD image.

\subsection{Summary}

In this chapter, we used visualization to understand the relation between the spatial domain information and data cloud structure. We saw experimentally that by partitioning the image into homogeneous regions we can capture some of the piecewise-convex structure of the cloud.

Dimentionality reduction is the main idea behind the superpixel algorithm. By decomposing 
the image into superpixels, we can reduce the dimensionality of the image, while maintaining the convex structure of the cloud and representing each superpixel with its spectral mean.

The proposed approach will combine segmentation and superpixel-based dimensionality reduction in unmixing. 


\section{Chapter 4}

\section{PROPOSED ALGORITHM}

\subsection{Introduction}

Figure 4.1 shows the different components of the proposed Superpixel-Based Hyperspectral Unmixing with regional segmentation. The algorithm is an unsupervised hyperspectral unmixing process that takes advantage of the spatial information of the image.

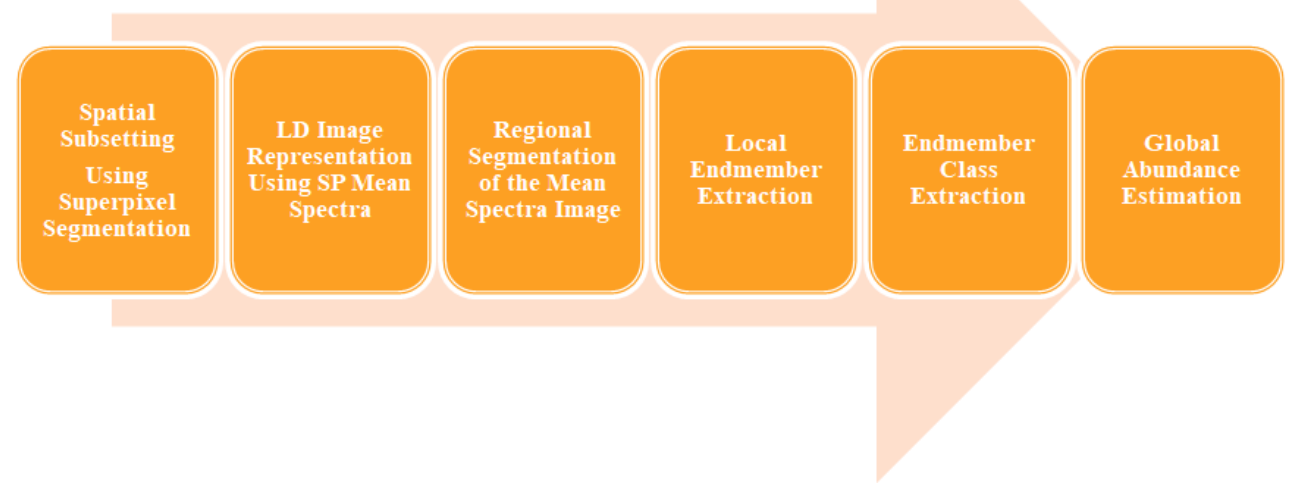

Figure 4.1: Proposed superpixel-based unmixing

First, superpixel segmentation is applied to the image. A low dimensional representation of the image is created by representing each superpixel by its mean spectra. Second, the LD superpixel image is segmented into regions using quad-tree segmentation. Endmember extraction is applied to each region to extract regional endmembers. Extracted endmembers are clustered into endmembers classes. Abundances are computed over the full image using the extracted spectral endmembers.

\subsection{Dimensionality Reduction Using SLIC}

The value of the superpixel representation in image processing and computer vision has been recognized in the literature. Simple linear iterative clustering (SLIC) [58] algorithm showed better 
performance in superpixel segmentation than other methods in terms of computation speed and memory efficiency [58]. Here SLIC is used for superpixel segmentation in the proposed unmixing method.

The Simple Linear Iterative Clustering (SLIC) is a simple method to decompose an image into homogenous regions. It is based on a spatially localized version of the k-means clustering.

SLIC starts by dividing the image into an equally spaced grid as shown in Figure 4.2(a). The center of each grid tile is then used to initialize a corresponding k-means clustering. After kmeans, SLIC optionally removes any segment whose area is smaller than a predefined threshold by merging them into larger superpixels. The details of the algorithm were described in Section 2.5.1. Figure 4.2(b) shows the final result of the segmentation.

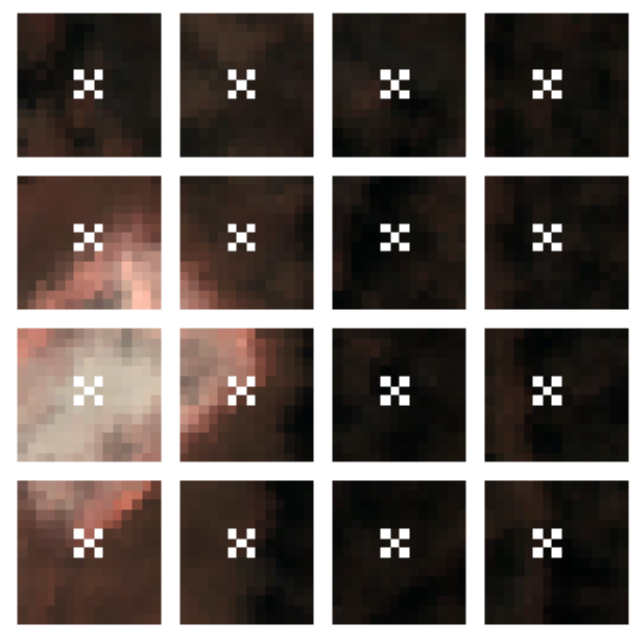

(a) Dividing the Image into Tiles.

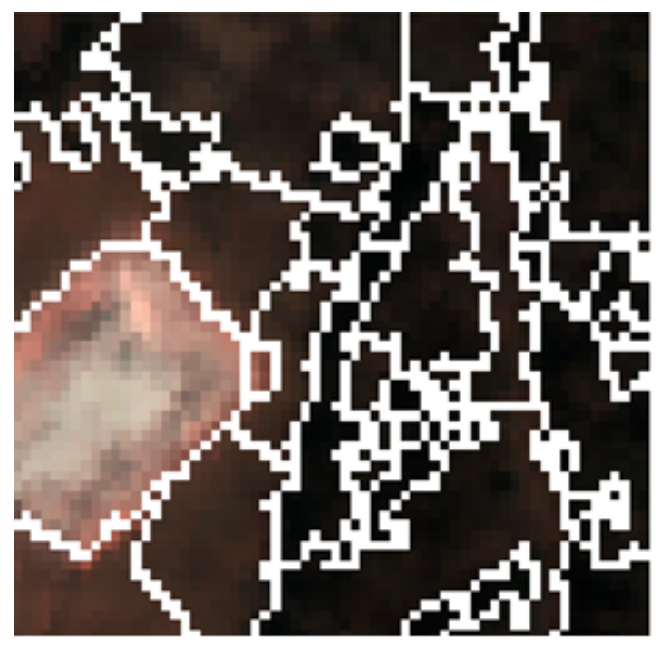

(b) Final Segmentation.

Figure 4.2: SLIC Segmentation.

Figure 2.10 shows SLIC segmentation for a chip from AVIRIS Fort A.P. Hill scene (see Section 2.6), for different sizes. Notice that the number of superpixels decreases as the region size is increases. A point to study is to find the optimal value of the region size parameters that will give the best representation of the image. As the region size becomes smaller, the image will become highly over-segmented and we may lose the dimensionality reduction advantage. On the other hand, as the region size becomes larger, the superpixel representation won't accurately describe the image as non-homogeneous superpixels might occur. In this research, we consider a region 
value of $16 \times 16$ as it was used by [62].

To obtain a lower dimensional (LD) representation of the image. Each SLIC superpixel is represented by the mean spectra of the spectral signatures in the superpixel [79]. The result is a low dimensional (LD) representation that corresponds to a non-uniform spatial sampling of the image.

\subsection{Quadtree Segmentation}

The LD-SLIC image is divided into spectrally uniform regions using quadtree partitioning (see Section 2.6.3). The spatial subsetting makes the approach more sensitive to endmembers present in small regions when compared to a global approach that assesses the full image at once. To determine the appropriate number and size of the spatial subsets for in the quadtree a given image, we use two splitting criteria. First, the maximum level of the quadtree partition (16 parts). Second, reduction below $90 \%$ of the Shannon Entropy (2.16) for the full image as explained in [63].

\subsection{Endmember Extraction}

After quadtree partitioning, the next step is to extract the endmembers from each leaf tile. Most endmember extraction methods assume the number of endmembers is known. For this reason, the estimation of the number of endmembers also has to be considered as part of the unmixing problem. Approaches commonly followed in hyperspectral unmixing to determine the number of endmembers were described in Section 2.4.2.

\subsubsection{Estimation of the Number of Endmembers Using the Gram Method}

The Gram Matrix [50] [49], $G$, is an $m \times m$ matrix where $m$ is the number of vectors in the test set, $\left.\mathbf{Y}_{i}\right|_{i=1} ^{m}$. First, the column of $\mathbf{Y}$ are sorted in a descending order based on their magnitude. The Gram matrix is given by:

$$
G=\mathbf{Y}^{T} \mathbf{Y}
$$


The $\{i, j\}_{t h}$ element of the Gram Matrix is given by the inner product between the $i_{t h}$ and $j_{t h}$ vectors $y_{i}, y_{j}$. One unique property of the Gram Matrix is that the determinant, the Gramian, is the square of the volume of the parallelepiped formed by the vectors of $\mathbf{Y}$ [51]. Also, the vectors are linearly independent if and only if the Gramian is nonzero. Here, we compute the principal minors of the Gram Matrix starting with the $3 \times 3$ value and keep going until the determinant is zero. The vectors in $\mathbf{Y}$ are ordered from highest to lowest magnitude, the magnitude of the principal minors peaks at a small number of vectors and then monotonically decreases.

In hyperspectral images, the matrix $\mathbf{Y}$ is a subset of m-pixels from the original image $\mathbf{X}$. The value of $\mathrm{m}$ is an overestimate of the expected number of endmembers in the scene. The set of pixels in $\mathbf{Y}$ can selected using endmember extraction techniques (SVDSS [80], VCA [27], PMF [30]...etc.).

\subsubsection{Endmember Extraction Using SVDSS}

Endmember extraction will be performed on each quadrant using the Singular Value Decomposition Subset Select (SVDSS). SVDSS is an algorithm used to select the most independent subset of columns from a matrix. It has been applied to band subset selection in the past [81].The SVDSS selection algorithm for endmember extraction is summarized as follows [80]:

1. Construct a matrix representation $\mathbf{X}$ of the hyperspectral image. Each column of $\mathbf{X}$ is the spectral signature of each pixel in the hyperspectral image.

2. Compute the Singular Value Decomposition (SVD) [82] of $\mathbf{X}=\mathbf{U} \Sigma \mathbf{V}^{T}$.

3. Compute the $\mathrm{QR}$ factorization with pivoting of the matrix $\mathbf{V}_{p}^{T} \mathbf{P}=\mathbf{Q R}$ where $\mathbf{V}_{p}$ is formed by the first $\mathrm{p}$ right singular vectors of $\mathbf{X}$ and $\mathrm{p}$ is the number of endmembers.

4. Let $\mathbf{X P}=\left[\mathbf{X}_{1} \mid \mathbf{X}_{2}\right]$ where $\mathbf{X}_{1} \in \mathfrak{R}^{m \times p}$ and $\mathbf{X}_{2} \in \mathfrak{R}^{m \times(N-p)} . \mathbf{X}_{1}$ will be the matrix of endmembers

When comparing the SVDSS algorithm to the VCA algorithm, SVDSS always give the same set of endmembers at each run, while the VCA gives a different set of endmembers due to its random 
nature [27]. In order to reduce the randomness of VCA, authors in [83] run the VCA 10 times the take the solution that forms a simplex with the largest volume (Eq. 2.3). Also, it is shown in [80] that SVDSS results in the vectors with a large simplex volume.

\subsection{Endmember Class Extraction}

A large number of spectral endmembers can be extracted from all the tiles in the image. These spectral endmembers represent the distinct components in a scene as well as their spectral variability. Thus, it is necessary to group spectral endmembers into sets with similar spectral features, i.e. meaningful clusters that represent the distinct materials and their variability in the image. These meaningful sets of spectral endmembers are the spectral endmember classes.

To build the spectral endmember classes, clustering techniques (Section 2.7) are used to group spectral endmembers signatures into sets with similar spectral features to represent the same material. In our research, we used the spectral angle measure (SAM) (see Table 2.2) as our similarity measure. If the distance between two endmembers is less than 0.005 the endmembers will belong to the same spectral endmember class. The principal advantage of SAM clustering is that it does not need a specific number of clusters. We used the statistics toolbox [77] available in MATLAB to perform the clustering analysis.

\subsection{Abundance Estimation}

Once the endmember classes are determined, the next step is to estimate their abundances. The abundances are computed by solving the abundance estimation problem (see Section 2.4.3) using all spectral endmember signatures.

The abundances satisfy non-negativity and sum less than or equal to one (NNSLO) constraint. The reason for using NNSLO constraint instead of the sum-to-one (NNSTO) constraint is to account for topography effects as described in [52].

The abundance of an endmember class is the sum of the abundances of the spectral endmember 
signatures belonging to that class. Constrained least squares are used to compute the abundance as described in Section 2.4.3.

\subsection{Summary}

This chapter described the proposed approach. Our method starts by segmenting the image into homogeneous regions using the SLIC algorithm, we took advantage of the available MATLAB code in [84] to perform the task. Once the SLIC segments are obtained, dimensionality reduction is achieved by representing each superpixel with its mean spectra as suggested in [79]. Regional segmentation over the LD image is performed using the quadtree algorithm. Endmember extraction using SVDSS [80] is performed over each tile of the quadtree. The extracted spectral endmembers were clustered using the cosine distance to form information classes, we used the available statistics toolbox from MATLAB to perform the task. Abundance estimation using the NNSLO [52] is performed over the scene to obtain the abundance maps.

The next chapter shows experimental results on different real data sets. 


\section{Chapter 5}

\section{EXPERIMENTS WITH REAL HSI DATA SETS}

\subsection{Introduction}

This chapter presents experiments to evaluate the performance of the super-pixel based unmixing with regional segmentation approach using real hyperspectral image data sets. Two images are used in this chapter: HYDICE Urban and ROSIS Pavia University. A detailed description of these images is presented in Section 5.2. In Section 5.3, we compare our approach using quantitative and qualitative analysis with other approaches used in the literature. Section 5.4 shows a summary of the results.

\subsection{Data Sets}

Two hyperspectral images were selected for the experiments. HYDICE Urban and ROSIS Pavia University. The reason of the data selection is because of the availability of reference data in the format of published spectral libraries and classification maps that allow assessment of unmixing results.

\subsubsection{Urban Data Set}

Urban is one of the most widely used hyperspectral data used in the hyperspectral unmixing studies. It was recorded by the HYDICE (Hyperspectral Digital Image Collection Experiment) sensor in October 1995. The scene is an urban area at Copperas Cove, TX, U.S [85]. There are $307 \times 307$ pixels at $2 \mathrm{~m}$ resolution. In this image, there are 210 bands between $400 \mathrm{~nm}$ to $2500 \mathrm{~nm}$, at a spectral resolution of $10 \mathrm{~nm}$. After the channels 1-4, 76, 87, 101-111, 136-153 and 198-210 are

removed (due to dense water vapor and atmospheric effects), we end with 162 channels. Figure 5.1(a) presents the RGB composition using bands $28(680 \mathrm{~nm})$ for red, band $14(540 \mathrm{~nm})$ for green and band $7(470 \mathrm{~nm})$ for blue. A classification map from [7] is used here as a reference and shown 


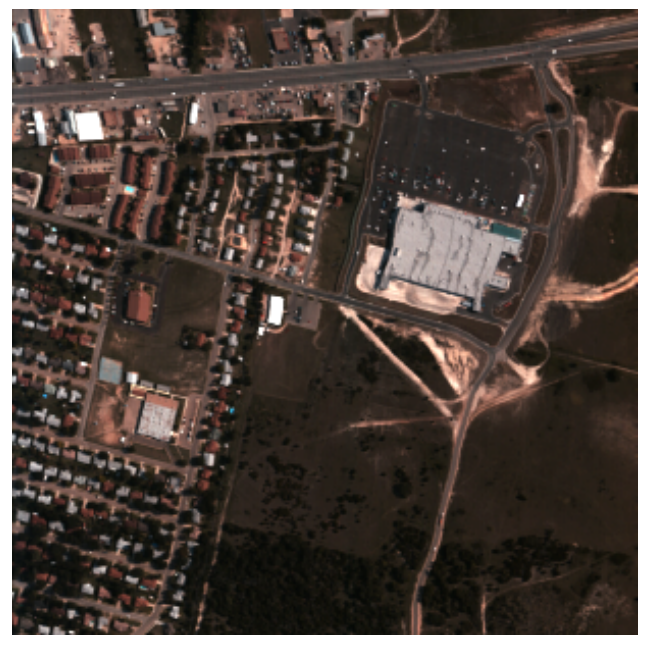

(a)

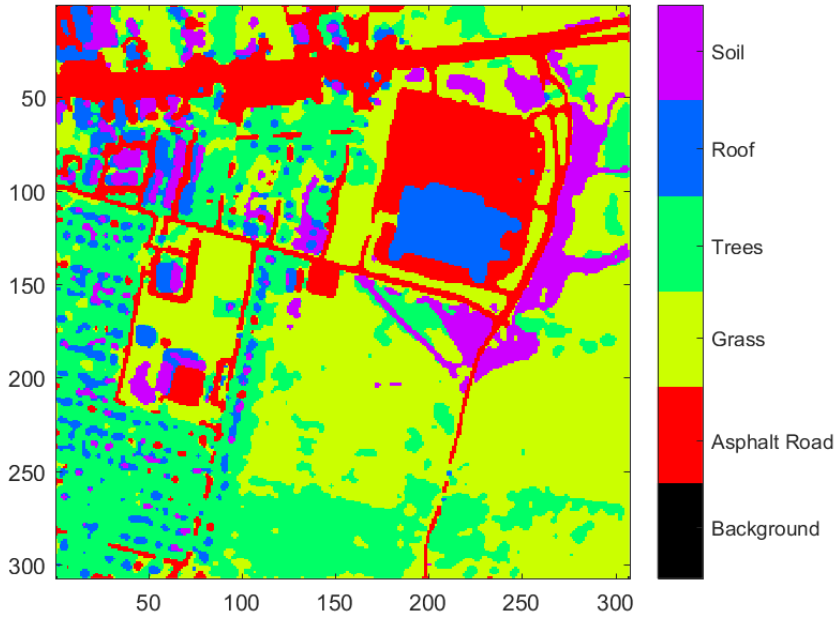

(b)

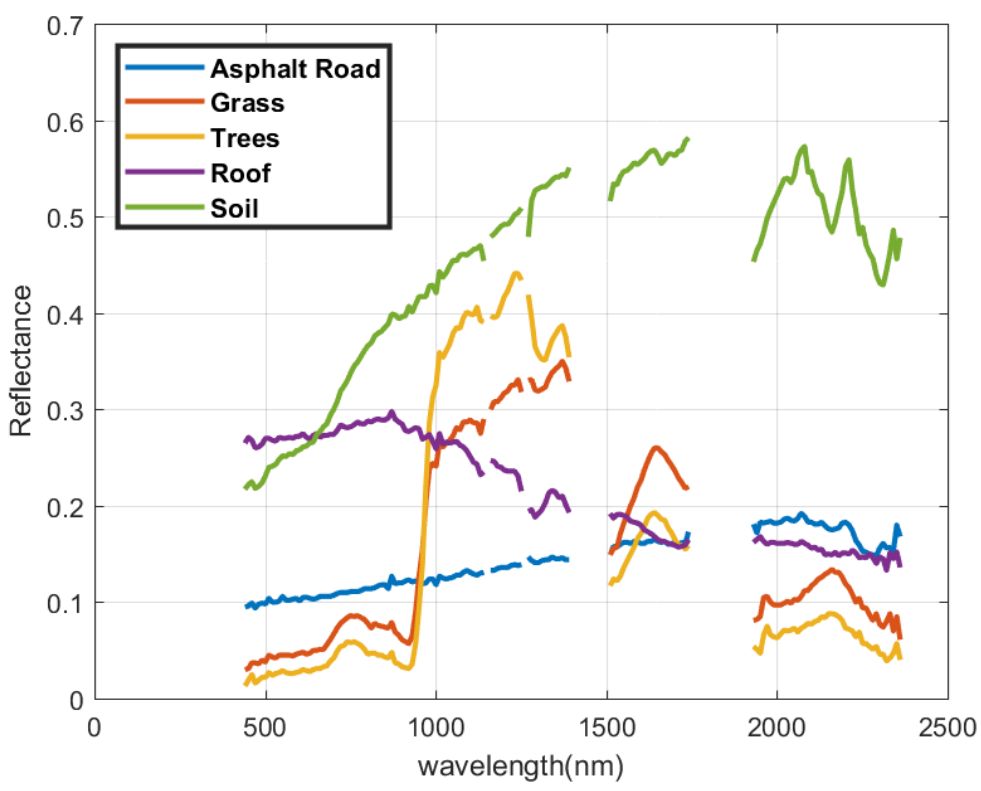

(c)

Figure 5.1: Urban Image: (a) true color RGB composite; (b) Reference data classification map [7]; (c) Urban Endmembers.

in Figure 5.1(b). The classification map shows 5 different classes: Asphalt, Grass, Tree, Roof and Soil (Dirt). The endmember spectral library is also available from [7] and it's shown in Figure 5.1(c). 


\subsubsection{Pavia University}

This is a scene acquired with the ROSIS sensor during a flight campaign over Pavia, northern Italy. The image consists of 103 spectral bands from 430 to $860 \mathrm{~nm}$ with a spectral resolution of $4 \mathrm{~nm}$ and a spatial resolution of 1.3 meters. Only 103 bands are used for the unmixing analysis. Pavia University is $610 \times 340$ pixels. Figure. 5.2(a) shows an RGB composite of the scene. The reference classification map for the Pavia University scene is shown in Figure 5.2(b). The classification map shows 9 classes: Asphalt, Meadows, Gravel, Trees, Metal Sheet, Bare Soil, Bitumen, Brick and Shadow. The unlabeled samples in the image are colored in black and labeled as "background". Figure 5.2(c) shows the endmembers for the labeled samples.

\subsection{Assessment Methods}

Most of the time, hyperspectral datasets have documented classification maps and spectral libraries such as Fort A.P. Hill, HYDICE Urban, and ROSIS Pavia images. But, there is no ground truth about abundance maps. The lack of this information becomes a great limitation for any effort to determine the reliability and accuracy of unmixing results. Commonly, unmixing results are evaluated by computing the spectral angle between the extracted endmembers and spectral references, and using the reconstruction error that compares the image reconstructed from the estimated abundances and endmembers and the original image [86]. But, the comparison between extracted endmembers and spectral references is limited to the fact that the extracted endmembers are imagederived signatures and the spectral references correspond usually to laboratory spectra. In addition, the reconstruction error only assess the fitting error but not the accuracy of the estimated abundance maps or endmembers.

In this work, we will follow the assessment methodology proposed by [38] that use the information from a classification map. It is expected that a single or a combination of spectral endmember classes can be related to information classes within a classification map. Using these relations, a quantitative assessment methodology can be performed to determine how well the unmixing algo- 


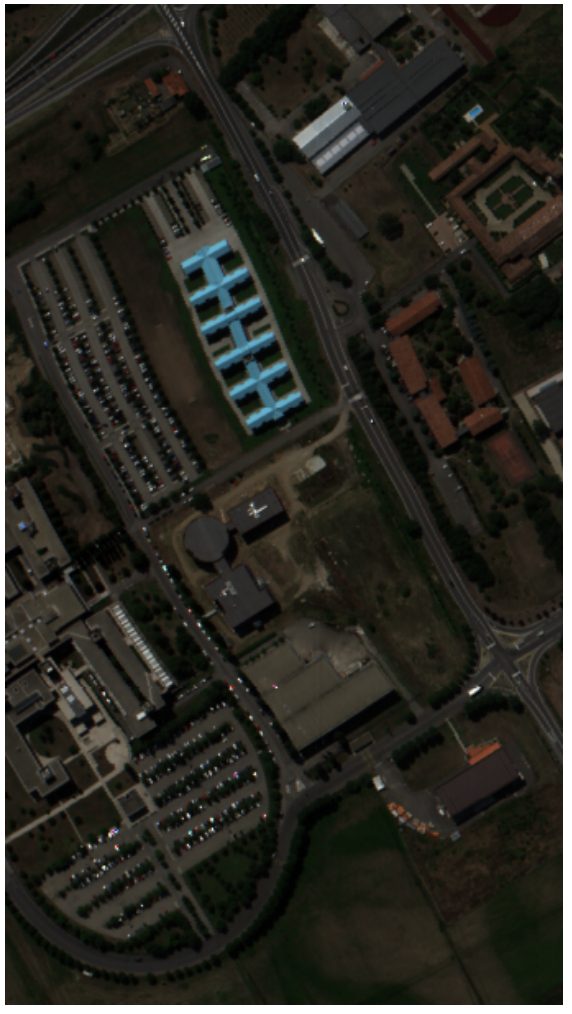

(a)

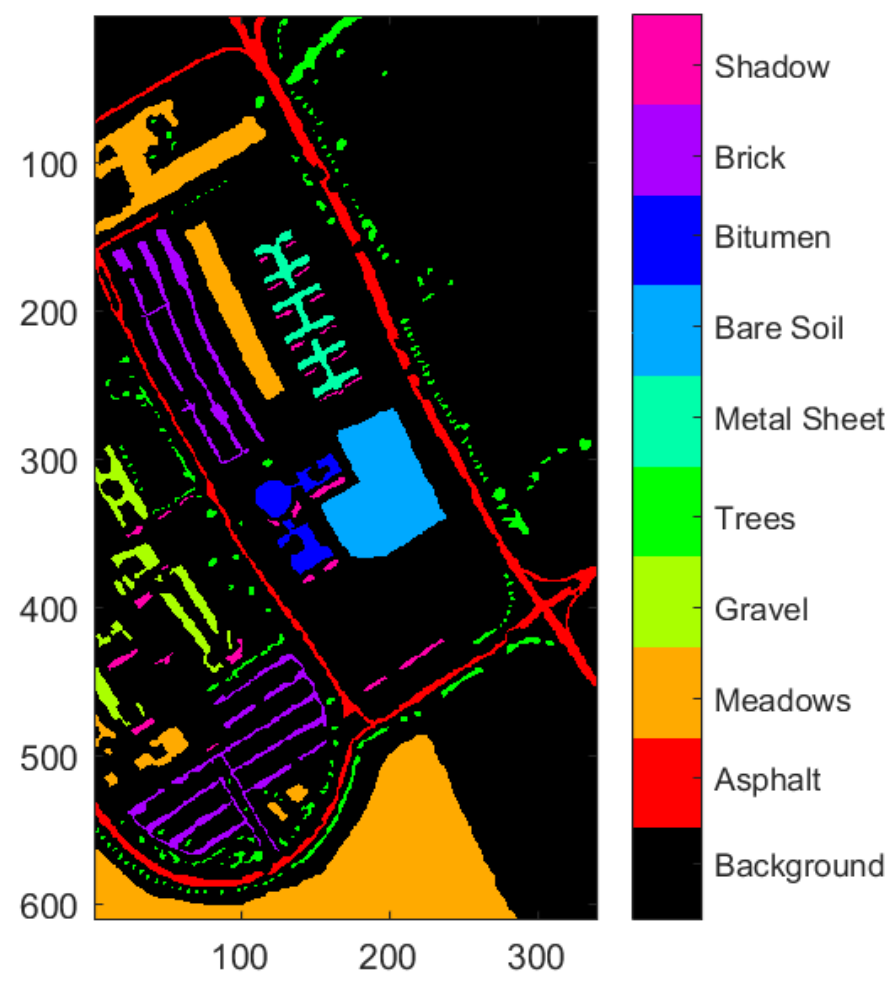

(b)

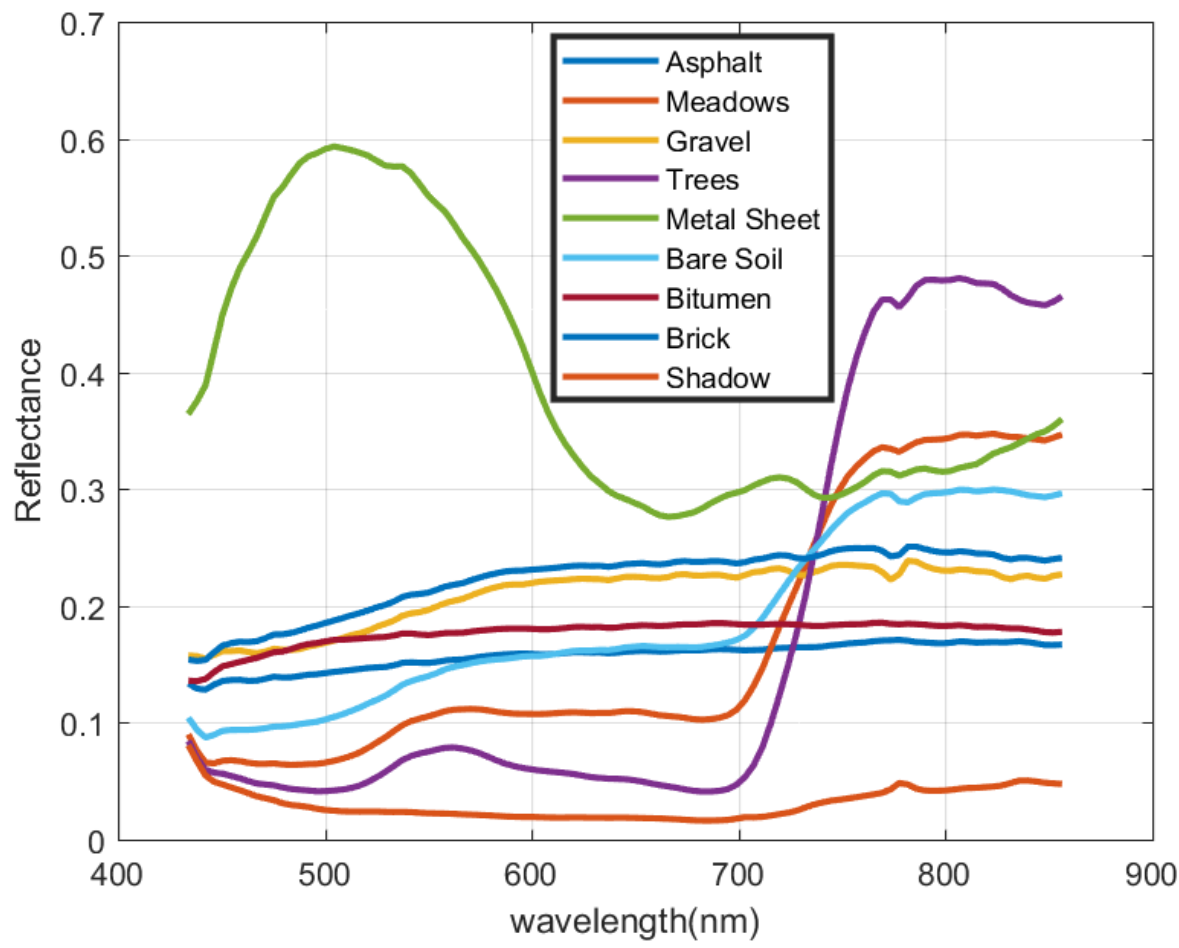

(c)

Figure 5.2: ROSIS Pavia University (a) true color composite; (b) Reference calss map; (c) Endmembers. 
rithm detects the distinct components of an image. The methodology follows two ways to assess the unmixing results. The first one is inspired in detection theory and seeks to establish if the materials that are part of an information class are detected or not. The second is based on classification accuracy analysis. A classification map is obtained using the estimated abundances and majority vote criterion to assign a label. An agreement analysis between the generated classification map and the reference map is performed using the confusion matrix. Before performing the quantitative assessment, it is necessary to identify the relation between spectral endmember classes and information classes. For that, a qualitative comparison between abundances and reference classification maps is performed. The next sections describe each step of the assessment methodology.

\subsubsection{Qualitative Evaluation}

The qualitative evaluation relates the spectral endmember classes with the information classes. For that, two analyses are conducted. First, a comparison of the spectral endmember classes with available spectral libraries from the distinct images is performed. The comparison is done using the shape of the spectral signatures, and validated using the spectral angle (2.2) when the reference spectra is available. Second, abundance maps are compared to classification maps. The classification maps allow to know the different components of an image, and these provide some information about the spatial distribution of the materials. Then, it can be expected that similar distribution are obtained in the estimated abundance maps. This step of the assessment methodology requires the intervention of an image analyst.

Note that one or more spectral endmember classes can be related to one or more information classes. For the next step in the assessment methodology, spectral endmember classes related to the same information classes are combined, as well as, if an extracted spectral endmember class is related to several information classes, then the information classes are combined into a single class. Recall that the proposed approach is a machine-based approach so it may confront difficulties in separating spectrally similar classes. 


\subsubsection{Quantitative Assessment of Detected Classes}

A majority vote criterion is used to generate a classification map from the estimated abundance maps. This map is compared with the reference map using an agreement matrix (Confusion Matrix). Four statistics are computed from the agreement matrix: (1) The Producer's Accuracy refers to the probability that a certain land-cover of an area on the ground is classified correctly, (2) the User's Accuracy which is the probability that a pixel labeled as a certain land-cover class in the map is really in that class, (3) the Overall Accuracy that is indicated the percentage of pixels with the same label in both the reference map and the new classification map, and (4) Cohen's kappa coefficient [87].The Kappa statistic is a more robust measure than simple percentage agreement calculation, as it takes into account the possibility of the agreement occurring by chance.

\subsubsection{The Confusion Matrix}

In the field of machine learning, a confusion matrix, also known as an error matrix [88] is a specific table layout that allows visualization of the performance of classification algorithm. Each row represents the results of the classification while each column represents the true class for the labeled samples. The name comes from the fact that it makes it easy to see if the classifier is confusing two classes (or mislabeling one as another).

Table 5.1 shows an example of a confusion matrix of 3 classes (Water, Soil and Trees). Assuming a sample of 67 pixels of water, 23 pixels of Soil and 10 pixels as Trees.

In the confusion matrix, of the 67 actual water pixels, the classifier predicted 50 would be Water, 14 would be Soil, and 3 as Trees. That means 50 out of the 67 Water pixels were correctly classified, to calculate the Producer's Accuracy for this class, the result will be 50/67 $=0.75$ or 75\%. Similar analysis could be done to calculate the Producer's Accuracy for the other 2 classes and the results shown in Table 5.1. The confusion matrix indicates that the resulting class map has 57 pixels labeled as Water, 27 as Soil and 16 as Trees, the User's Accuracy for Water would be $50 / 57=0.88$, or simply $88 \%$. The Overall Accuracy is the ratio of correctly classified pixels over the total number of pixels, in this case $71 / 100=0.71$ or $71 \%$. 
Table 5.1: Example of a Confusion Matrix

\begin{tabular}{|c|c|c|c|c|c|c|}
\cline { 3 - 7 } \multicolumn{2}{c|}{} & \multicolumn{3}{c|}{ Reference Data } & \multicolumn{2}{c|}{} \\
\cline { 3 - 7 } \multicolumn{2}{c|}{} & Water & Soil & Trees & Totals & $\begin{array}{c}\text { User's } \\
\text { Accuracy (\%) }\end{array}$ \\
\hline \multirow{3}{*}{ Class Map } & Water & 50 & 5 & 2 & 57 & 88 \\
\cline { 2 - 7 } & Soil & 14 & 13 & 0 & 27 & 48 \\
\cline { 2 - 7 } & Trees & 3 & 5 & 8 & 16 & 50 \\
\hline \multirow{2}{*}{ Totals } & 67 & 23 & 10 & 100 & Overall \\
\cline { 2 - 7 } & $\begin{array}{c}\text { Producer's } \\
\text { Accuracy (\%) }\end{array}$ & 75 & 57 & 80 & & Accuracy $=\mathbf{7 1 \%}$ \\
\hline
\end{tabular}

Table 5.2: Meaning of the Value of Kappa [10]

\begin{tabular}{|c|c|}
\hline Value of Kappa & Agreement \\
\hline Negative & Poor \\
\hline $0 \leq \kappa \leq 0.2$ & Slight \\
\hline $0.21 \leq \kappa \leq 0.4$ & Fair \\
\hline $0.41 \leq \kappa \leq 0.6$ & Moderate \\
\hline $0.61 \leq \kappa \leq 0.8$ & Substantial \\
\hline $0.81 \leq \kappa \leq 1.00$ & Almost Perfect \\
\hline
\end{tabular}

The Producer's Accuracy and the User's Accuracy show how well the classifier is working in a per-class manner and do not show the overall performance of that classifier, while the Overall Accuracy is just a summary (or an average) that does not reveal if error was evenly distributed between classes or if some classes were really bad and some really good. This is more easy to see in Producer's and User's accuracies.

The fourth statistic that will be used to evaluate the quality of the classifier is the Kappa Coefficient $(\kappa)$ which is a statistical measure of the agreement, beyond chance, between two decisions (e.g. classifier and reference data). It reflects the difference between actual agreement and the agreement expected by chance. Mathematically, $\kappa$ can be calculated by [10]:

$$
\kappa=\frac{\operatorname{Pr}(\text { correct_classification })-\operatorname{Pr}(\text { chance_classification })}{1-\operatorname{Pr}(\text { chance_classification })}
$$

For the above example, $\kappa$ equals 0.4630 , this means that the classifier has $46.3 \%$ better agreement than by chance alone. Table 5.2 shows the meaning of different values of kappa [10]. 


\subsection{Results}

Urban and Pavia are analyzed using the approach in Figure 4.1. First, the SLIC method is applied to the images for dimensionality reduction. Then, the lower dimensional image is divided into spectrally uniform regions using the quadtree approach. The third step is to extract the endmembers from each tile (quadrant) using SVDSS. Finally, abundances are computed using the methods shown in Section 2.4.3.

\subsubsection{Results of Urban Data Set}

First, superpixels were extracted using the SLIC algorithm, here we used the region (superpixel) size to be $16 \times 16$, this particular size was selected based on the work by [62]. The result was $20 \times 20$ grid of superpixels (total of 394 superpixels, some superpixels were very small, so they were merged with larger ones) and the image is shown in Figure 5.3(a). It is clear that the SLIC image is an over-segmented version of the original data image.

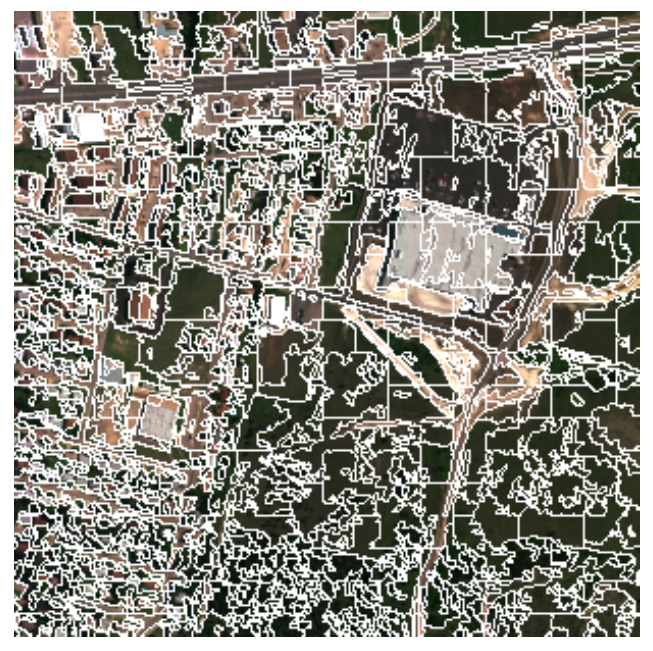

(a)

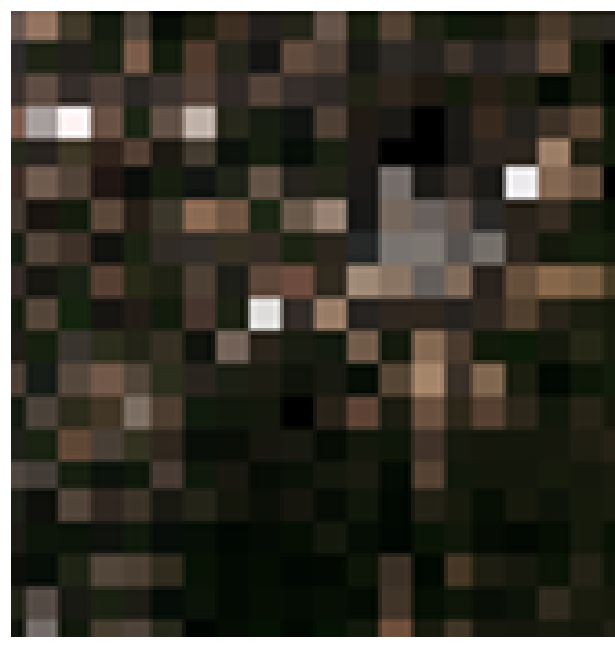

(b)

Figure 5.3: SLIC image of the urban scene: (a) SLIC over-segmentation; (b) low dimensional representation, each pixel replace by the mean.

For each superpixel, the spectral mean $(\mu)$ of the spectral signatures is calculated. Though the SLIC image has a non-uniform arrangement of superpixels, each superpixel initial start point was on a grid formed by the spectral means of each tile (see Section 2.5.1). We can assume that each 
superpixel has a certain coordinate on a non-uniform grid that will be represented by the spectral mean of the pixels in that superpixel.

The result is an image cube of size $20 \times 20 \times 162$ shown in Figure 5.3(b). The cube is partitioned using the quadtree method, the result of the partitioning is shown in Figure 5.4.

\begin{tabular}{|c|c|c|}
\hline $\begin{array}{r}\text { L } 1.1 \\
5 \times 5 \\
6 \text { EMs } \\
\end{array}$ & $\begin{array}{r}\text { L } 1.2 \\
5 \times 5 \\
6 \text { EMs } \\
\end{array}$ & \multirow{2}{*}{$\begin{array}{c}\mathrm{L} 2 \\
10 \times 10 \\
8 \mathrm{EMs}\end{array}$} \\
\hline $\begin{array}{c}\text { L } 1.3 \\
5 \times 5 \\
6 \text { EMs }\end{array}$ & $\begin{array}{c}\text { L } 1.4 \\
5 \times 5 \\
6 \text { EMs }\end{array}$ & \\
\hline \multicolumn{2}{|c|}{$\begin{array}{c}\text { L } 3 \\
10 \times 10 \\
7 \mathrm{EMs}\end{array}$} & $\begin{array}{c}\mathrm{L} 4 \\
10 \times 10 \\
6 \mathrm{EMs}\end{array}$ \\
\hline
\end{tabular}

Figure 5.4: Partitioning using quadtree (EMs = Endmembers).

To estimate the number of endmembers in each tile, the Gram matrix method was applied to each tile. The results are also shown in Figure 5.4.

Endmember extraction is performed on each tile using SVDSS, a total of 45 endmembers were extracted and shown in Figure 5.5. The extracted spectral endmembers were clustered using the cosine distance with a threshold of 0.005 . Clustering analysis resulted in 17 endmember spectral classes. Finally, abundance estimation using the non-negative sum less than one constraints (NNSLO) [52] was performed using the 45 endmembers over the whole image. The abundance for each class is the summation of abundances for the spectral endmembers belonging to the class. A post-processing step is to normalize the abundance maps to sum to one, by dividing the per pixel abundances by the sum of abundances. Figures 5.6 and 5.7 show the spectral endmember classes and abundances for the Urban scene.

In order to form the information classes, we merged spectral classes with abundances associated to information classes in the reference maps. Table 5.3 shows how the spectral endmember classes are related to the information classes of the Urban scene. Figures 5.8 and 5.9 show the 


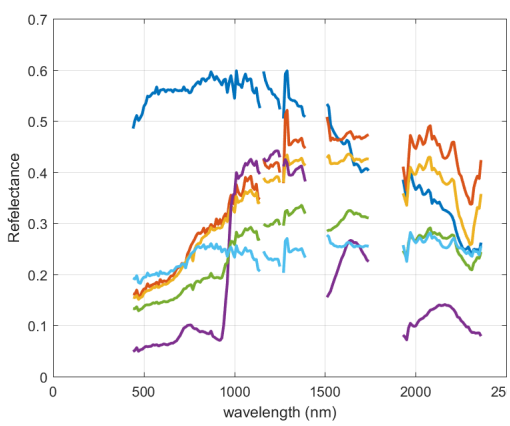

L 1.1

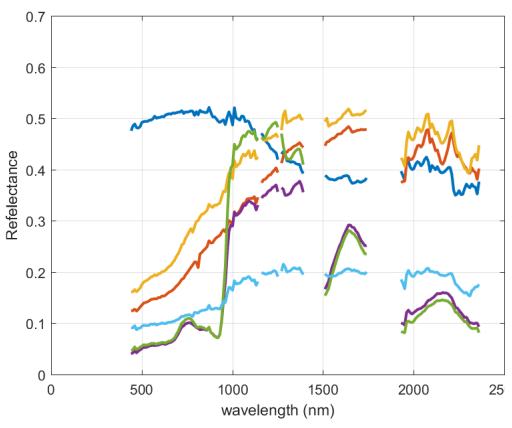

L 1.4

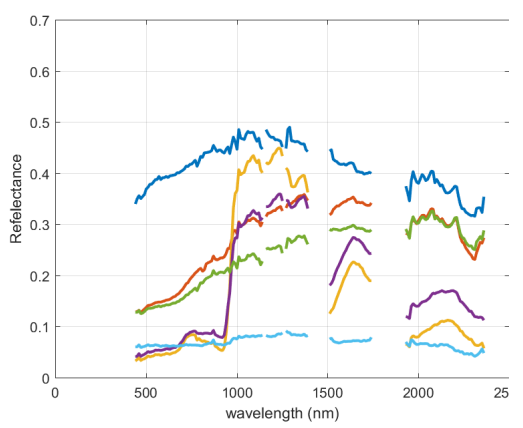

L 1.2

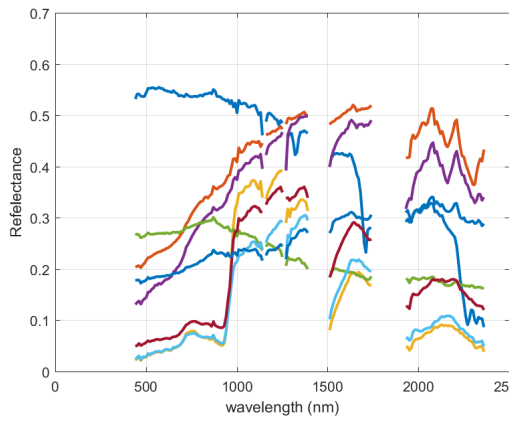

L 2

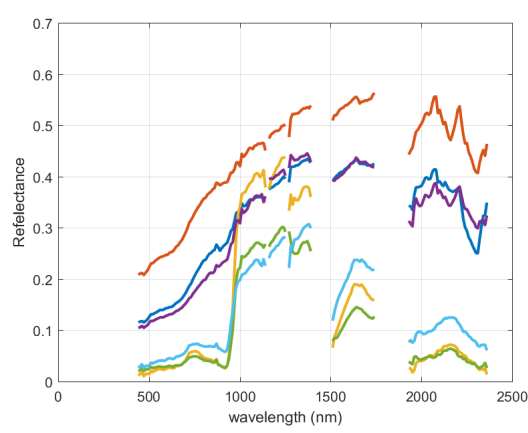

L 4

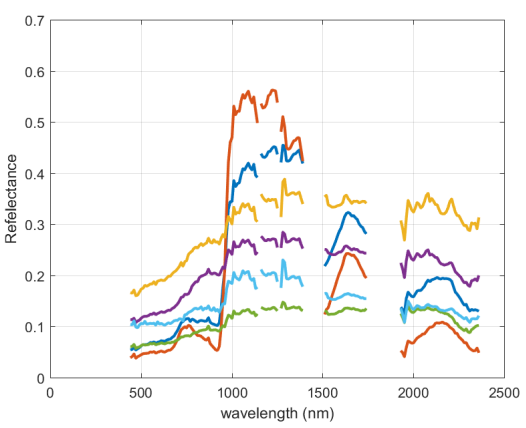

L 1.3

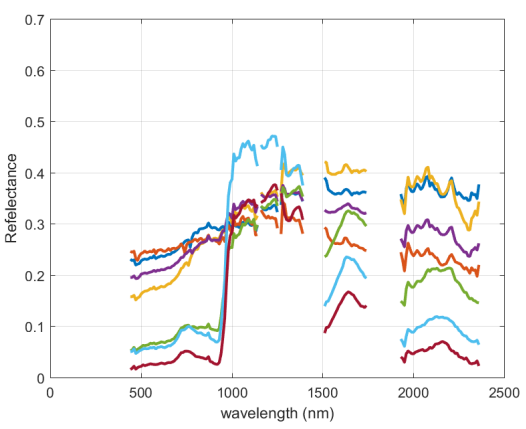

L 3

Figure 5.5: Endmembers for each tile.

information classes endmembers and their corresponding abundance maps respectively. The information class abundance maps are the sum of the endmembers classes abundances belonging to that information class. 


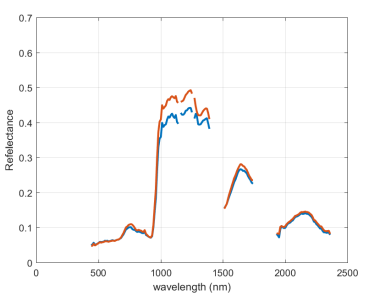

EC 1

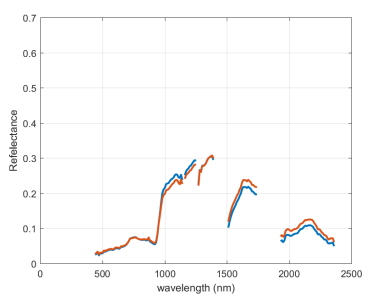

EC 4

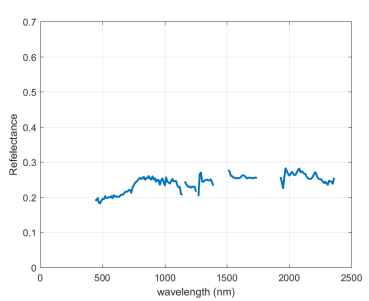

EC 7

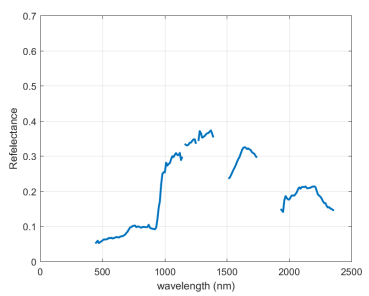

EC 10

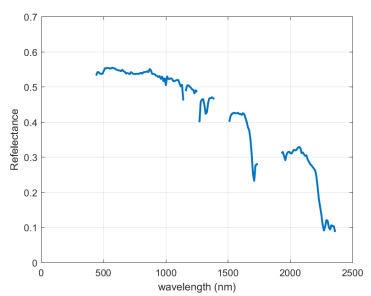

EC 13

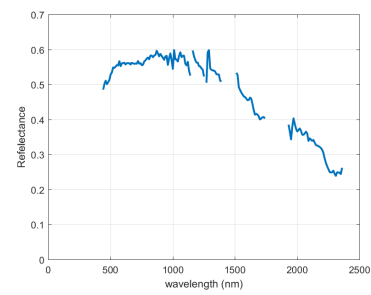

EC 2

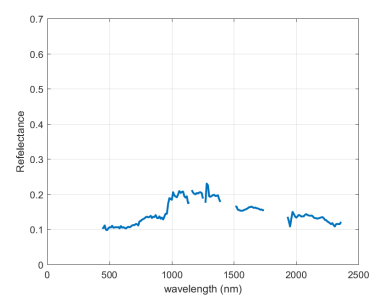

EC 5

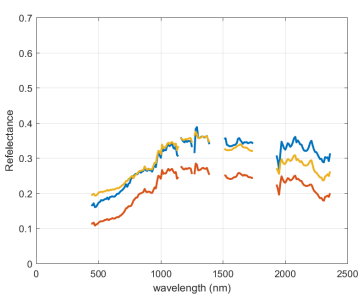

EC 8

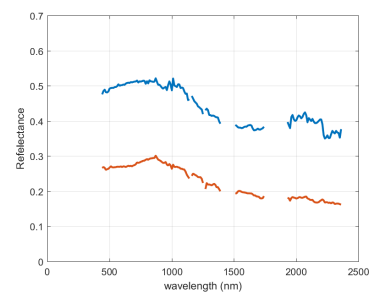

EC 11

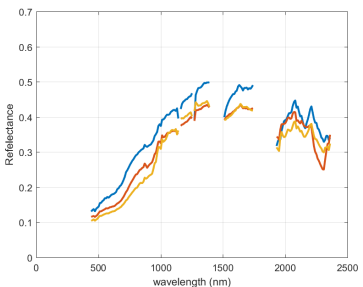

EC 14

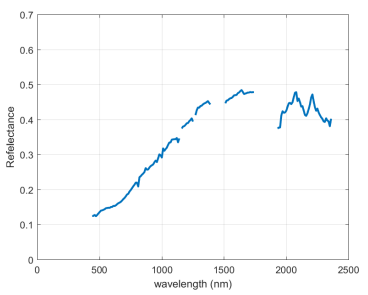

EC 3

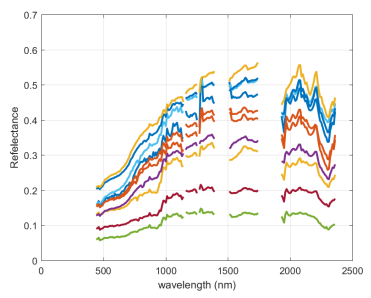

EC 6

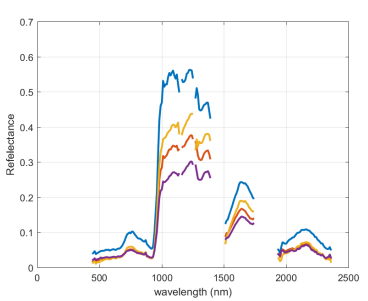

EC 9

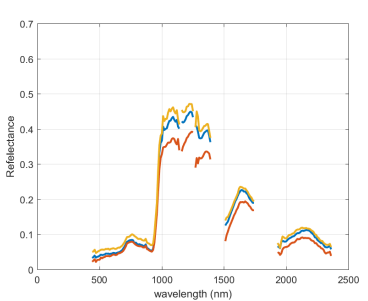

EC 12

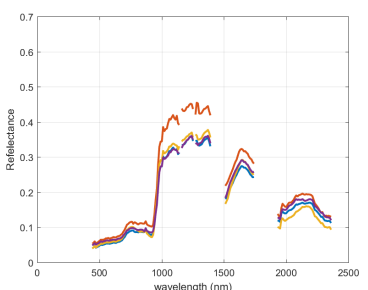

EC 15

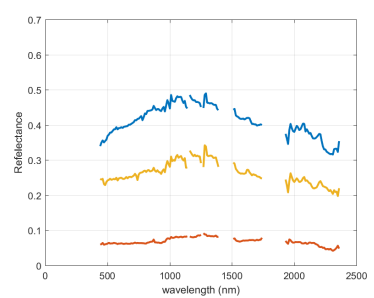

EC 16

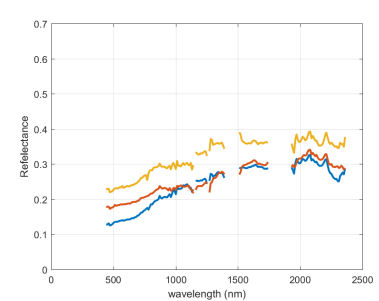

EC 17

Figure 5.6: Spectral endmembers classes for Urban using angle distance of 0.005 . 


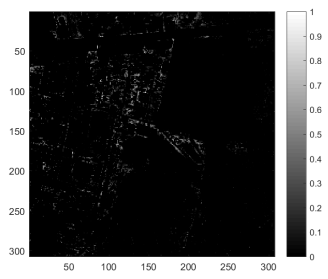

EC 1

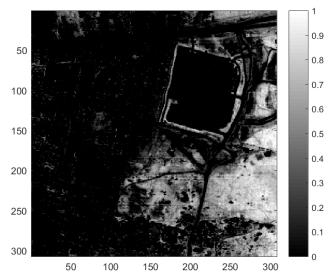

EC 4

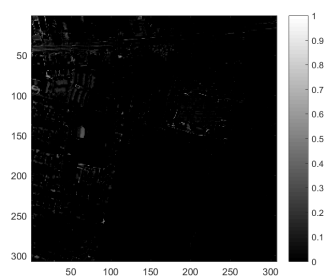

EC 7

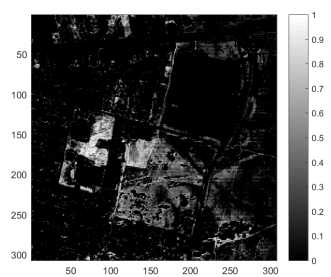

EC 10

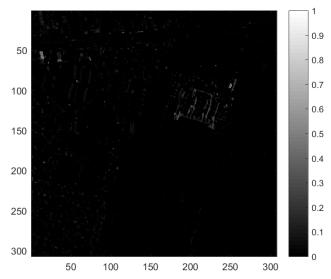

EC 13

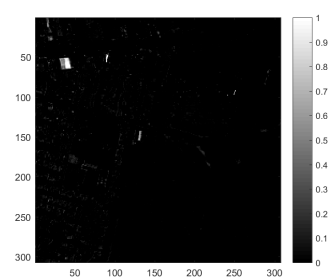

EC 2

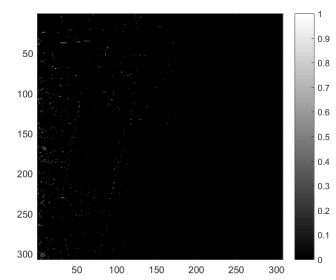

EC 5

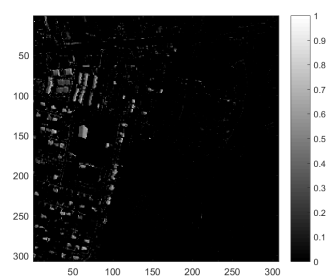

EC 8

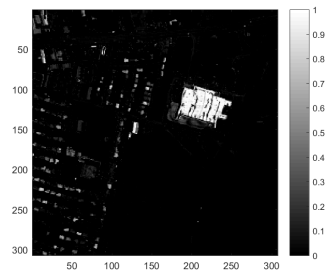

EC 11

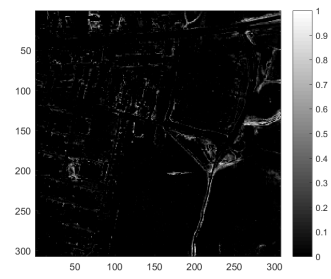

EC 14

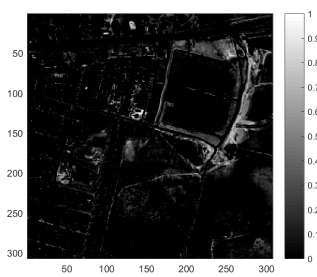

EC 3

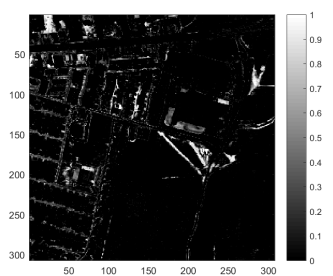

EC 6

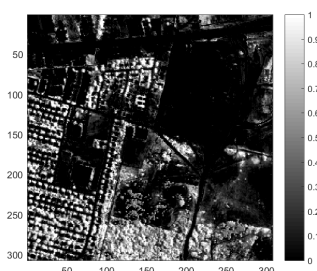

EC 9

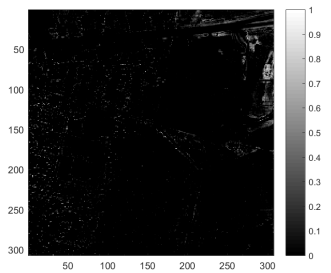

EC 12

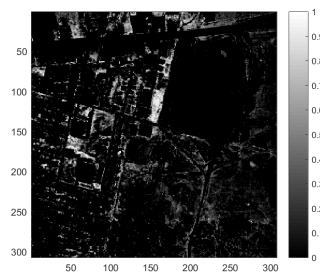

EC 15

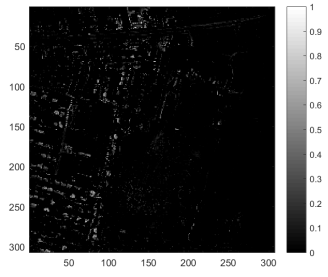

EC 16

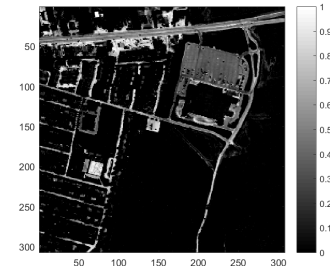

EC 17

Figure 5.7: Abundances of spectral endmember classes in Urban using proposed approach. 


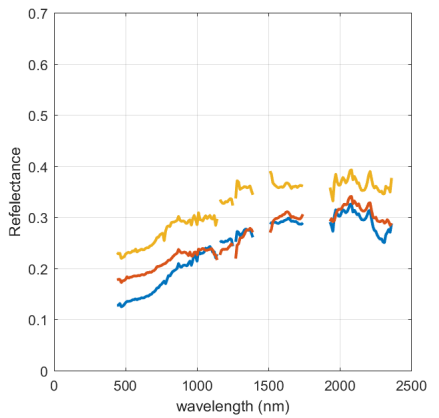

Road

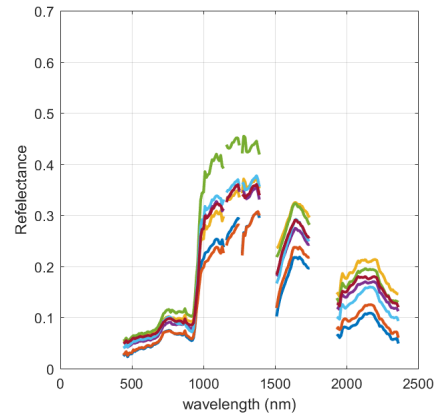

Grass

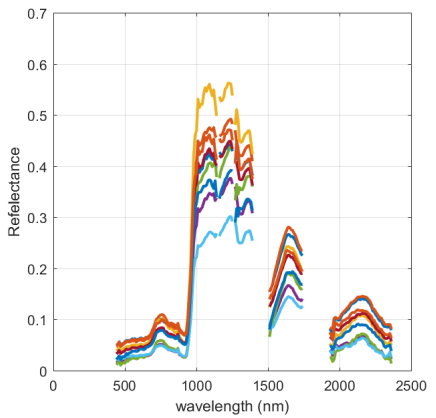

Trees
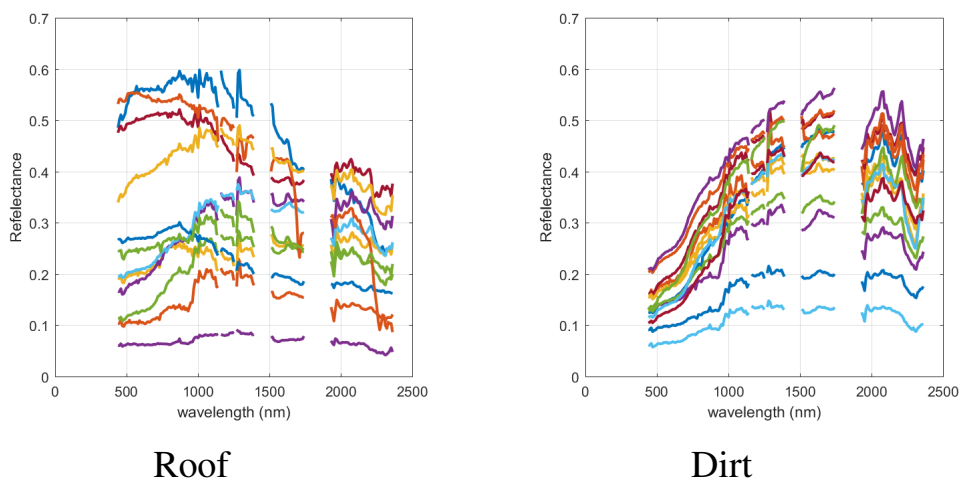

Figure 5.8: Resulting Urban information classes.

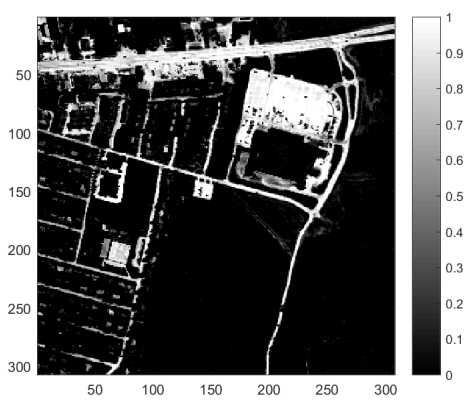

Road

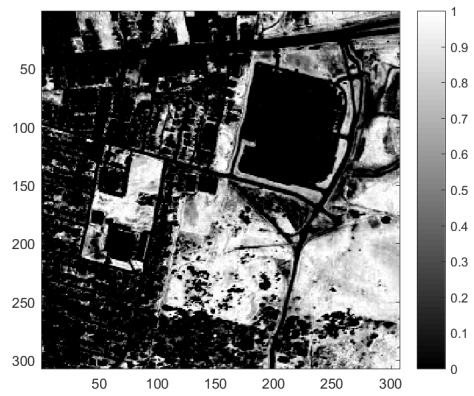

Grass

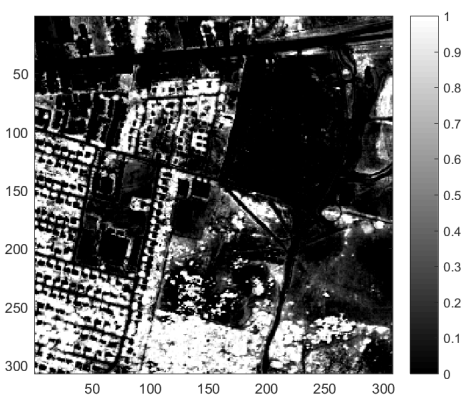

Trees

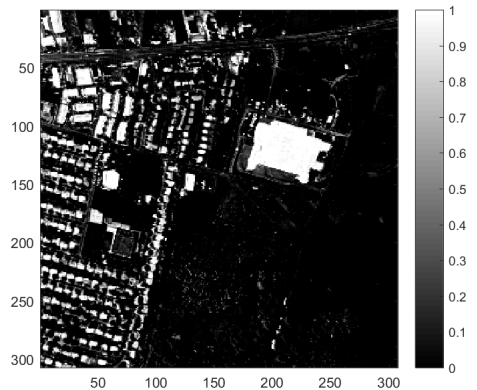

Roof

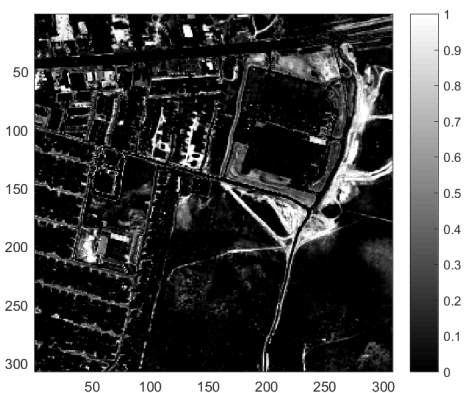

Dirt

Figure 5.9: Resulting Urban information classes abundance maps. 
Table 5.3: Relation between spectral endmember classes and information classes for Urban.

\begin{tabular}{|c|c|}
\hline Spectral Endmember Class & Information Class \\
\hline 17 & Road \\
\hline $4,10,15$ & Grass \\
\hline $1,9,12$ & Trees \\
\hline $2,5,7,8,11,13,16$ & Roof \\
\hline $3,6,14$ & Soil (Dirt) \\
\hline
\end{tabular}

\subsubsection{Qualitative Assessment of Unmixing Results for Urban scene}

Evaluation of unmixing results is performed using the assessment methodology presented in Section 5.3.1. The evaluation of unmixing results is performed by comparing extracted information endmember classes and estimated abundances with published spectral libraries and classification maps shown in Section 5.2.1.

Figure 5.10 presents a comparison between the information abundance maps extracted from the Urban scene using the proposed approach and the reference abundance maps obtained from [7]. A simple conclusion is that there is an agreement between the maps. Also, by looking at the error images (5.10 rightmost column), an easy observation is that the most of the error image is dark which indicates the the error values are small. However, by looking at Figure 5.12, when comparing the obtained endmembers for the roof class to the reference data, some of the spectral endmembers in the proposed method do not exactly match (in terms of shape) with the reference data. The same could be said for the Dirt class. This actually is not a problem and won't affect the analysis, because information classes describe a certain land cover and may contain multiple of spectral classes (Roof for example consist of 7 spectral classes $(2,5,7,8,11,13$ and 16) ). 


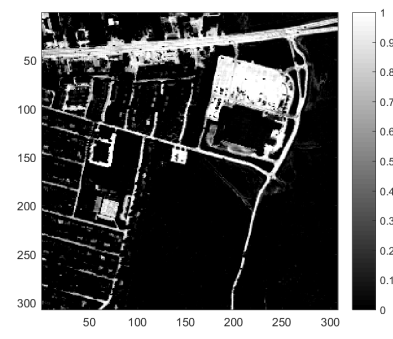

Road - Proposed Method

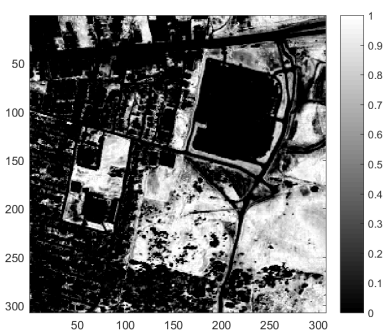

Grass - Proposed Method

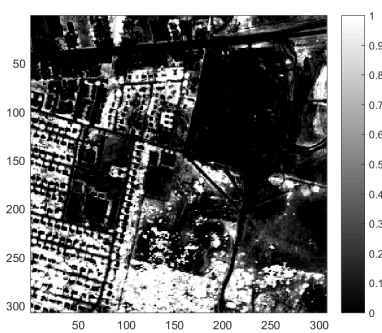

Trees - Proposed Method

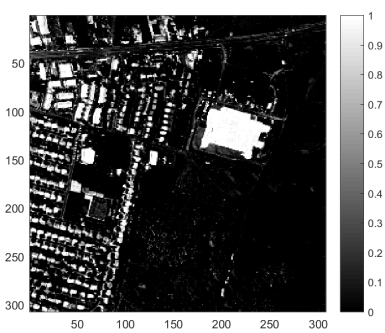

Roof - Proposed Method

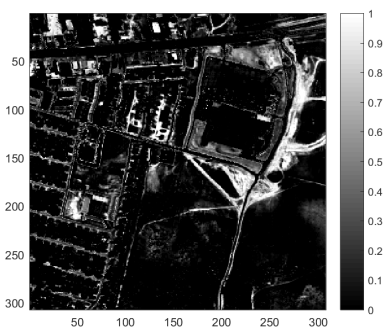

Dirt - Proposed Method

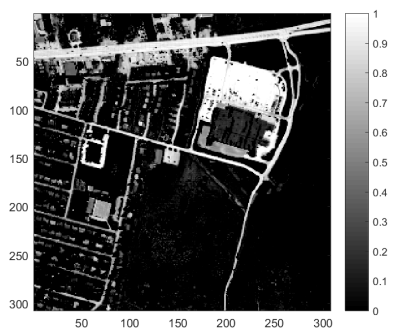

Road - Reference Data

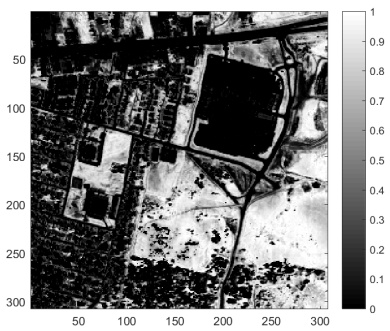

Grass - Reference Data

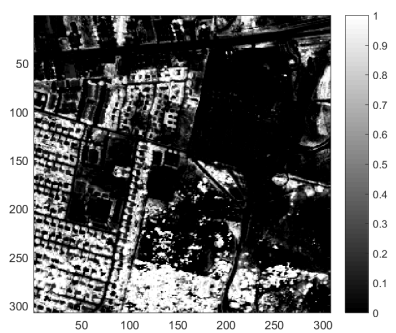

Trees - Reference Data

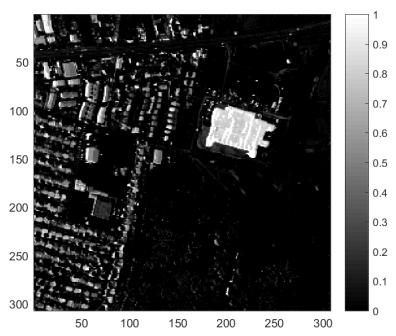

Roof - Reference Data

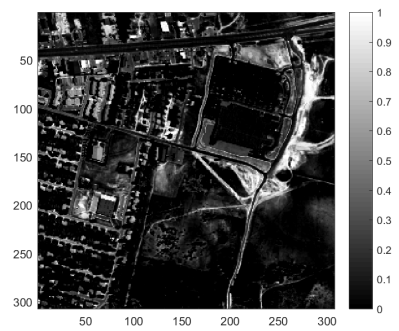

Dirt - Reference Data

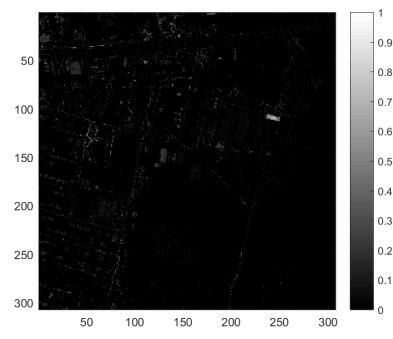

Road - Error Image

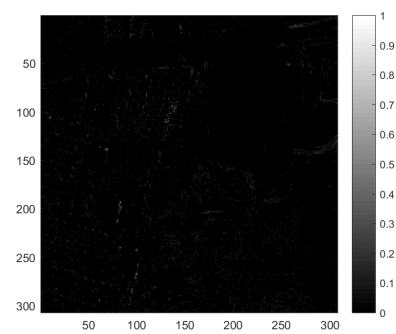

Grass - Error Image

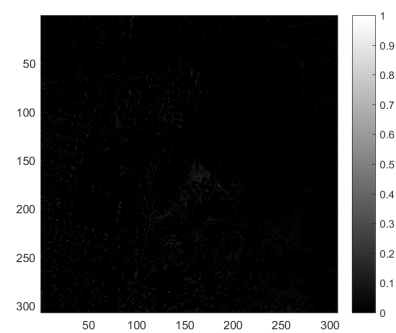

Trees - Error Image

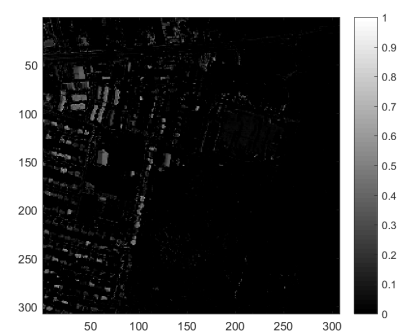

Roof - Error Image

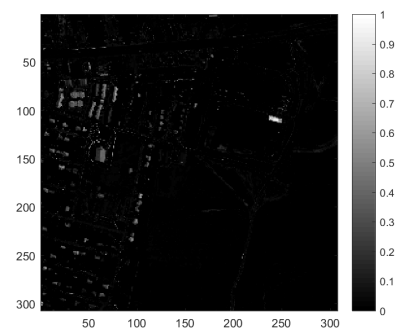

Dirt - Error Image

Figure 5.10: Comparison between abundance maps extracted using the proposed method and the reference data from [7]. 


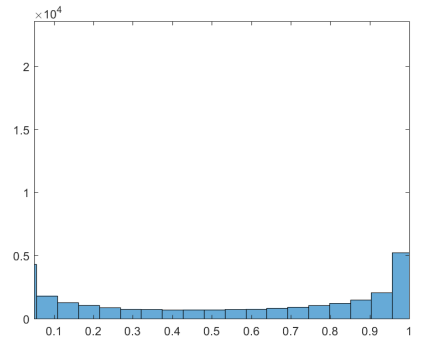

Road - Proposed Method

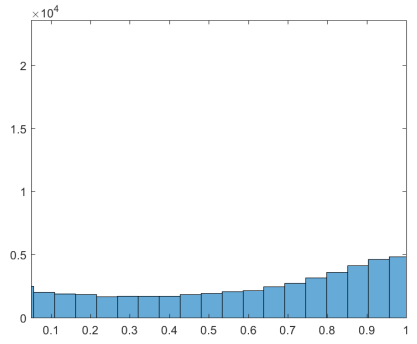

Grass - Proposed Method

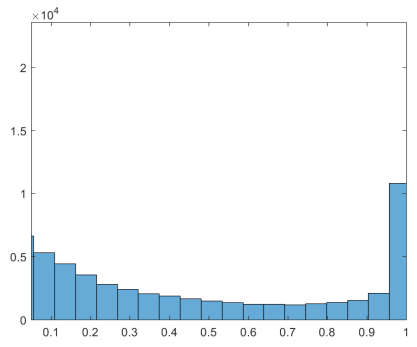

Trees - Proposed Method

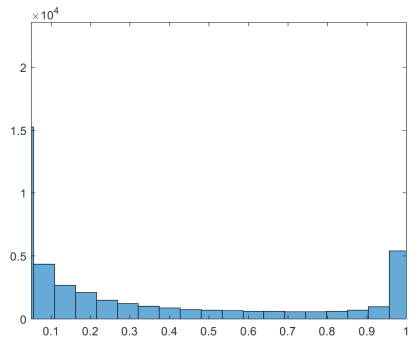

Roof - Proposed Method

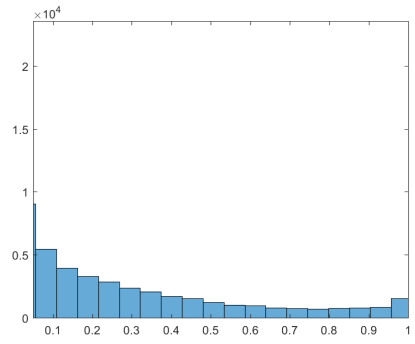

Dirt - Proposed Method

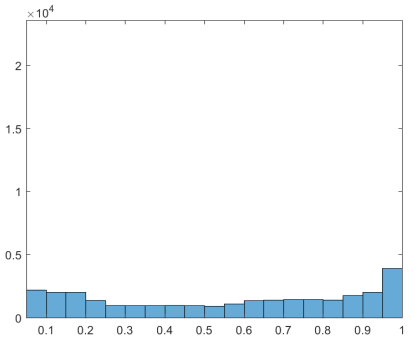

Road - Reference Data

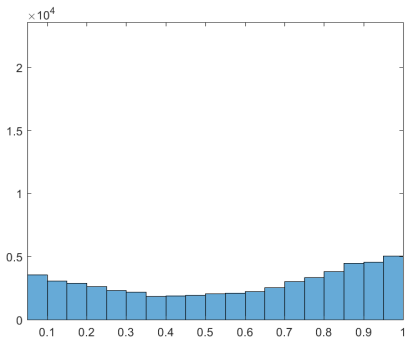

Grass - Reference Data

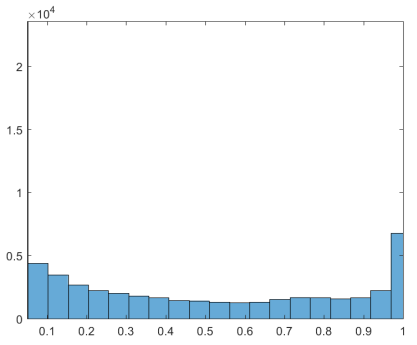

Trees - Reference Data

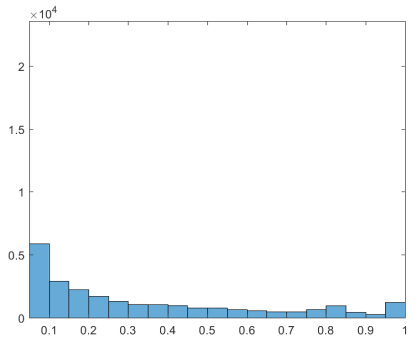

Roof - Reference Data

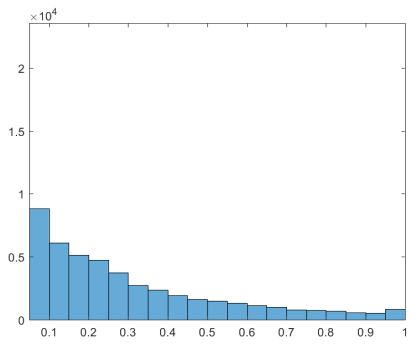

Dirt - Reference Data

\begin{tabular}{|l|c|}
\hline Maximum & 0.7485 \\
\hline Minimum & 0 \\
\hline Mean & 0.0117 \\
\hline
\end{tabular}

Road - Error statistics

\begin{tabular}{|l|c|}
\hline Maximum & 0.4719 \\
\hline Minimum & 0 \\
\hline Mean & 0.0102 \\
\hline
\end{tabular}

Grass - Error statistics

\begin{tabular}{|l|c|}
\hline Maximum & 0.2374 \\
\hline Minimum & 0 \\
\hline Mean & 0.0082 \\
\hline
\end{tabular}

Trees - Error statistics

\begin{tabular}{|l|c|}
\hline Maximum & 0.6784 \\
\hline Minimum & 0 \\
\hline Mean & 0.0196 \\
\hline
\end{tabular}

Roof - Error statistics

\begin{tabular}{|l|c|}
\hline Maximum & 0.9813 \\
\hline Minimum & 0 \\
\hline Mean & 0.0148 \\
\hline
\end{tabular}

Dirt - Error statistics

Figure 5.11: Histograms for the abundance maps in Figure 5.10 and error statistics. Pixels with value of zero were not included in the histogram plots as in most images they were the vast majority of pixels. 


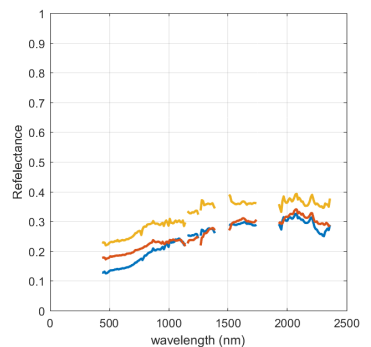

Road - Proposed Method

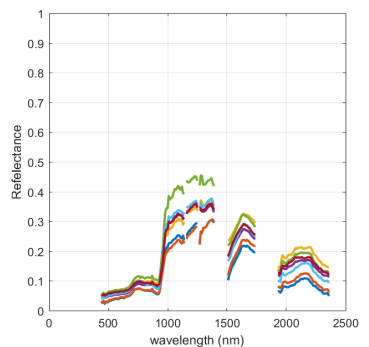

Grass - Proposed Method

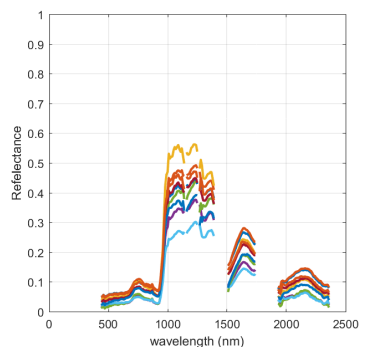

Trees - Proposed Method

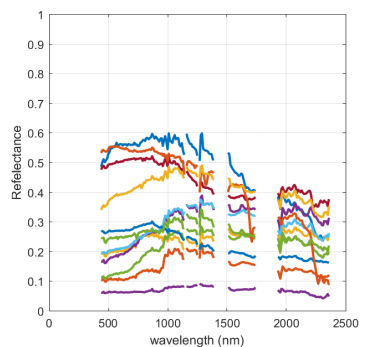

Roof - Proposed Method

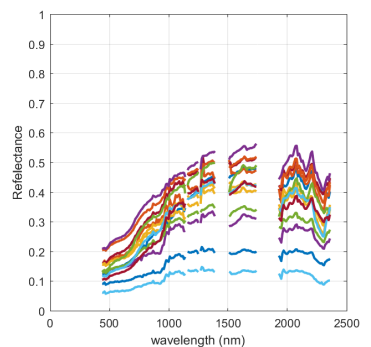

Dirt - Proposed Method

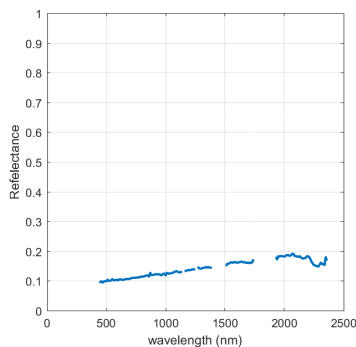

Road - Reference Data

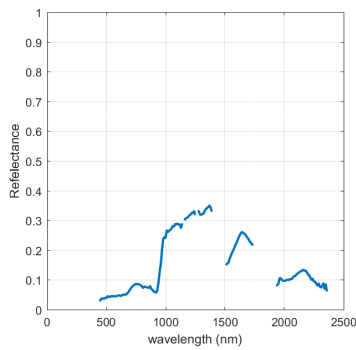

Grass - Reference Data

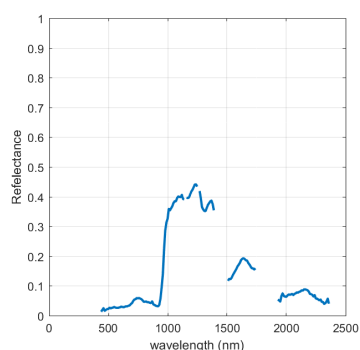

Trees - Reference Data

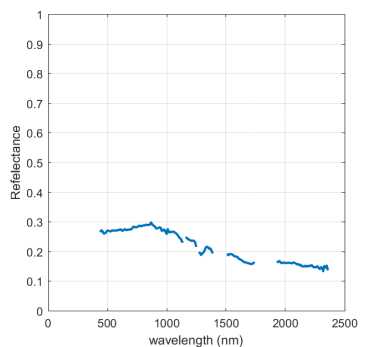

Roof - Reference Data

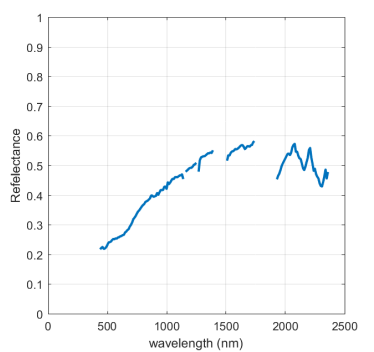

Dirt - Reference Data

Figure 5.12: Comparison of endmember classes extracted with the proposed algorithm and reference data spectral signatures for endmembers. 


\subsubsection{Quantitative Assessment of Unmixing Results of Urban Scene}

Before performing the analysis described in Section 5.3.2, some statistical analysis on the error image (Figure 5.10, rightmost column) were performed to give an indication of the performance of the algorithm: Maximum Error, Minimum Error and Mean Error. These statistics, alongside with the histogram of each abundance map, are shown in Figure 5.11. The mean of the error in all of the materials is small (less than 2\%) which indicates that the obtained abundance maps are similar to the reference data abundance maps. Figure 5.10 confirms the similarity of both maps.

The Histograms in Figure 5.11 give an idea about how pixels brightness value distribution and their recurrence. Zero pixels were excluded from the histogram representation since the comparison is in between the nonzero values of the abundance maps. The histograms look similar to each other. The Road class abundance distribution of the proposed approach look very similar to the one obtained from the reference data. Similar conclusion can be said for Trees and Grass classes. this is also confirmed by looking at Figure 5.10 where the abundance maps are similar and the error image is almost dark (very small errors). The Roof and Dirt histograms do not look as identical as the first three materials. Again, this is confirmed at the error image of Figure 5.10 where the error maps has some bright pixels.

For quantitative analysis, a classification map is generated using the estimated abundances and the relation of spectral endmember classes and information classes (Table 5.3). The resulting classification maps and the reference maps are compared using the confusion matrix. Four statistics are computed: Producer's Accuracy, User's Accuracy, Overall Accuracy and the Cohen's Kappa [87].

We will then perform a post-processing step to the resulting class map to clean it from scattered individual pixels (known as salt and pepper noise), the assumption here is that information classes will occupy relatively uniform spatial areas (larger than just few pixels). The clean-up here will be performed using a spatial median filter of size $3 \times 3$ to remove the scattered pixels and produce a smoother version of the class map. Similar analysis using the confusion matrix will be performed on the smoothed image. 
Figure 5.13 shows the classification maps for the Urban scene. Figure 5.13(a) shows the classification map generated from the abundance maps estimated by the proposed algorithm. Figure 5.13(b) shows the classification map from Figure 5.13(a) after being smoothed with a median filter. Figure 5.13(c) shows the reference data classification map. Table 5.4 presents the Confusion matrix for the original case. Table 5.5 shows the confusion matrix of the smoothed image.

The classification map obtained from the unmixing results has an overall agreement of $84.64 \%$ with the reference map. All Classes have a producer's accuracy higher than $80 \%$. This high agreement can be also noted in Figure 5.13. Dirt has the lowest producer's accuracy (80.26\%). 871 Dirt pixels were classified as Roof and this can be seen in the Figure, the upper left area (row number 100 and column number 5) showed that some pixels were labeled as Roof while they should be Dirt. Many pixels that belong to the Trees class in the reference map were classified as Roof class with 2108 pixels, this is due the intimate mixing between the two classes, and it can be seen by looking at the left region in Figure 5.13.

In the end, the final assessment was the Cohen's Kappa score which was $79.40 \%$ which indicates a Substantial agreement based on Table 5.2.

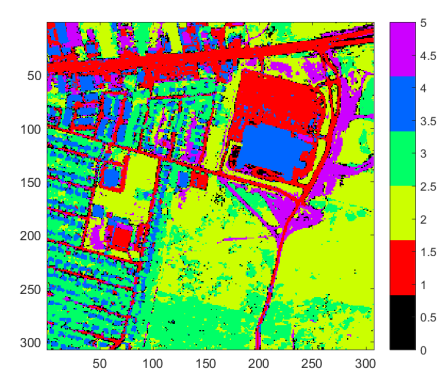

(a)

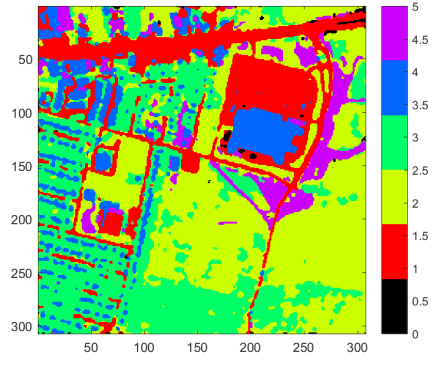

(b)

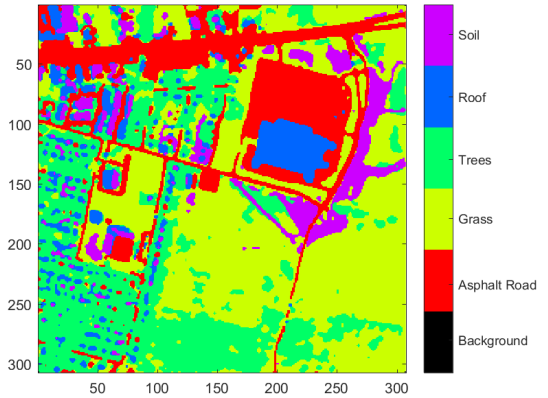

(c)

Figure 5.13: Urban Image: (a) Classification map using the abundances and the majority vote criterion; (b) Classification map after filtering; (c) Reference data classification map [7]

Similar evaluation analysis is performed on the median filtered class map. Here, the class map of Figure 5.13(a) is passed through a spatial median filter to remove the scattered pixels in the image and produce a more uniform map, the result of the filtering is shown in Figure 5.13(b), Table 5.5 shows the confusion matrix between the filtered class map and the reference data class map. An 
Table 5.4: Confusion matrix between reference map and generated classification map from unmixing results.

\begin{tabular}{|c|c|c|c|c|c|c|c|c|}
\hline & \multicolumn{5}{|c|}{ Reference Data } & \multirow[b]{2}{*}{ Totals } & \\
\hline & & Road & Grass & Trees & Roof & Dirt & & $\begin{array}{c}\text { User's } \\
\text { Accuracy (\%) }\end{array}$ \\
\hline \multirow{7}{*}{$\begin{array}{l}\text { Class } \\
\text { Map }\end{array}$} & Road & 13775 & 296 & 368 & 96 & 238 & 14773 & 93.24 \\
\hline & Grass & 44 & 29443 & 1162 & 34 & 223 & 30906 & 95.27 \\
\hline & Trees & 59 & 2633 & 20068 & 188 & 31 & 22979 & 87.33 \\
\hline & Roof & 1569 & 400 & 2108 & 6106 & 871 & 11054 & 55.24 \\
\hline & Dirt & 717 & 1542 & 736 & 281 & 5543 & 8819 & 62.85 \\
\hline & Totals & 16164 & 34314 & 24442 & 6705 & 6906 & 88531 & \\
\hline & $\begin{array}{c}\text { Producer's } \\
\text { Accuracy (\%) }\end{array}$ & 85.22 & 85.80 & 82.10 & 91.07 & 80.26 & & $\begin{array}{c}\text { Overall } \\
\text { Accuracy }=84.64 \%\end{array}$ \\
\hline
\end{tabular}

Table 5.5: Confusion matrix between reference map and filtered classification map from unmixing results.

\begin{tabular}{|c|c|c|c|c|c|c|c|c|}
\hline & \multicolumn{5}{|c|}{ Reference Data } & \multirow[b]{2}{*}{ Totals } & \\
\hline & & Road & Grass & Trees & Roof & Dirt & & $\begin{array}{c}\text { User's } \\
\text { Accuracy }(\%)\end{array}$ \\
\hline \multirow{7}{*}{$\begin{array}{l}\text { Class } \\
\text { Map }\end{array}$} & Road & 15369 & 1187 & 758 & 88 & 345 & 17747 & 86.6 \\
\hline & Grass & 248 & 32484 & 458 & 20 & 156 & 33366 & 97.36 \\
\hline & Trees & 442 & 1968 & 23678 & 362 & 145 & 26595 & 89.03 \\
\hline & Roof & 1055 & 257 & 1082 & 6387 & 1086 & 9867 & 64.73 \\
\hline & Dirt & 169 & 365 & 85 & 69 & 5510 & 6198 & 88.90 \\
\hline & Totals & 17283 & 36261 & 26061 & 6926 & 7242 & 93773 & \\
\hline & $\begin{array}{c}\text { Producer's } \\
\text { Accuracy (\%) }\end{array}$ & 88.93 & 89.58 & 90.86 & 92.22 & 76.08 & & $\begin{array}{c}\text { Overall } \\
\text { Accuracy } 88.97 \%=\end{array}$ \\
\hline
\end{tabular}

improvement to the user's accuracy for most of the classes is observed. This shows that filtering of the class map made a better representation and easier to compare with the reference data. Also, the overall accuracy increased to be $88.97 \%$. To better evaluate this approach, the kappa coefficient was calculated and the value was $85.01 \%$ which indicates an almost perfect agreement with the reference data.

\subsubsection{Results for Pavia University Data Set}

Similar approach is followed to analyze the Pavia University data set, the image is clustered using SLIC using a region size of $16 \times 16$, the result was $39 \times 22$ grid of superpixels as shown in Figure 
5.14(a).

The superpixels spectral means are shown in 5.14(b) to form a low dimensional image cube

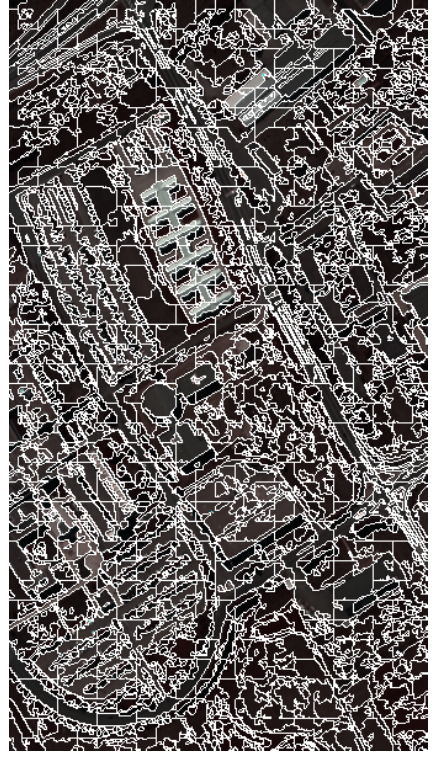

(a)

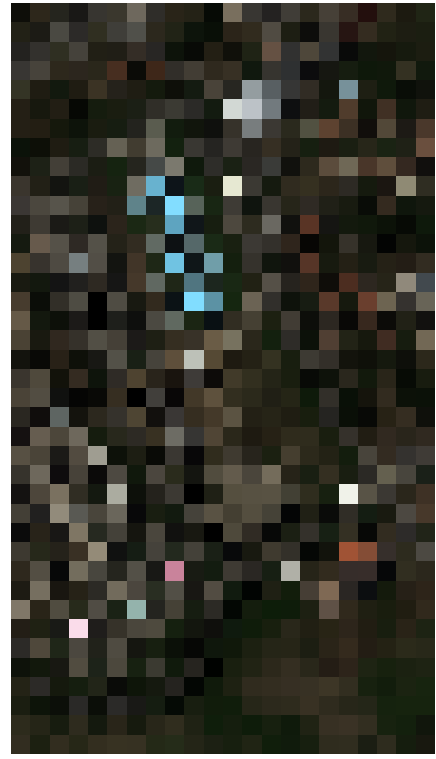

(b)

Figure 5.14: (a) SLIC image of the Pavia data set; (b) RGB display of the low dimensional superpixel image

of size $39 \times 22 \times 103$. The LD cube is partitioned using the quadtree approach as shown in Figure 5.15, which also shows the estimated number of endmembers using the Gram method.

Endmember extraction is performed on each tile using SVDSS (see Figure 5.16 for results). A total of 58 endmembers were extracted. The extracted spectral endmembers were clustered using the cosine distance with a threshold of 0.005 . Clustering analysis resulted in 20 spectral classes. Finally, abundance estimation using the non-negative sum to or less than one constraints (NNSLO) [52] was performed using the 58 endmembers over the whole image. The abundance for each class is the sum of abundances for the spectral endmembers belonging to the class. A post-processing step is to normalize the abundance maps to one as discussed previously.

Figures 5.17 and 5.18 show the spectral classes and their corresponding abundance maps for the Pavia scene.

After obtaining the spectral classes, information classes are created based on the types of land cover of the scene. An information class might contain one or many spectral classes. By looking at 


\begin{tabular}{|c|c|c|c|}
\hline & & $\begin{array}{c}\text { L } 2.1 \\
9 \times 5 \\
6 \mathrm{EMs}\end{array}$ & $\begin{array}{c}\mathrm{L} 2.2 \\
9 \times 5 \\
6 \mathrm{EMs}\end{array}$ \\
\hline $6 \mathrm{H}$ & Ms & L 2.3 & L 2.4 \\
\hline L 3.1 & L 3.2 & & \\
\hline $9 \times 5$ & $9 \times 5$ & & \\
\hline 5 EMs & $6 \mathrm{EMs}$ & & 4 \\
\hline L 3.3 & L 3.4 & & Ms \\
\hline $9 \times 5$ & $9 \times 5$ & & \\
\hline 5 EMs & 6 EMs & & \\
\hline
\end{tabular}

Figure 5.15: Pavia Partitioning using quadtree (EMs = Endmembers)

Table 5.6: Relation between Spectral classes and information classes for Pavia scene

\begin{tabular}{|c|c|}
\hline Spectral Endmember Class & Information Class \\
\hline $1,2,3,4,5,16,20$ & Asphalt + Bitumen \\
\hline 18 & Meadows \\
\hline $9,13,15$ & Gravel + Brick \\
\hline 6,11 & Trees \\
\hline 12 & Metal Sheet \\
\hline $7,8,19$ & Bare Soil \\
\hline 10,14 & Shadow \\
\hline
\end{tabular}

Figure 5.19, an easy observation is that Gravel and Brick classes have similar spectra, and similarly to Asphalt and Bitumen classes. So, we decided to combine both Gravel and Brick to form a single information class, and Asphalt with Bitumen into another information class. Table 5.6 shows the spectral classes and their contribution to the information classes. Figure 5.21 and 5.22 show the information classes endmembers and their corresponding abundance maps respectively.

By further studying the scene, comparing the RGB image with the class map (Figure 5.2), some regions were classified as Meadows yet they look more like soil (see Figure 5.20). Also by comparing the spectral signatures for these two classes, a similarity can be observed between them. It is suggested that both of the classes will be merged together to form a single information class. 


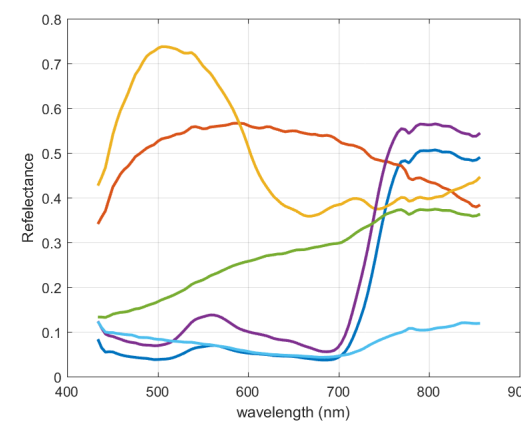

L 1

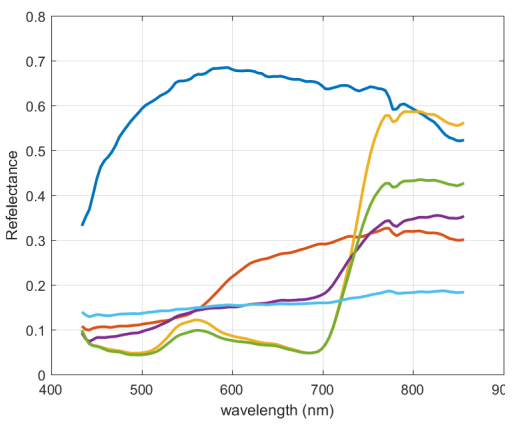

L 2.3

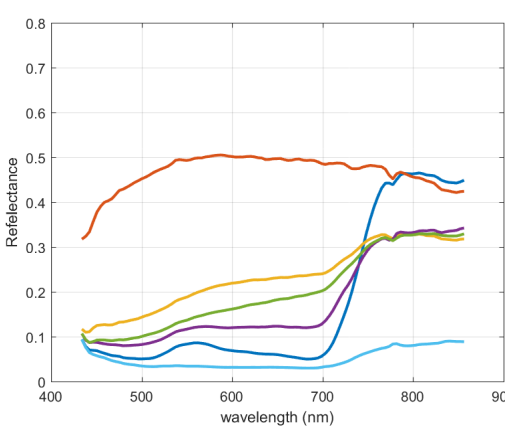

L 3.2

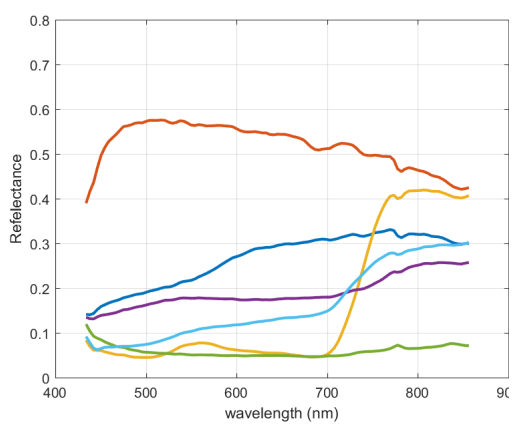

L 2.1

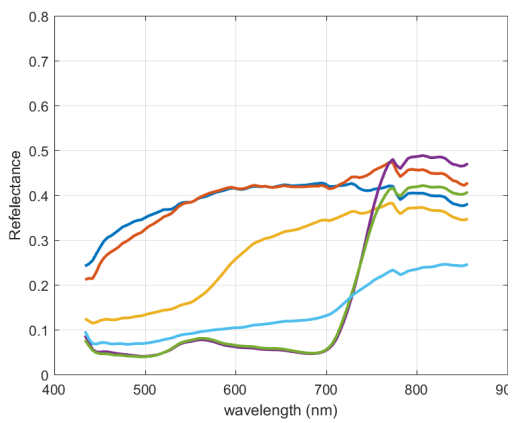

L 2.4

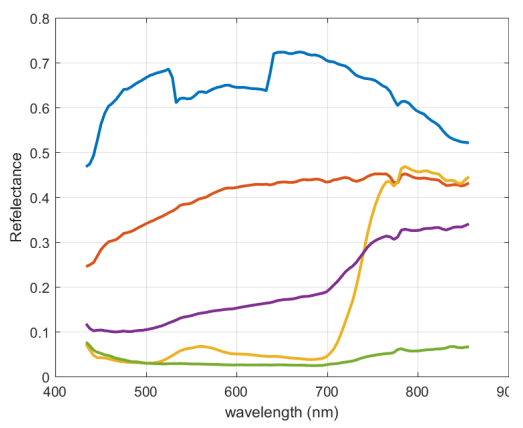

L 3.3

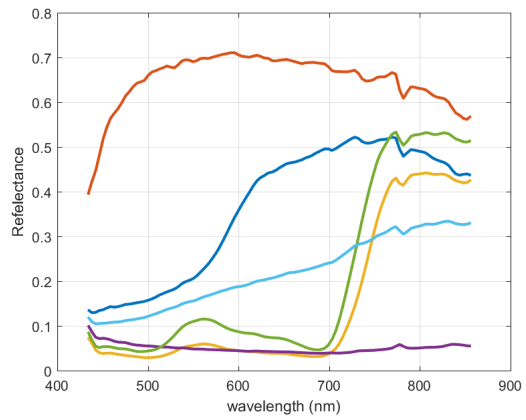

L 4

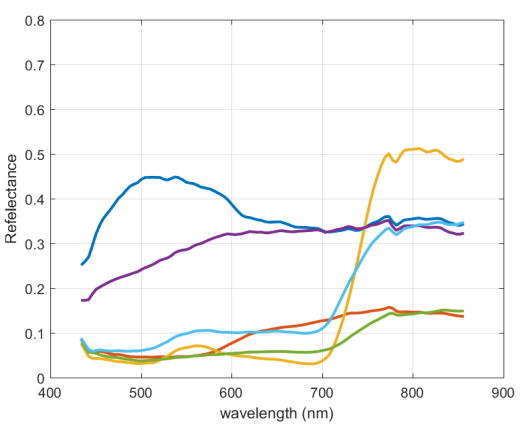

L 2.2

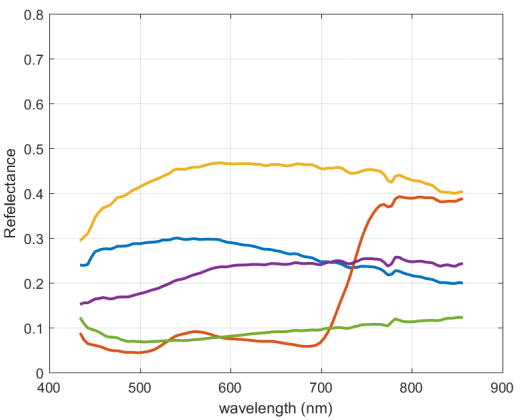

L 3.1

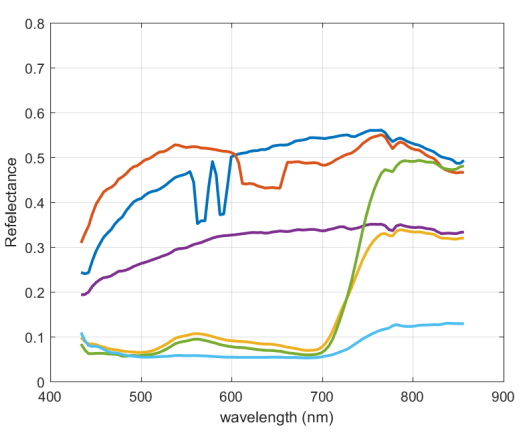

L 3.4

Figure 5.16: Endmembers for each tile. 

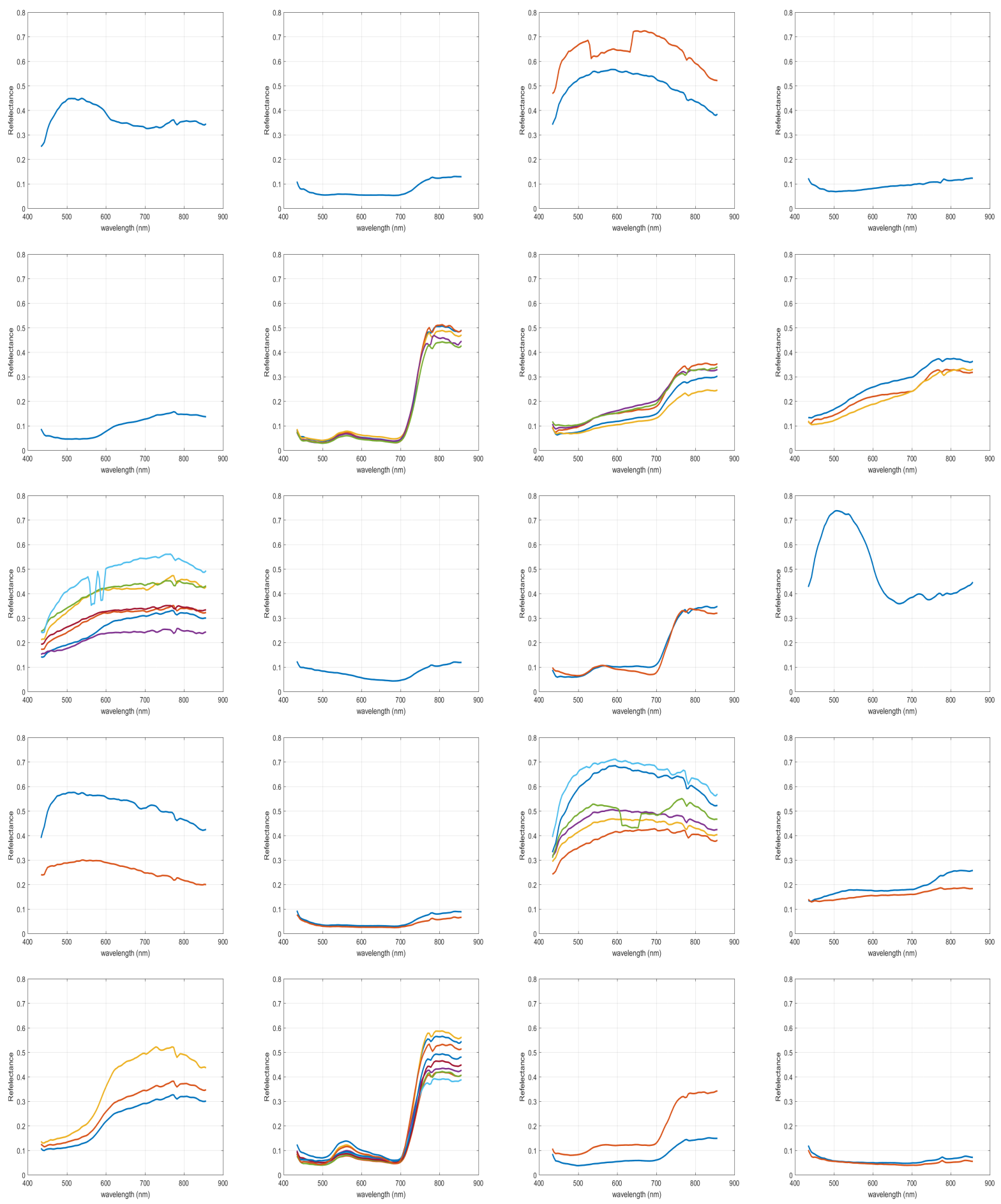

Figure 5.17: Spectral endmembers classes for Pavia scene using angle distance of 0.005 .

\subsubsection{Qualitative Assessment of Unmixing Results for Pavia Scene}

Evaluation of unmixing results is performed using the assessment methodology presented in Section 5.3.1. The evaluation of unmixing results is performed by comparing extracted information 

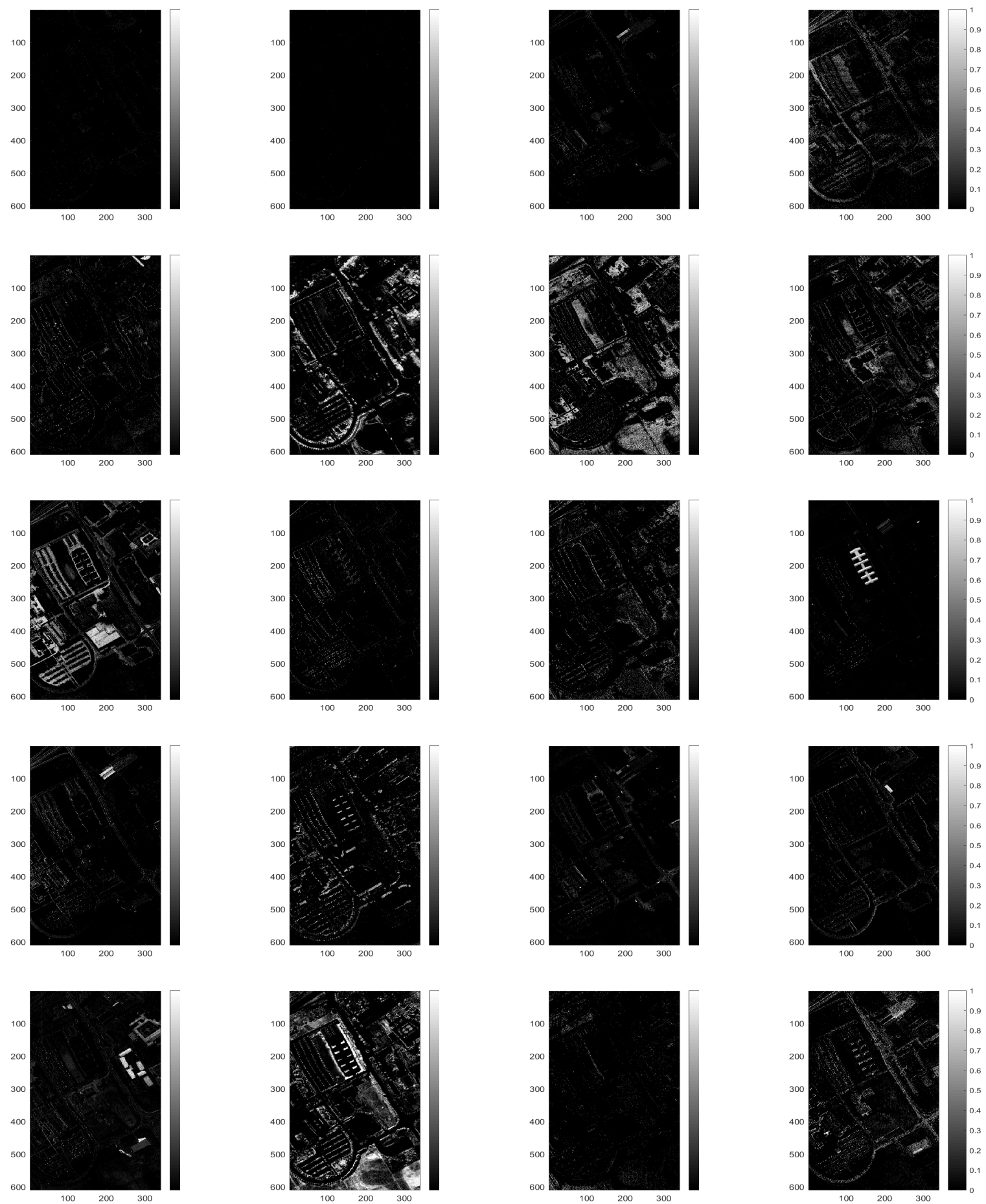

Figure 5.18: Spectral endmembers classes for Pavia scene using angle distance of 0.005 .

endmember classes and estimated abundances with published spectral libraries and classification maps shown in Section 5.2.2.

The comparison will be based on the reference data of the scene. However, no information 


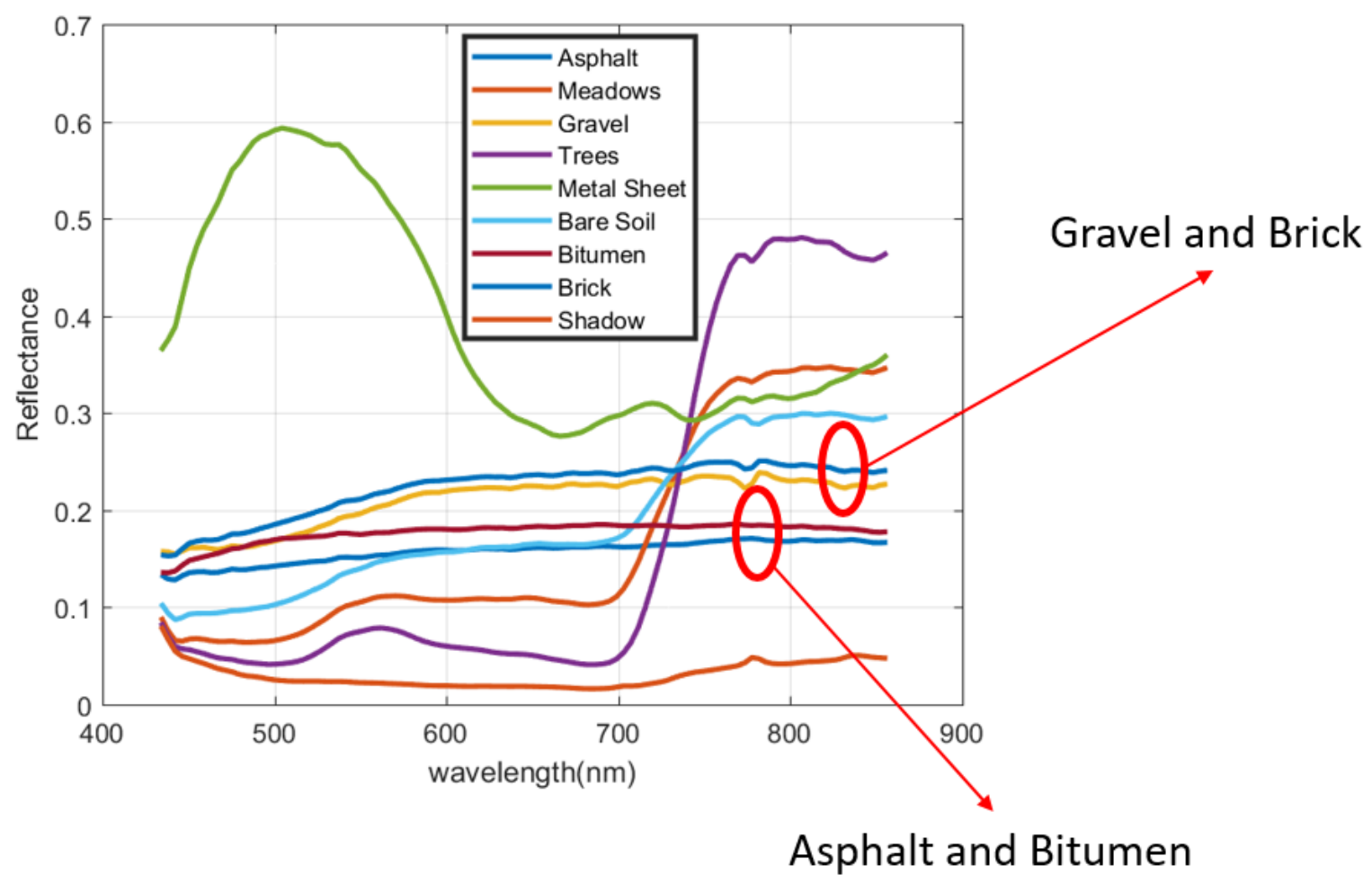

Figure 5.19: combining similar spectra of the Pavia Scene.

regarding the abundance maps is available, so the comparison will be based on the similarity of extracted abundances and the reference data class maps. Figure 5.23 shows a comparison between the extracted abundance map for the Asphalt and Bitumen classes with the available reference data. It is noticed that the class map is contained in the abundance map. A part of the road in the abundance map does not appear in the class map. By looking at Figure 5.2(b), that part was considered as background in the reference map. Our approach successfully captures the structure of the road (we can confirm this by visually inspecting the RGB image of the scene in Figure 5.2(a)).

Figure 5.24 shows the results of the second class (Meadows and Bare Soil), a lot of agreement is there between the reference data class map and the resulting abundance map. Similar conclusion is obtained by doing the same comparison between the other four classes (See Figure 5.25) 


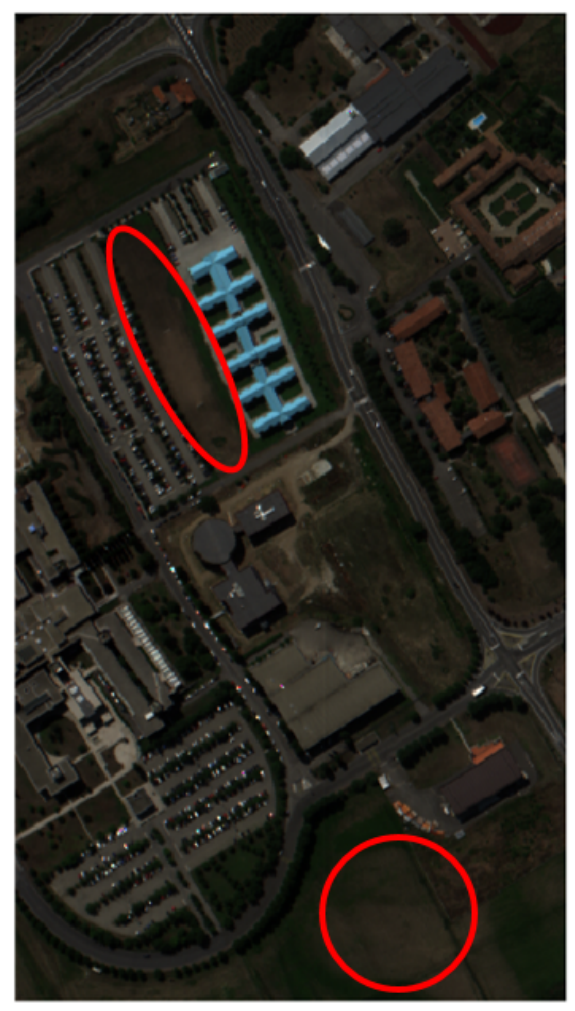

Figure 5.20: Pavia Image

\subsubsection{Quantitative Assessment of Unmixing Results of Pavia Scene}

Due to the lack of valid abundance maps form the reference data, error analysis could not be performed. The focus will be here on constructing the class map from the obtained abundance maps. As in Section 5.4.1.2, the resulting map will be compared to the reference map through the confusion matrix. The same four statistics are used to evaluate the agreement with reference data.

Figure 5.26 shows the classification maps for the Pavia scene. Figure 5.26(a) shows the classification map generated from the abundance maps estimated by the proposed algorithm. Figure 5.26(b) shows the classification map after smoothing with a median filter. Figure 5.26(c) shows the reference data classification map after merging similar spectral classes. To compute the confusion matrix, the comparison did not include pixels labeled as background in the reference data. Table 5.7 presents the Confusion matrix for Figure 5.26(a), while Table 5.8 shows the confusion matrix of Figure 5.26(b).

The classification map obtained from the unmixing results has an overall agreement of $90.24 \%$ 


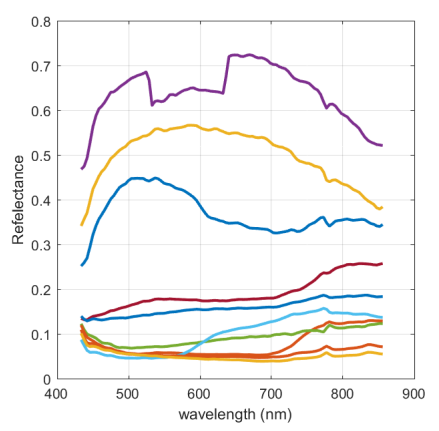

Asphalt and Bitumen

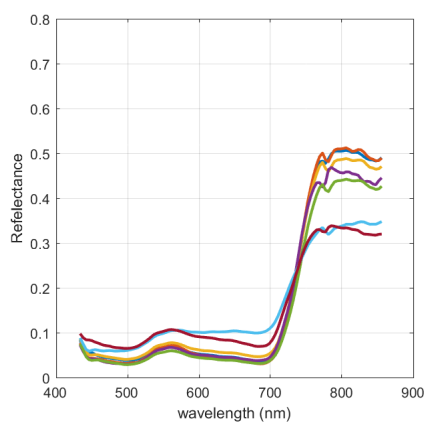

Trees

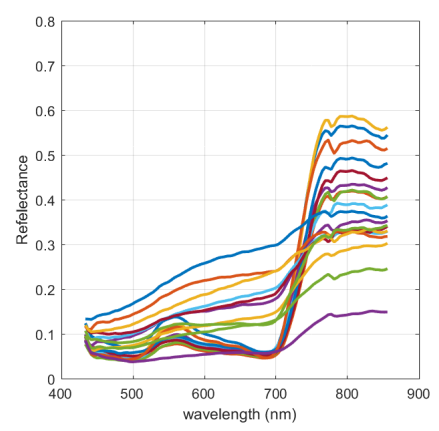

Meadows + Bare Soil

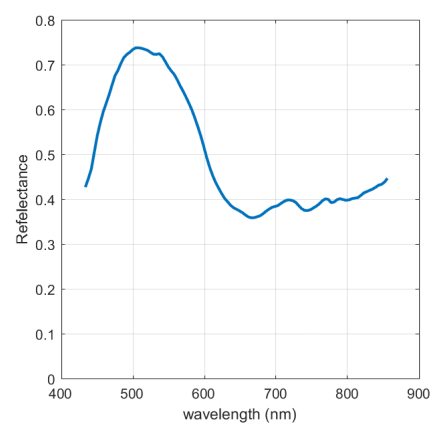

Metal Sheet

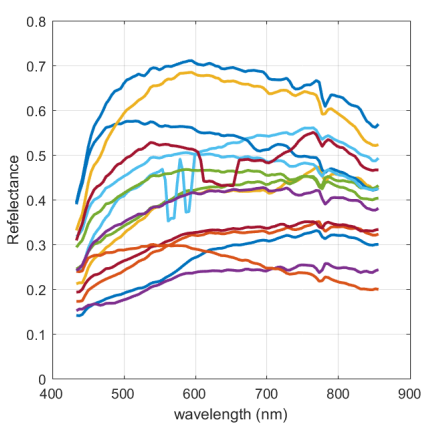

Gravel and Brick

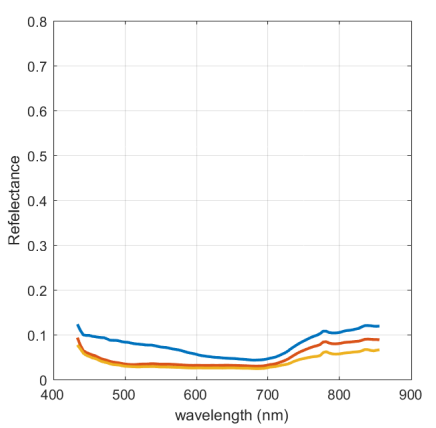

Shadows

Figure 5.21: Results Pavia information classes Signatures.

(Table 5.7). The producer's accuracy for the merged classes is high (over 89\%) which means that there is a high agreement between the generated class map with the the reference data. This is due to the large spatial contribution of the three classes in the image. The Metal sheet class has a very high producer's accuracy. This is due to two factors: first, the spectral signature of this class is unique and it is easily separated using clustering techniques. The second factor is that this material is hardly mixed with any other land-cover type, and occupies a relatively uniform connected region in the scene. The Trees class has a relatively low producer's accuracy (almost 66\%). Many of this class pixels were classified as Meadows and Soil class. The reason is obvious since the spectral signature of this class is very similar to the Meadows class and also that the spatial distribution of the Trees class in not uniform (most of the class pixels are scattered in the image and has an intimate mixing with other classes). The Shadows class has a moderate producer's accuracy due to mixing with the Asphalt and Bitumen class. The reason for such mixing is the similarity in spectral shape between these classes as shown in Figure 5.21. The magnitudes are of different scale, the 


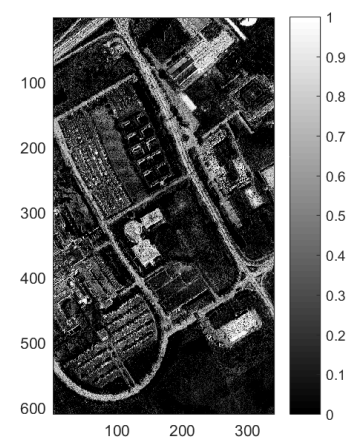

Asphalt and Bitumen

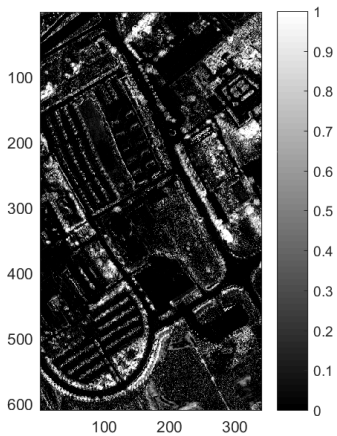

Trees

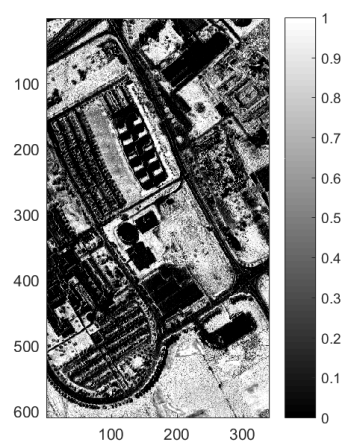

Meadows + Bare Soil

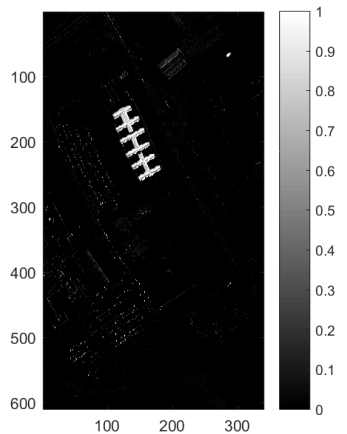

Metal Sheet

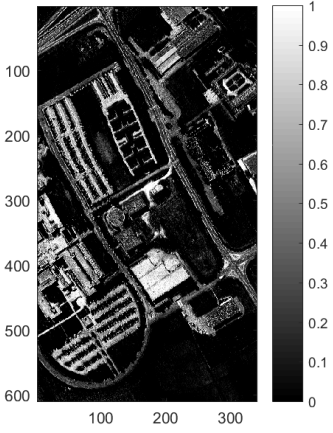

Gravel and Brick

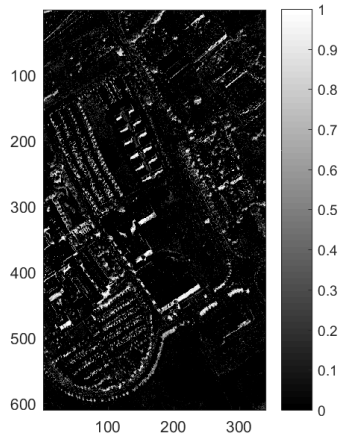

Shadows

Figure 5.22: Results Pavia information classes abundances.

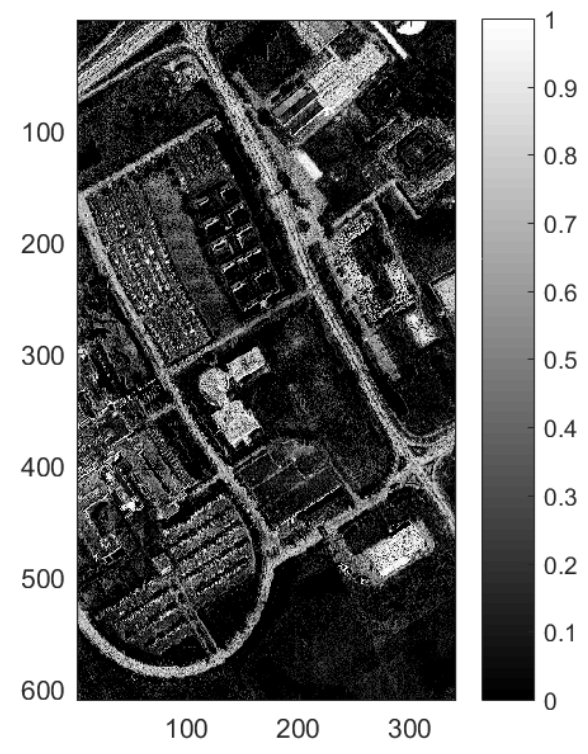

Proposed approach

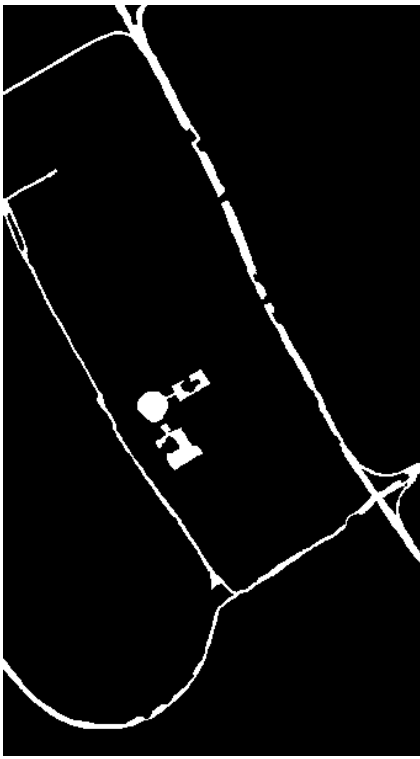

Class map (Reference data)

Figure 5.23: Comparison of Asphalt and Bitumen classes on Pavia set.

angle distance will cluster endmembers with similar shape regardless of magnitude. In the end, the Kappa coefficient for the confusion matrix is $83.77 \%$ which indicates an almost perfect agreement. 


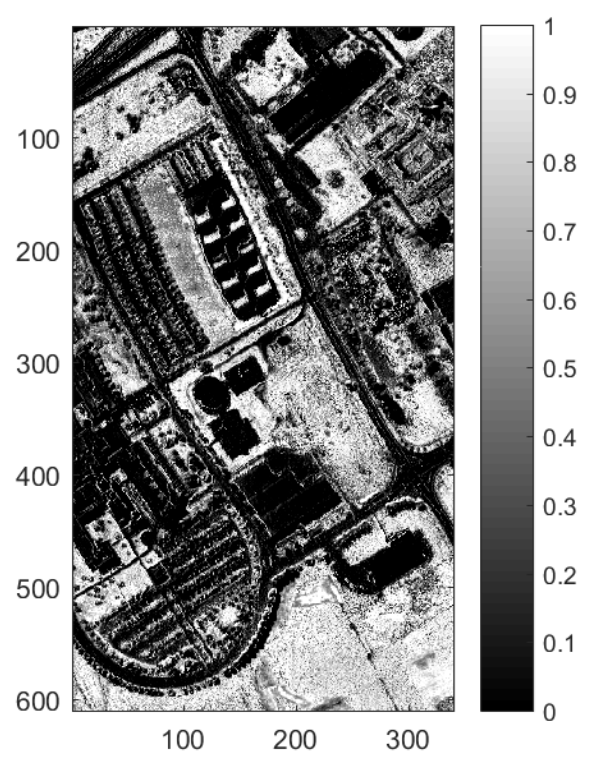

Proposed approach

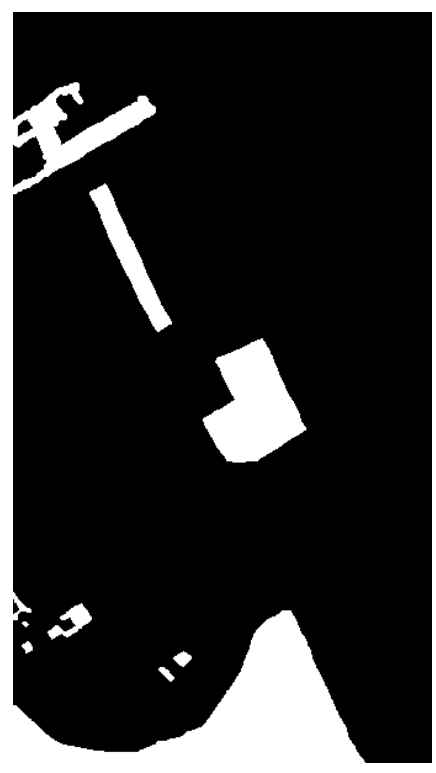

Class map (Reference data)

Figure 5.24: Comparison of Meadows and Bare Soil classes on Pavia set.

The classification map obtained from the unmixing after filtering has an overall agreement of 90.89\% (Table 5.8). This is a little bit higher than the original map. The producer's accuracy for the Asphalt and Bitumen class is $100 \%$. This is due to the median filter that eliminated scattered pixels. The Meadows and Soil class also showed a slight improvement with $95.41 \%$ accuracy. However, the filtering technique did not improve the results for the Gravel and Brick class. The reason behind this is, by looking at the spatial distribution for this particular class, that it does not have a large uniform spatial region to dominate the majority of pixels in the filter window. This may lead to drop some actual class pixels and be classified as another class. This conclusion is also applicable to the other classes. However, the Kappa coefficient for the confusion matrix of the filtered class map gives a value of $84.39 \%$ which is slightly better than the previous one.

\subsection{Comparing With Other methods}

This section presents comparative analysis of the proposed approach results with other existing approaches. We first compare with the results of global analysis where there is no use of spatial information. This methods is considered the classical way to extract endmembers from a scene. 


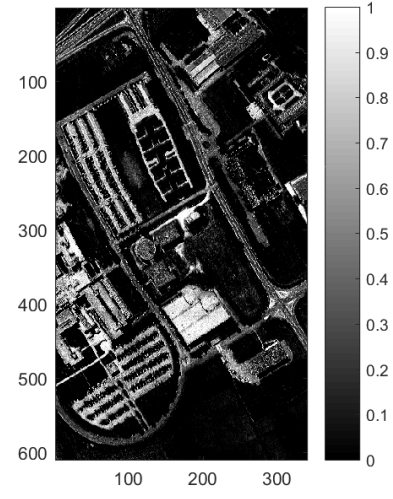

(a) Proposed approach (Gravel and Brick)

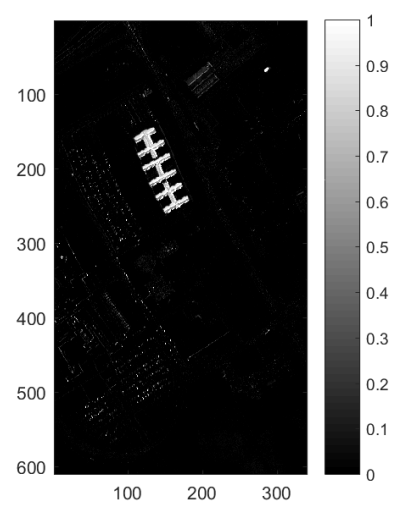

(e) Proposed approach (Metal Sheet)

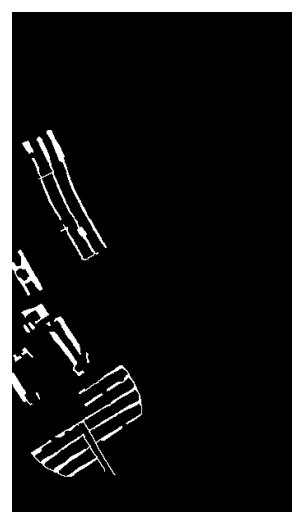

(b) Class map (Reference

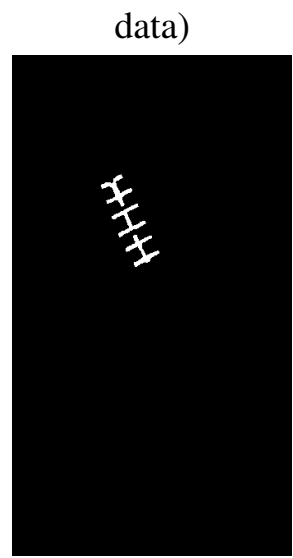

(f) Class map (Reference data)

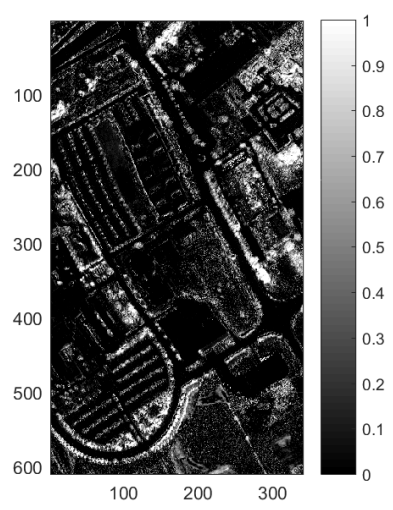

(c) Proposed approach (Trees)

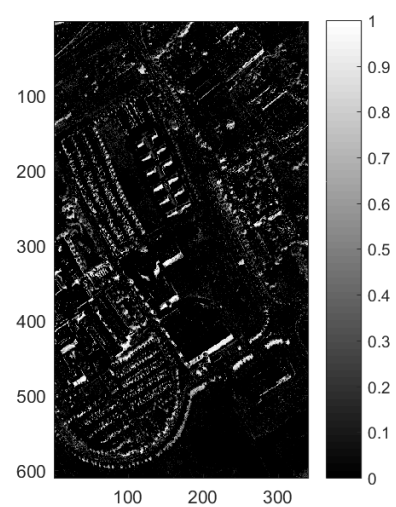

(g) Proposed approach (Shadows)

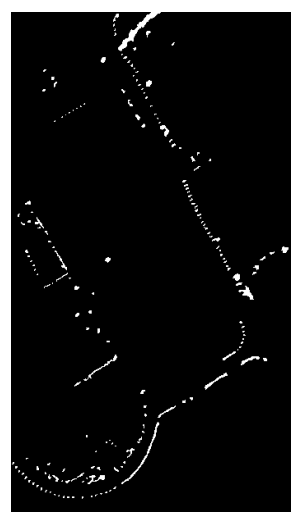

(d) Class map (Reference data)

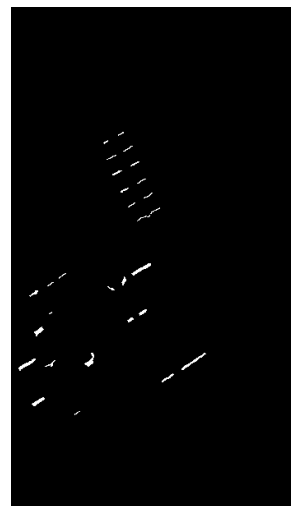

(h) Class map (Reference data)

Figure 5.25: Comparison of classes on Pavia set.

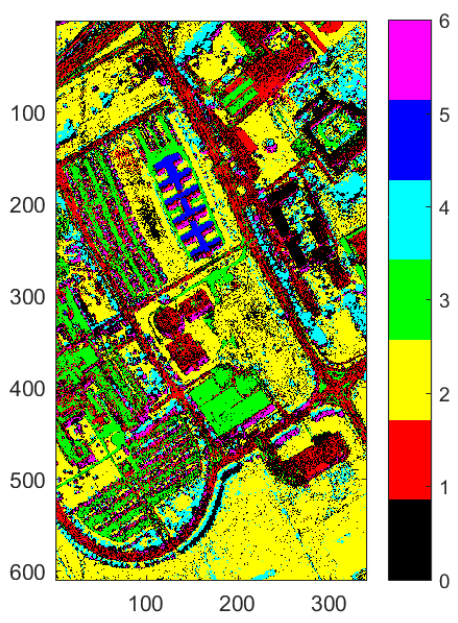

(a)

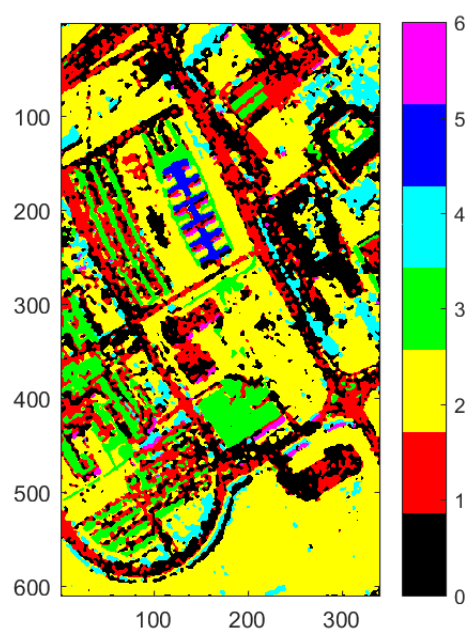

(b)

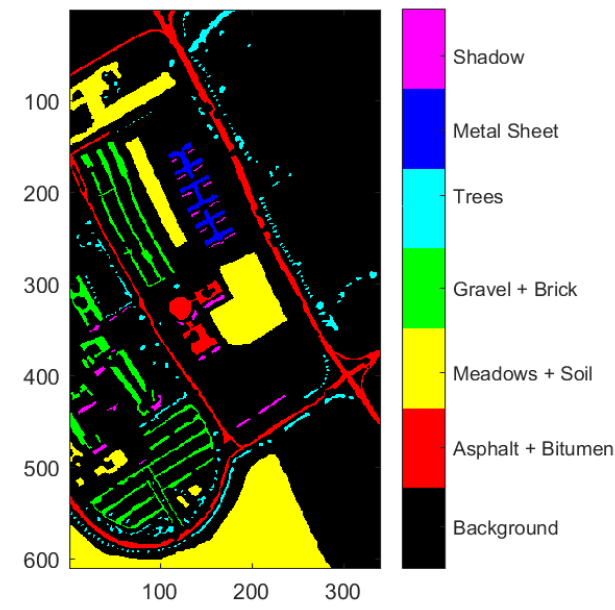

(c)

Figure 5.26: Pavia Image: (a) Classification map using the abundances and the majority vote criterion; (b) Classification map after filtering; (c) New reference data classification map. 
Table 5.7: Confusion matrix between reference map and generated classification map from unmixing results.

\begin{tabular}{|c|c|c|c|c|c|c|c|c|c|}
\hline & \multicolumn{6}{|c|}{ Reference Data } & \multirow[b]{2}{*}{ Totals } & \multirow[b]{2}{*}{$\begin{array}{c}\text { User's } \\
\text { Accuracy (\%) }\end{array}$} \\
\hline & & $\begin{array}{c}\text { Asphalt and } \\
\text { Bitumen }\end{array}$ & $\begin{array}{c}\text { Meadows } \\
\text { and Soil }\end{array}$ & $\begin{array}{c}\text { Gravel } \\
\text { and Brick }\end{array}$ & Trees & Metal Sheet & Shadow & & \\
\hline \multirow{8}{*}{$\begin{array}{l}\text { Class } \\
\text { Map }\end{array}$} & $\begin{array}{l}\text { Asphalt and } \\
\text { Bitumen }\end{array}$ & 3933 & 190 & 497 & 2 & 6 & 112 & 4740 & 82.97 \\
\hline & $\begin{array}{l}\text { Meadows } \\
\text { and Soil }\end{array}$ & 28 & 19012 & 16 & 925 & 2 & 0 & 19983 & 95.14 \\
\hline & $\begin{array}{c}\text { Gravel } \\
\text { and Brick }\end{array}$ & 282 & 536 & 4224 & 4 & 1 & 0 & 5047 & 83.69 \\
\hline & Trees & 0 & 683 & 0 & 1874 & 0 & 0 & 2557 & 73.29 \\
\hline & Metal Sheet & 1 & 0 & 0 & 0 & 1225 & 0 & 1226 & 99.84 \\
\hline & Shadow & 24 & 4 & 3 & 2 & 2 & 433 & 468 & 92.52 \\
\hline & Totals & 4268 & 20425 & 4740 & 2807 & 1236 & 545 & 34021 & \\
\hline & $\begin{array}{c}\text { Producer's } \\
\text { Accuracy (\%) }\end{array}$ & 92.15 & 93.08 & 89.11 & 66.74 & 99.11 & 79.45 & & $\begin{array}{c}\text { Overall } \\
\text { Accuracy }=90.24\end{array}$ \\
\hline
\end{tabular}

Table 5.8: Confusion matrix between reference map and filtered classification map from unmixing results.

\begin{tabular}{|c|c|c|c|c|c|c|c|c|c|}
\hline & \multicolumn{6}{|c|}{ Reference Data } & \multirow[b]{2}{*}{ Totals } & \multirow[b]{2}{*}{$\begin{array}{c}\text { User's } \\
\text { Accuracy (\%) }\end{array}$} \\
\hline & & $\begin{array}{c}\text { Asphalt and } \\
\text { Bitumen }\end{array}$ & $\begin{array}{c}\text { Meadows } \\
\text { and Soil }\end{array}$ & $\begin{array}{c}\text { Gravel } \\
\text { and Brick }\end{array}$ & Trees & Metal Sheet & Shadow & & \\
\hline \multirow{8}{*}{$\begin{array}{l}\text { Class } \\
\text { Map }\end{array}$} & $\begin{array}{l}\text { Asphalt and } \\
\text { Bitumen }\end{array}$ & 4168 & 327 & 768 & 42 & 20 & 208 & 5533 & 75.33 \\
\hline & $\begin{array}{l}\text { Meadows } \\
\text { and Soil }\end{array}$ & 0 & 21355 & 134 & 1106 & 6 & 10 & 22611 & 94.45 \\
\hline & $\begin{array}{c}\text { Gravel } \\
\text { and Brick }\end{array}$ & 0 & 493 & 4538 & 10 & 10 & 1 & 5052 & 89.83 \\
\hline & Trees & 0 & 208 & 0 & 1751 & 0 & 5 & 1964 & 89.15 \\
\hline & Metal Sheet & 0 & 0 & 0 & 0 & 1259 & 4 & 1263 & 99.68 \\
\hline & Shadow & 0 & 0 & 0 & 0 & 0 & 383 & 383 & 100 \\
\hline & Totals & 4168 & 22383 & 5440 & 2909 & 1295 & 611 & 36806 & \\
\hline & $\begin{array}{c}\text { Producer's } \\
\text { Accuracy (\%) }\end{array}$ & 100 & 95.41 & 83.41 & 60.19 & 97.22 & 62.68 & & $\begin{array}{c}\text { Overall } \\
\text { Accuracy }=90.89\end{array}$ \\
\hline
\end{tabular}

Second, we compare with global unmixing over the low dimensional image without segmentation.

Finally, we compare with results from the quadtree approach without superpixel segmentation as in [5]. Comparison will be from the results of Urban scene and it will be based on the confusion matrix and the kappa coefficient.

\subsubsection{Comparing With the Global Approach}

In this approach, estimation of endmembers will be applied to the full hyperspectral image. We will use the Gram method to estimate the number of endmembers. Endmember extraction will then be applied to the full scene and later abundances will be estimated using the NNSLO algorithm 
mentioned before.

The Gram method estimated 16 endmembers in the scene. Abundance estimation is performed using the NNSLO. We then grouped abundances into five information classes that describe the scene. The results are shown in Figures 5.27 and 5.28.

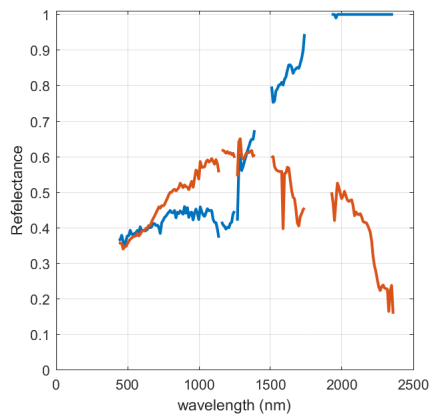

Road

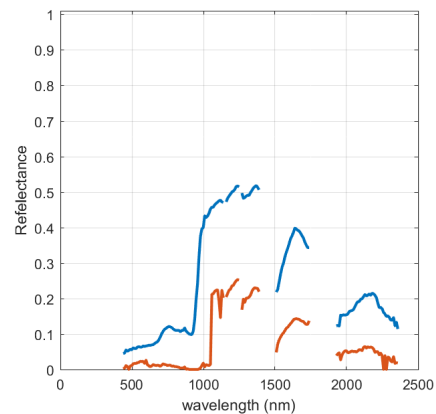

Grass

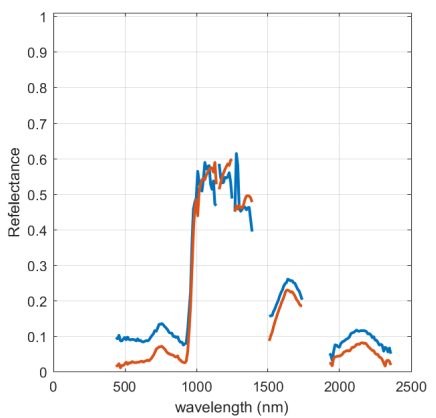

Trees

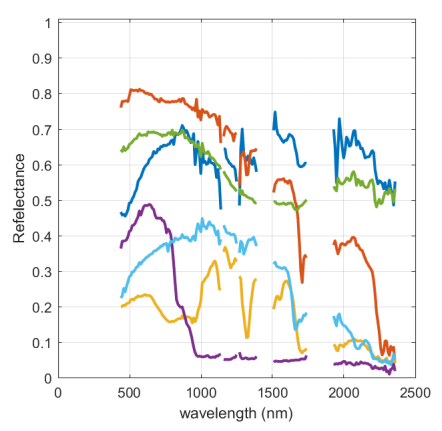

Roof

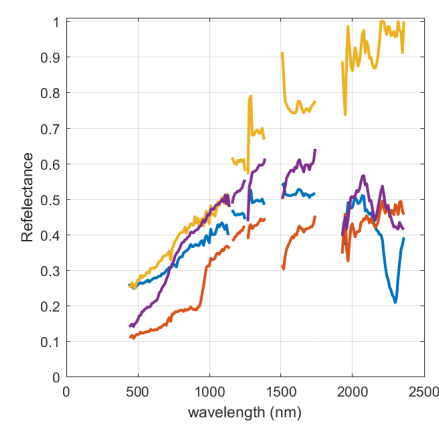

Dirt

Figure 5.27: Spectral endmembers extracted from the Urban scene using global analysis

By visually comparing the spectral signatures extracted using the global method to the ones available from the reference data (Figure 5.12), the extracted endmembers do not look similar to the reference data in terms of shape. This is easily noticed for the Road class.

A class map was generated based on the abundance map using winner takes it all criteria. The resulting class map is shown in Figure 5.29. Pixels with black label are the ones where all abundances are of a small value and none is higher than $50 \%$. The class map generated from global analysis poorly captures the road class and it labeled it as Dirt instead.

The class map is compared to the reference data class map using the confusion matrix as 


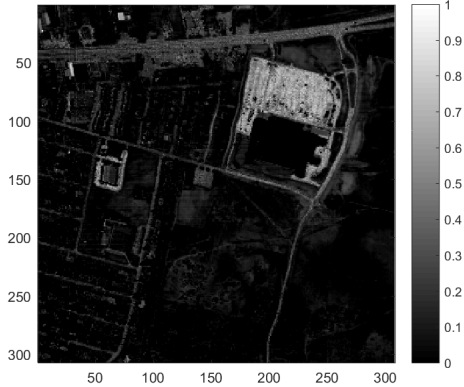

Road

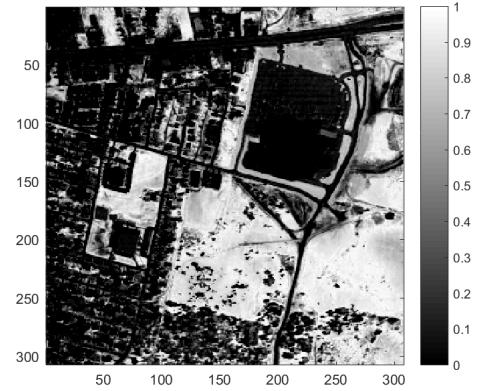

Grass

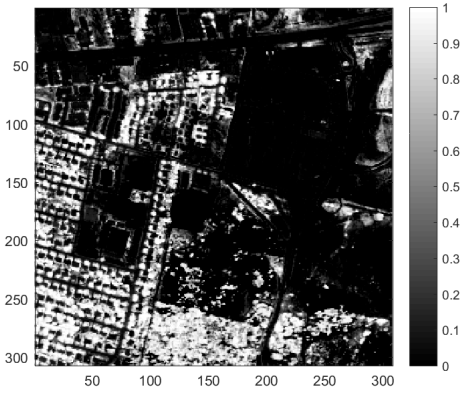

Trees

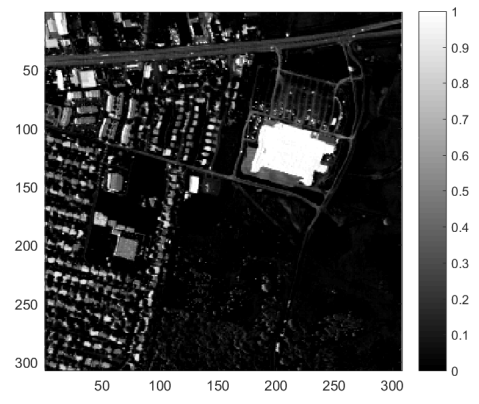

Roof

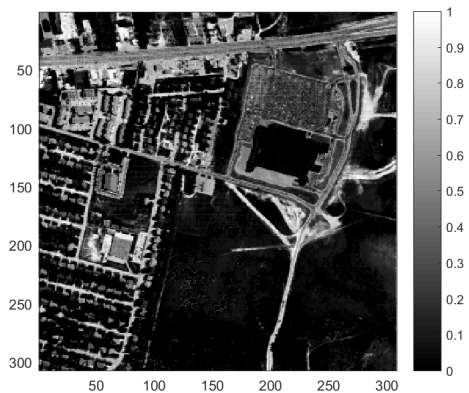

Dirt

Figure 5.28: Abundance maps extracted from the Urban scene using global analysis

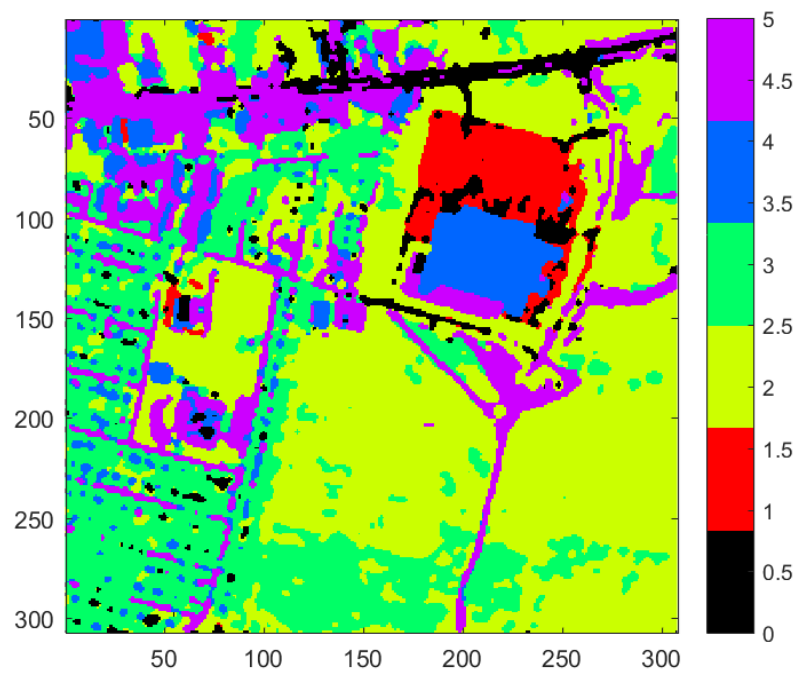

Figure 5.29: Class map generated using Global approach for Urban scene.

shown in Table 5.9. The producer's accuracy's are quite low compared to the ones in Table 5.5 specially the Road class, which is expected considering the class map generated from the global analysis. Also, the overall accuracy has dropped to $78 \%$ compared to $88.97 \%$ using our approach. 
We also computed the kappa coefficient for both methods. The global approach Kappa resulted in $69.2 \%$ which is considered relatively low compared to our approach that scores $85.01 \%$.

Table 5.9: Confusion matrix between reference map and generated classification map from global approach.

\begin{tabular}{|c|c|c|c|c|c|c|c|c|}
\hline & \multicolumn{5}{|c|}{ Reference Data } & \multirow[b]{2}{*}{ Totals } & \\
\hline & & Road & Grass & Trees & Roof & Dirt & & $\begin{array}{c}\text { User's } \\
\text { Accuracy }(\%)\end{array}$ \\
\hline \multirow{7}{*}{$\begin{array}{l}\text { Class } \\
\text { Map }\end{array}$} & Road & 3717 & 18 & 8 & 21 & 1 & 3765 & 98.73 \\
\hline & Grass & 1029 & 34841 & 3275 & 177 & 1961 & 41283 & 84.40 \\
\hline & Trees & 393 & 304 & 21325 & 1017 & 159 & 23198 & 91.93 \\
\hline & Roof & 1121 & 144 & 359 & 5127 & 110 & 6861 & 74.73 \\
\hline & Dirt & 7480 & 918 & 823 & 395 & 4886 & 14502 & 33.69 \\
\hline & Totals & 13740 & 36225 & 25790 & 6737 & 7117 & 89609 & \\
\hline & $\begin{array}{c}\text { Producer's } \\
\text { Accuracy }(\%)\end{array}$ & 27.05 & 96.18 & 82.69 & 76.10 & 68.65 & & $\begin{array}{c}\text { Overall } \\
\text { Accuracy }=78.00\end{array}$ \\
\hline
\end{tabular}

\subsubsection{Comparing with the Global Low Dimensional Approach with no Segmentation}

In this approach, endmember extraction will be performed on the LD image without quadtree segmentation. The Gram method is used to estimate the number of endmembers. Endmembers will be extracted from the LD image and later abundances will be estimated using the NNSLO.

The Gram method estimated 10 endmembers in the LD scene. Abundance estimation is performed using NNSLO. We then formed the 5 information classes that describe the scene. The results are shown in Figures 5.30 and 5.31.

By visually comparing the spectral signatures extracted using the global method on the LD image to the ones available from the reference data (Figure 5.12), the extracted endmembers look similar to the reference data in terms of shape (except for the Road and Roof classes).

A class map was generated based on the abundance map using winner takes it all criteria. The resulting class map is shown in Figure 5.32. Pixels with black label are the ones where all abundances are of a small value and none is higher than 50\%. The class map generated from 


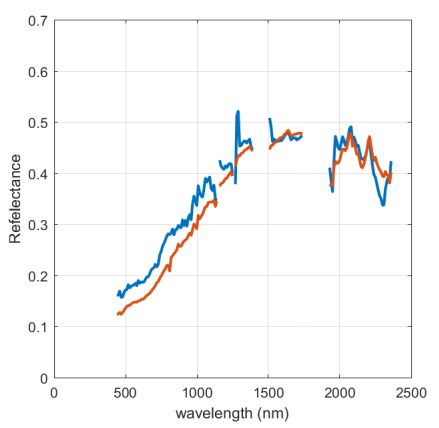

Road

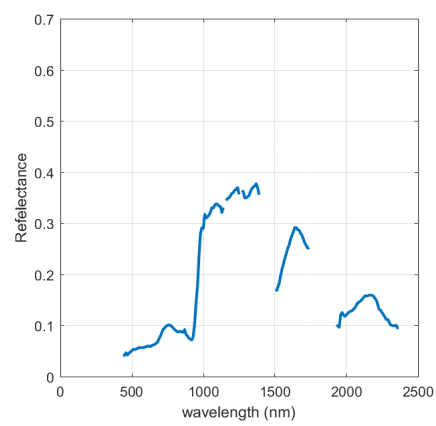

Grass

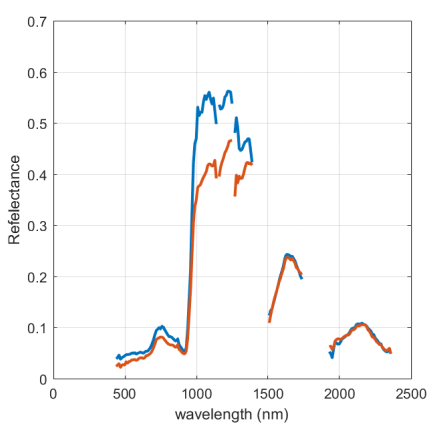

Trees

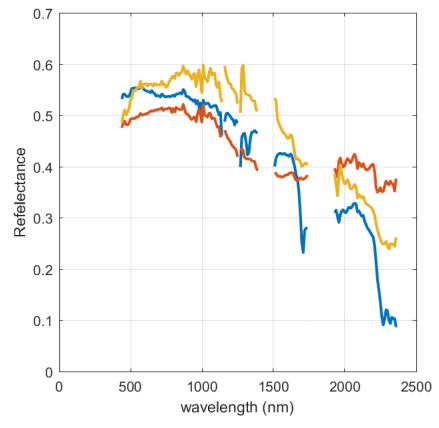

Roof

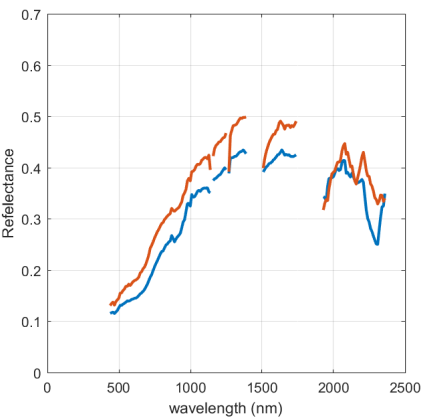

Dirt

Figure 5.30: Spectral endmembers extracted from the urban scene using global analysis on the LD image

global analysis poorly captures the Dirt class and it labeled it as Road instead.

The class map is compared to the reference data class map using the confusion matrix as shown in Table 5.10. The producer's accuracies are quite low compared to the ones in Table 5.5 specially the Dirt class, which is expected considering the class map generated from the global analysis. Also, the overall accuracy has dropped to $79.2 \%$ compared to $88.97 \%$ using our approach.

We also computed the kappa coefficient for both methods. The LD global approach Kappa resulted in $71.57 \%$ which is considered relatively low compared to our approach that scores $85.01 \%$.

\subsubsection{Comparing With the Quadtree Approach and no Superpixel Segmentation}

Here, we compare our approach with the one suggested by [5], the full image is divided into spectrally homogeneous tiles using the Quadtree method. The segmentation criteria is based on the 


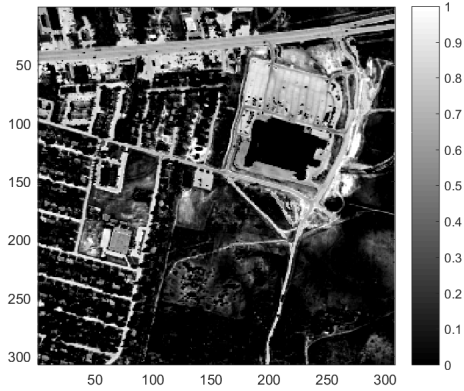

Road

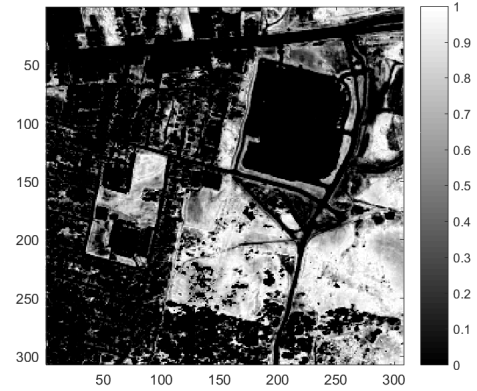

Grass

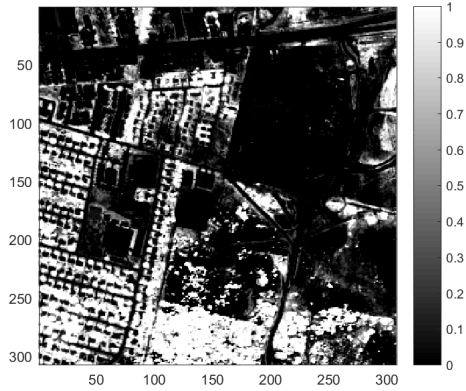

Trees

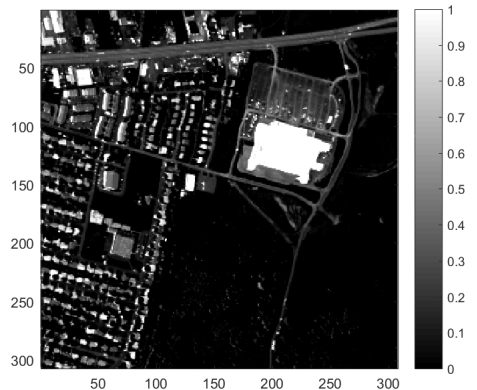

Roof

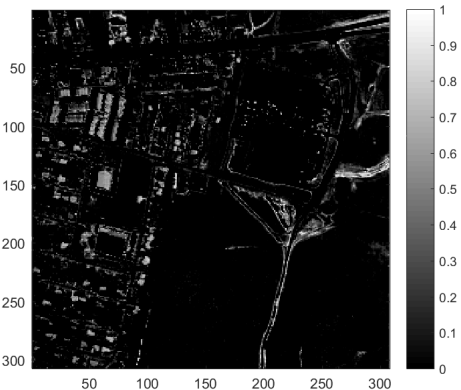

Dirt

Figure 5.31: Abundance maps extracted from the urban scene using the global analysis on the LD image.

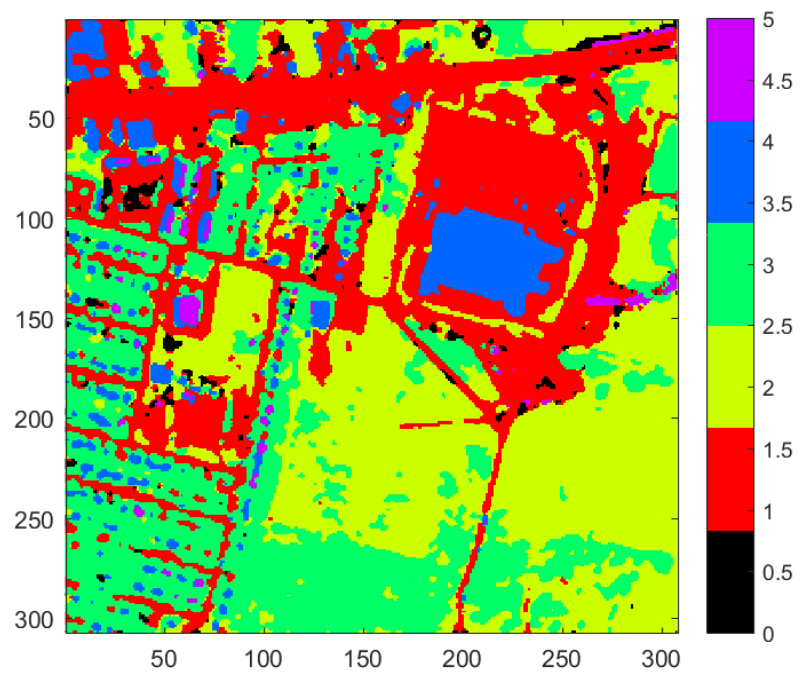

Figure 5.32: Class map generated using Global analysis on the LD approach

Shannon entropy (Eq. 2.16). If the entropy for each tile is higher than $90 \%$ of the full image entropy, the tile will be divided into four quadrants, or else (lower than 90\%) it will become a tree leaf. Figure 5.33(a) Shows the final result of the segmentation. 
Table 5.10: Confusion matrix between reference map and generated classification map from the global LD approach.

\begin{tabular}{|c|c|c|c|c|c|c|c|c|}
\hline & \multicolumn{5}{|c|}{ Reference Data } & \multirow[b]{2}{*}{ Totals } & \multirow[b]{2}{*}{$\begin{array}{c}\text { User's } \\
\text { Accuracy }(\%)\end{array}$} \\
\hline & & Road & Grass & Trees & Roof & Dirt & & \\
\hline \multirow{5}{*}{$\begin{array}{l}\text { Class } \\
\text { Map }\end{array}$} & Road & 16476 & 4280 & 2256 & 756 & 5993 & 29761 & 55.36 \\
\hline & Grass & 70 & 28905 & 592 & 132 & 84 & 29783 & 97.05 \\
\hline & Trees & 117 & 2399 & 22656 & 841 & 185 & 26198 & 86.48 \\
\hline & Roof & 738 & 76 & 315 & 4748 & 66 & 5943 & 79.89 \\
\hline & Dirt & 0 & 53 & 25 & 289 & 594 & 961 & 61.81 \\
\hline & Totals & 17401 & 35713 & 25844 & 6766 & 6922 & 92646 & \\
\hline & $\begin{array}{c}\text { Producer's } \\
\text { Accuracy (\%) }\end{array}$ & 94.68 & 80.94 & 87.66 & 70.17 & 8.58 & & $\begin{array}{c}\text { Overall } \\
\text { Accuracy }=79.2\end{array}$ \\
\hline
\end{tabular}

The number of endmembers per tile is extracted using the Gram method. The results are shown in Figure 5.33(b). A total number of 114 endmembers were extracted using SVDSS. Abundance estimation using the NNSLO is applied to the full image using the extracted endmembers. Clustering using the angle distance of 0.005 led to 30 endmember classes. Information classes were assigned using the reference data as before. Results are shown in Figures 5.34 and 5.35.

The class map was generated same approach based on majority voting and $50 \%$ threshold. Figure 5.36 shows the resulting class map from the extracted endmembers. The map is consider-

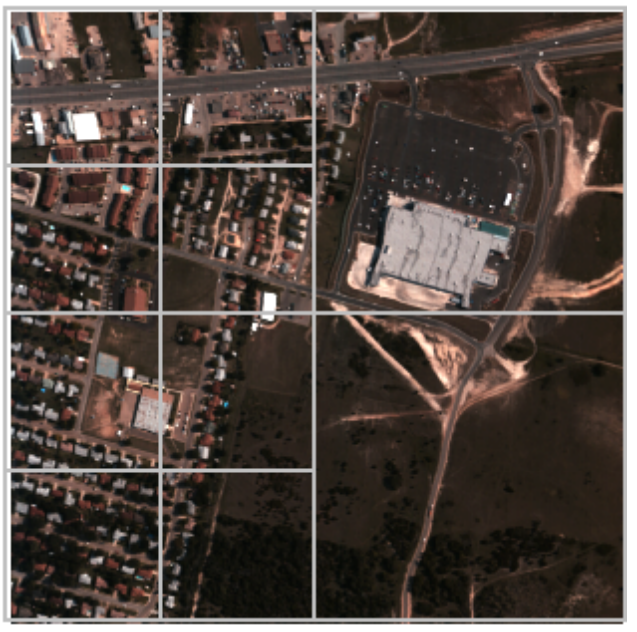

(a)

\begin{tabular}{|c|c|c|}
\hline 14 & 12 & \multirow{2}{*|}{14} \\
\cline { 2 - 2 } 11 & 11 & \\
\hline 11 & 11 & 10 \\
\hline 10 & 10 & 10 \\
\hline
\end{tabular}

(b)

Figure 5.33: (a) Quadtree Image Segmentation of Urban scene; (b) Number of endmembers per tile (total $=114)$. 


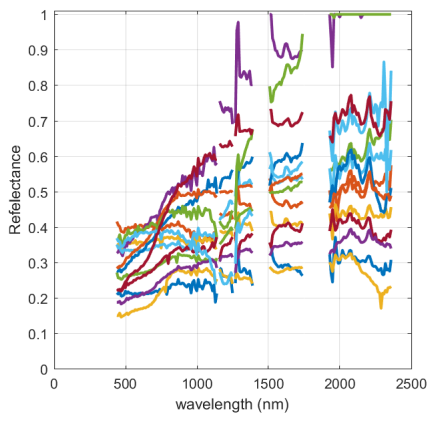

Road

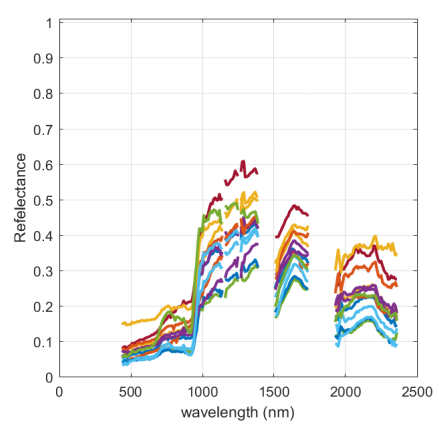

Grass

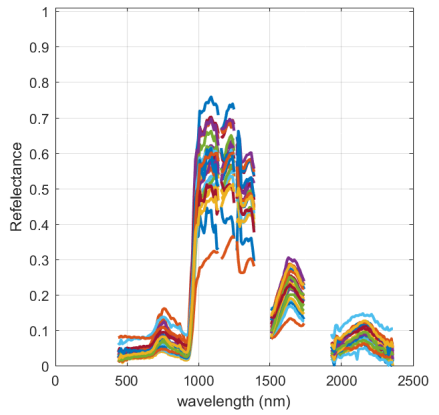

Trees

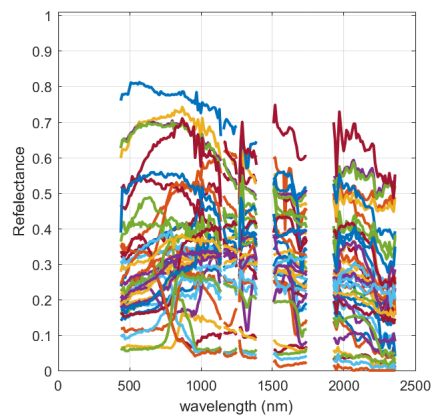

Roof

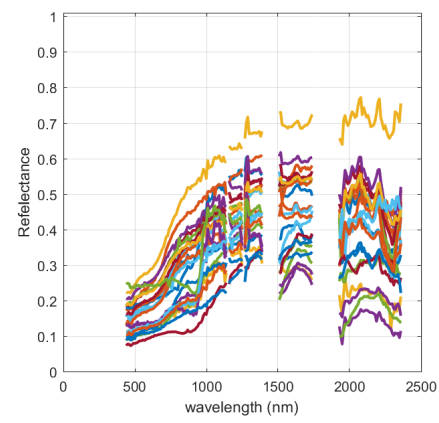

Dirt

Figure 5.34: Spectral information classes extracted from the urban scene using the Quad-tree Approach

ably better than the one with the global approach and looks very similar with one obtained with our approach. To better compare the results of the full pixel quadtree with the ones obtained from the SLIC approach, the confusion matrix is constructed as shown in Table 5.11.

Table 5.11: Confusion matrix between reference map and generated classification map from the quadtree approach.

\begin{tabular}{|c|c|c|c|c|c|c|c|c|}
\hline & \multicolumn{5}{|c|}{ Reference Data } & \multirow[b]{2}{*}{ Totals } & \\
\hline & & Road & Grass & Trees & Roof & Dirt & & $\begin{array}{c}\text { User's } \\
\text { Accuracy (\%) }\end{array}$ \\
\hline \multirow{7}{*}{$\begin{array}{l}\text { Class } \\
\text { Map }\end{array}$} & Road & 16080 & 1973 & 1215 & 282 & 444 & 19994 & 80.42 \\
\hline & Grass & 51 & 31226 & 1055 & 78 & 320 & 32730 & 95.40 \\
\hline & Trees & 136 & 1190 & 22117 & 460 & 221 & 24124 & 91.68 \\
\hline & Roof & 793 & 125 & 888 & 6008 & 1157 & 8971 & 66.97 \\
\hline & Dirt & 54 & 963 & 35 & 9 & 4752 & 5813 & 81.75 \\
\hline & Totals & 17114 & 35477 & 25310 & 6837 & 6894 & 91632 & \\
\hline & $\begin{array}{c}\text { Producer's } \\
\text { Accuracy }(\%)\end{array}$ & 93.96 & 88.02 & 87.38 & 87.87 & 68.93 & & $\begin{array}{c}\text { Overall } \\
\text { Accuracy }=87.51\end{array}$ \\
\hline
\end{tabular}




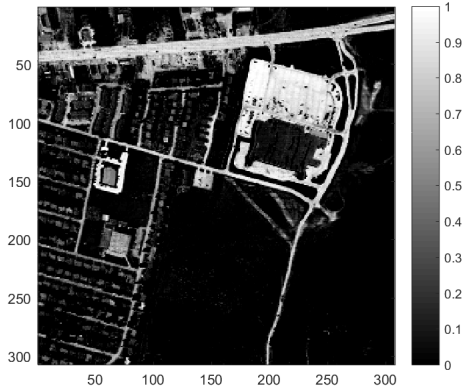

Road

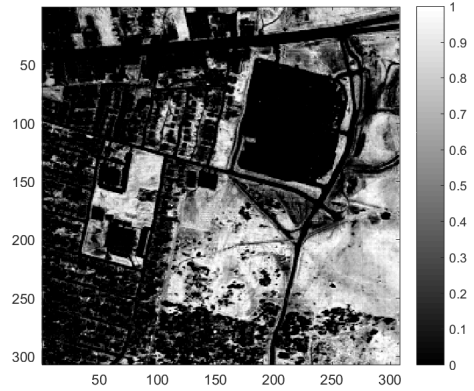

Grass

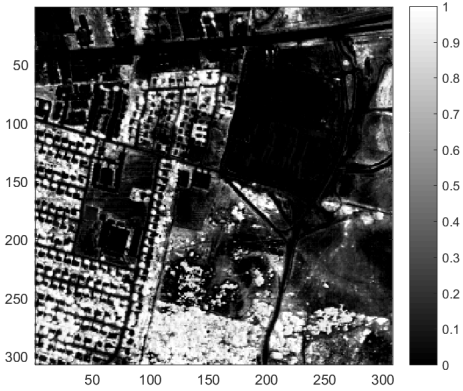

Trees

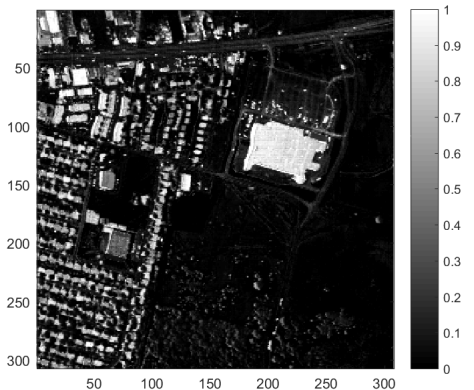

Roof

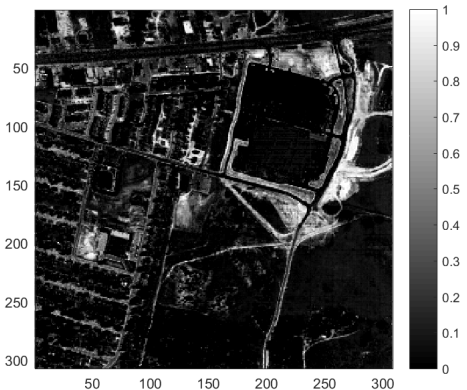

Dirt

Figure 5.35: Abundance maps extracted from the urban scene using the Quadtree approach.

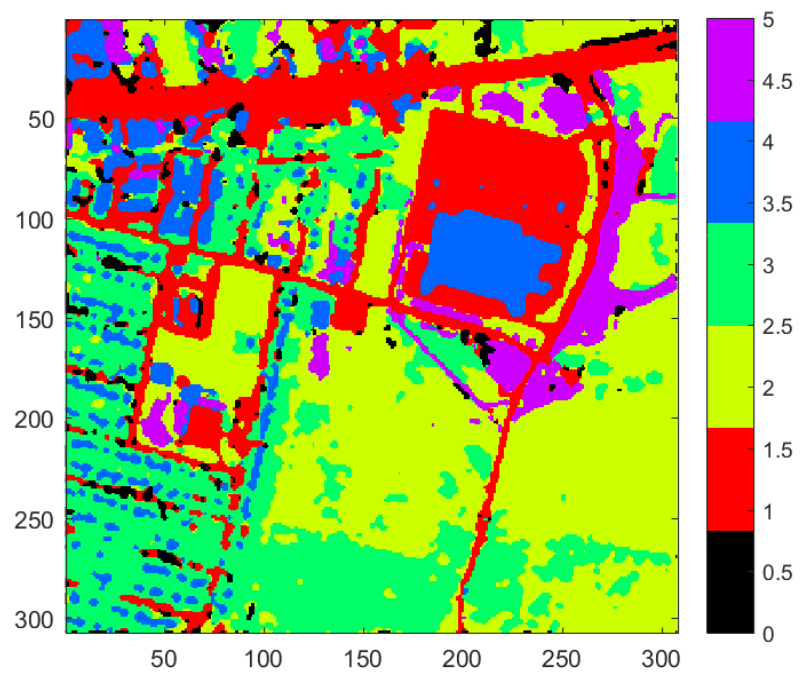

Figure 5.36: Class map generated using the Quadtree approach

Table 5.11 shows an agreement of $87.51 \%$ between the generated class map using quadtree approach and the reference data class map, this is considered as a good improvement to the one obtained using global analysis (78\%), The producer's accuracy of the Road class has improved 
significantly to $93.96 \%$ when compared to the $27.05 \%$ of the global method. However, the producer's accuracy of the Grass class dropped to $88.02 \%$. This not considered a significant change.

We compared Tables 5.11 and 5.5 to assess the performance of our approach. The overall accuracy for both methods is very close. Still our method scores a higher accuracy with $88.97 \%$. The producer's accuracy for the majority of classes is higher in our approach. Except for the Road class, the producer's accuracy was higher in the quadtree approach with a score of $93.96 \%$.

The results above show that our approach performs well compared to the approach of [5]. To finalize the comparison, the Kappa coefficient was calculated for the quadtree approach and the value was $83.03 \%$, which is smaller than the one obtained using our approach $(85.01 \%)$.

An important point to compare is the computational issue. However, we didn't perform a full computational analysis but we just compared the simulation time that was taken for the segmentation and endmember extraction steps. The approach of [5] took 240 seconds to produce the segmentation and sometimes the segmentation crashes due to memory issues in large scenes. While our approach only took 37 seconds to segment and extract the endmembers. The computer used to perform ths simulation has an 8-core AMD Athlon processor with 32GB of RAM and simulations were performed using Matlab R2017a.

\subsubsection{Additional Comparison}

Tables 5.12 and 5.13 show a summary of results obtained using the four approaches. Table 5.12 shows the producer's and user's accuracies of each class for the four methods, while Table 5.13 shows a comparison based on Kohen's Kappa and the Overall accuracies for the four methods.

Table 5.12: Producer's and User's Accuracies for the 4 methods on the Urban data set

\begin{tabular}{|c|c|c|c|c|c|c|c|c|}
\cline { 2 - 9 } \multicolumn{1}{c|}{} & \multicolumn{2}{c|}{ LD + QT } & \multicolumn{2}{c|}{ Full + QT } & \multicolumn{2}{c|}{ LD Global } & \multicolumn{2}{c|}{ Full Global } \\
\cline { 2 - 9 } \multicolumn{1}{c|}{} & P.A\% & U.A\% & P.A\% & U.A\% & P.A\% & U.A\% & P.A\% & U.A\% \\
\hline Road & 88.93 & 86.6 & 93.96 & 80.42 & 94.68 & 55.36 & 27.05 & 98.73 \\
\hline Grass & 89.58 & 97.36 & 88.02 & 95.40 & 80.94 & 97.05 & 96.18 & 84.40 \\
\hline Trees & 90.86 & 89.03 & 87.38 & 91.68 & 87.66 & 86.48 & 82.69 & 91.93 \\
\hline Roof & 92.22 & 64.73 & 87.87 & 66.97 & 70.17 & 79.89 & 76.10 & 74.73 \\
\hline Dirt & 76.08 & 88.90 & 68.93 & 81.75 & 8.58 & 61.81 & 68.65 & 33.69 \\
\hline
\end{tabular}


Table 5.13: Kohen's Kappa and overall accuracies for the 4 methods

\begin{tabular}{|c|c|c|}
\cline { 2 - 3 } \multicolumn{1}{c|}{} & Kohen's Kappa (\%) & Overall Accuracy (\%) \\
\hline LD + QT & 85.01 & 88.97 \\
\hline Full + QT & 83.03 & 87.51 \\
\hline LD Global & 71.57 & 79.20 \\
\hline Full Global & 69.02 & 78.00 \\
\hline
\end{tabular}

All classes were detected using the four different approaches as seen in Table 5.12. The Road class was poorly captured using the full global approach with a producer's accuracy of $27.05 \%$, this also can be seen from the class map in Figure 5.29 where most of the Road regions were labeled as Dirt. The other three methods successfully captured the Road Class with a very high Producer's accuracy (more than $88 \%$ ).

The Grass class was captured in all cases with a very high accuracy, this due to the fact that this class occupies a very large spatial area that it will be easily captured even with global approaches, same comment can also be made regarding the Roof class.

The Dirt class was poorly captured using the LD global approach, by referring to Figure 5.30, the Road class endmembers, in fact, belongs to the Dirt class, however, they have more contribution to the Road class as shown in Figure 5.31 and that is why they were not labeled as Dirt but instead they were labeled as Road.

Table 5.12 only shows class by class comparison. The results of Table 5.13 give an overall performance of each method. The evaluation is mainly based on the Kappa statistic since the overall accuracy is only an average that does not reveal if error was evenly distributed between classes or if some classes were really bad and some really good. Our method scored the highest Kappa with $85.01 \%$ which is a little higher than the Kappa obtained by the full quadtree method $(83.03 \%)$. Adding to this comparison, the time needed to extract the endmembers was considerably lower (almost 20\%) than the one needed by the full quadtree. 


\subsection{Summary}

Hyperspectral images from different sensors were used to study the performance of the proposed unmixing approach based on the SLIC segmentation with regional analysis. HYDICE Urban and ROSIS Pavia University are presented. We used both visual (qualitative) and quantitative analysis (based on the confusion matrix) to evaluate the performance of our algorithm. The quantitative approach was based on the confusion matrix between the reference data class map and the generated class map from our approach. In both data sets, the value of Kappa was higher than $80 \%$, which is considered as an almost perfect agreement between our class map and the reference data.

We then compare our results over the Urban scene with previous methods used in hyperspectral unmixing, the reason of selecting Urban scene is due to the rich available reference data and the fact that the scene occupies the full image dimensions, unlike the Pavia University scene that has a lot of background that is discarded. The comparison was based on the results of the confusion matrix for three unmixing scenarios compared to the one obtained from our method. Our approach showed the best results in terms of overall accuracy and the Kappa statistic. 


\section{Chapter 6}

\section{EXPERIMENTS WITH FORT A.P. HILL DATA SET}

\subsection{Introduction}

This chapter is dedicated to experiments conducted using the data collected with the Airborne Vis-

ible and Infrared Imaging Spectrometer (AVIRIS) over Fort A. P. Hill, Virginia USA in September of 2001. The image is heavily forested with a mix of deciduous and coniferous species native to the mid-Atlantic region. It consists of 224 bands from 380 to $2500 \mathrm{~nm}$ with a spatial resolution of 3.5 meters. The image has $512 \times 540$ pixels. Only 155 bands will be considered in the unmixing analysis. Water absorption and noisy bands were removed (bands 1-5, 31-33, 77, 96, 102-117, 150-178, 211-224) [8]. Figure 6.1(a) shows the RGB composition using bands 37, 20 and 17. A refernece classification map from [8] is used here and shown in Figure 6.1(b). The reference classification map shows 14 different classes: three types of soil (soil ag field \#1, soil ag field \#2, soil ag field \#3), eight types of vegetation (summer deciduous forest, loblolly pine, autumn deciduous \#1, autumn deciduous \#2, autumn deciduous \#3, green ag field \#1, shaded vegetation, and grass field), two man made classes (generic road and gravel), and river water. In addition, there is a reference spectral library documented in [8] and is shown in Figure 6.2. Only the plots for the spectral library are available, so they are visually compared with the spectral endmember classes obtained with the proposed approach. The spectral library of [8] contains image-derived endmembers.

\subsection{Justification}

The Fort A.P. Hill data set is considered hard for hyperspectral unmixing due to many reasons. First, the endmembers are very similar, specially when considering the vegetation classes, as an overall there are eight types which many have very similar spectral features. Second, the reference class map of the scene is not very clear, and has many pixels of certain classes (summer deciduous forest for example) are scattered in the image and do not have any spatial correlation among the 


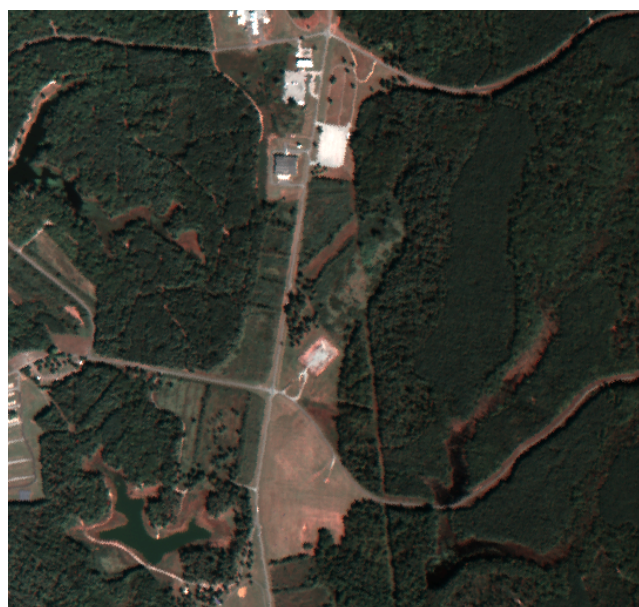

(a)

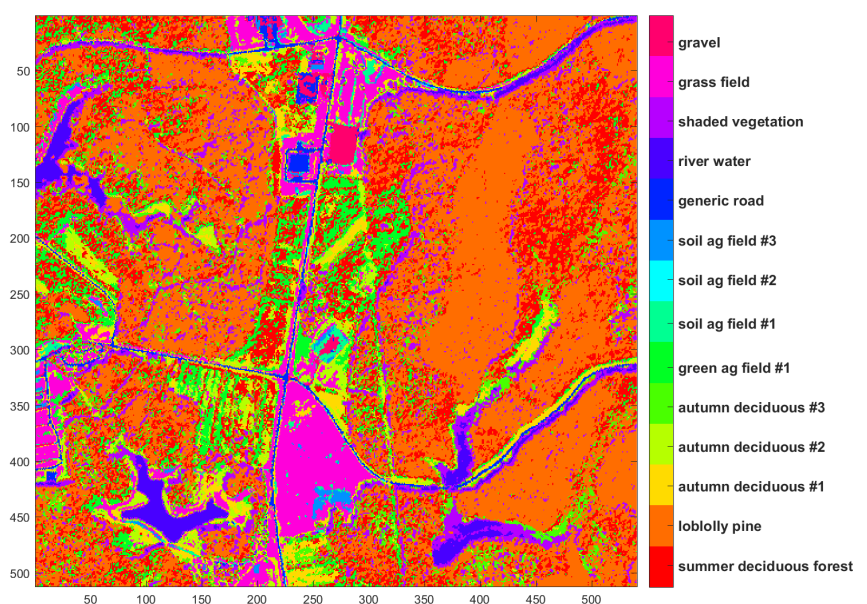

(b)

Figure 6.1: A.P. Hill Hyperspectral image: (a) RGB composition; (b) Reference data [8].

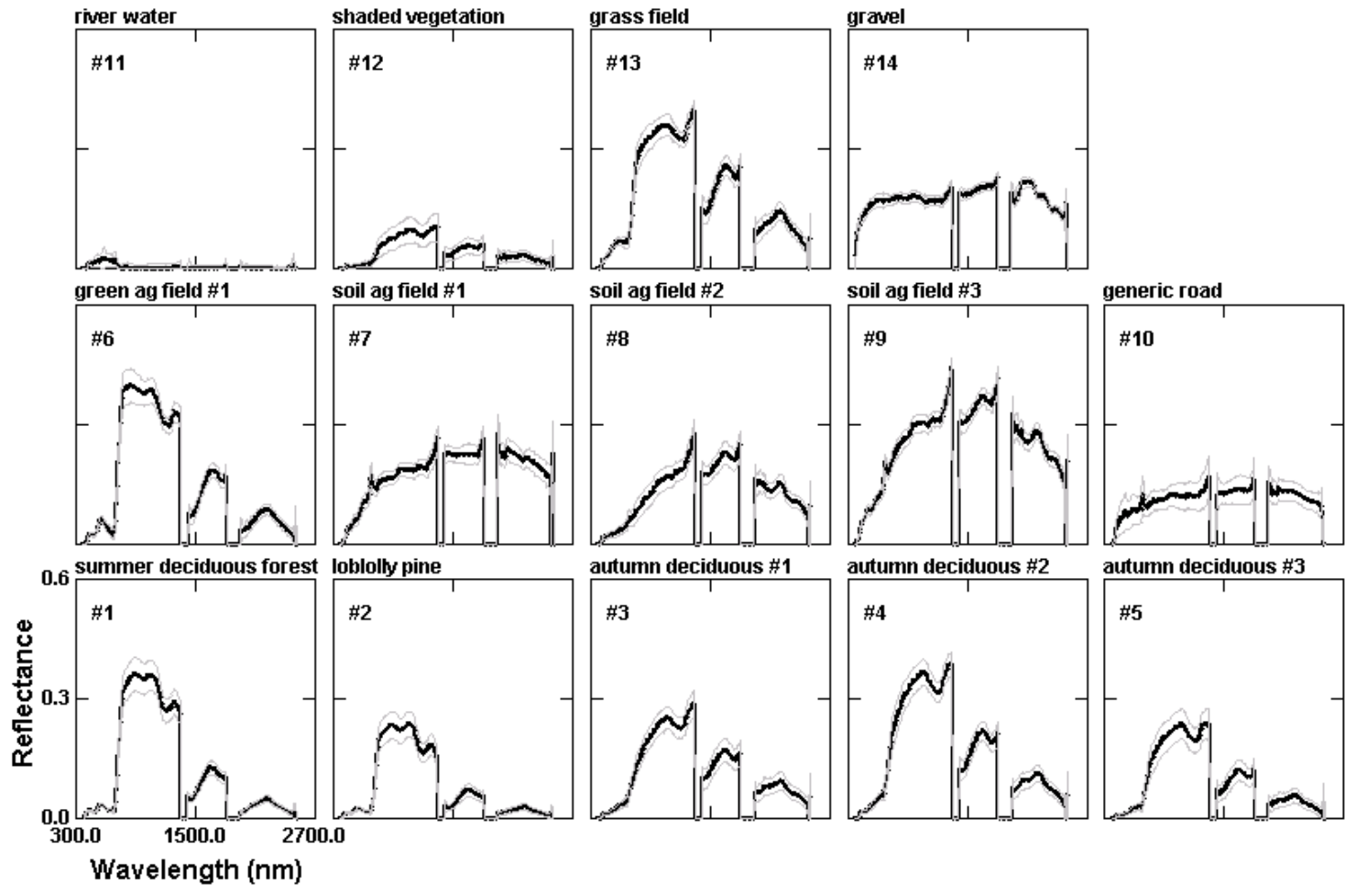

Figure 6.2: Spectral library for A.P. Hill. Figure from [8]

class pixels. Usually, scattered pixels are unwanted when forming class maps (see for instance the Pavia University Reference data in Figure 5.2(b)). Finally, the lack of availability of valid spectral library of the scene, only plots are available and one cannot perform quantitative analysis (angle distance, for example) between extracted endmembers and reference spectral library. 


\subsection{Creating New Reference Map}

In order to overcome the difficulties mentioned in the previous section, we created a new class map that describes the scene in more descriptive manner. We first merged spectral classes that describe the same land cover. For example, we combined soil ag field \#1, soil ag field \#2, soil ag field \#3 together to form a new class named Soil ag Field. This step will reduce the number of classes from 14 classes into 10 classes. Second, to eliminate scattered pixels, we applied to the new class map a $3 \times 3$ median filter. This particular size is relatively small and won't affect the spatial information in the scene. Figure 6.3 shows the result of the two steps mentioned before. Clearly the class map now looks more clean and informative and one can distinguish between classes easily by just visual inspection when compared to Figure 6.1(b).

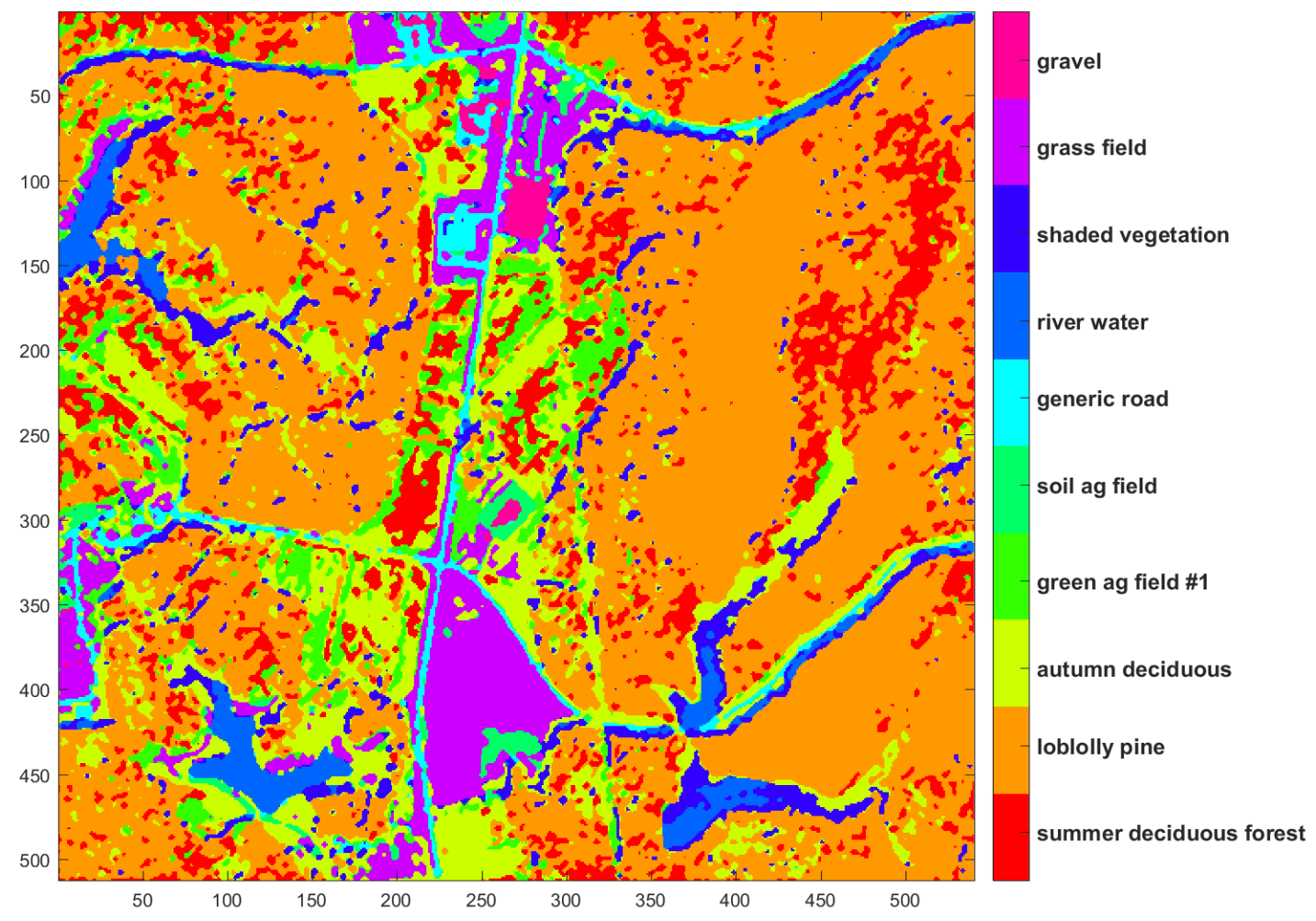

Figure 6.3: New Reference class map after applying the steps discussed in Section 6.3

The new reference map shown in Figure 6.3 now contains six classes of vegetation (summer deciduous forest, loblolly pine, autumn deciduous, green ag field \#1, shaded vegetation, and grass field) and one Soil class instead of three. The merging of spectral classes reduced the complexity 
of the image. However, few classes have a large quantity of pixels and spatial occupancy (mostly the Loblolly Pine class). Some classes like the generic road and shaded vegetation have a very low spatial occupancy and pixel count. But still, having a unique signature for the gravel and the generic road classes will help identifying the materials. However, the shaded vegetation class has a very low pixel count (or percentage) and the spectral signature looks similar to other vegetation classes. This may affect our analysis after the unmixing. It is suggested, later on, to merge some vegetation classes to improve our analysis. Table 6.1 shows the number of pixels per class and their percentage in the image.

Table 6.1: A.P. Hill class pixel counts and percentages

\begin{tabular}{|c|c|c|}
\hline Class & Pixels count & Percentage \% \\
\hline summer deciduous forest & 30511 & 11.04 \\
\hline loblolly pine & 141679 & 51.24 \\
\hline autumn deciduous & 39939 & 14.45 \\
\hline green ag field \#1 & 13861 & 5.01 \\
\hline soil ag field & 3808 & 1.38 \\
\hline generic road & 5917 & 2.14 \\
\hline river water & 7257 & 2.62 \\
\hline shaded vegetation & 13845 & 5.01 \\
\hline grass field & 18035 & 6.52 \\
\hline gravel & 1628 & 0.59 \\
\hline
\end{tabular}

\subsection{Unmixing Analysis}

A.P. Hill is analyzed using the proposed approach. First, The SLIC algorithm (Section 4.2) is applied to the full image for dimensionality reduction. Each superpixel will be represented by its mean spectra to form a low dimensional (LD) representation of the image. The size of the LD image is $32 \times 34 \times 155$. Figure 6.4 (a) shows the result of the SLIC segmentation.

The LD image of spectral means in Figure 6.4(b) is partitioned using the quadtree method based on the Shannon entropy as shown in Figure 6.5. Endmember extraction using SVDSS will be performed on each tile. The number of endmembers will be estimated using the Gram method. 


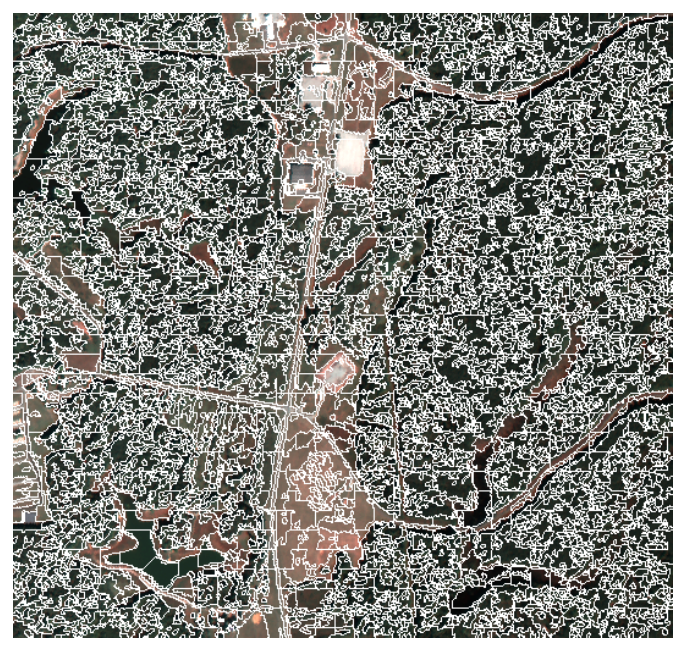

(a)

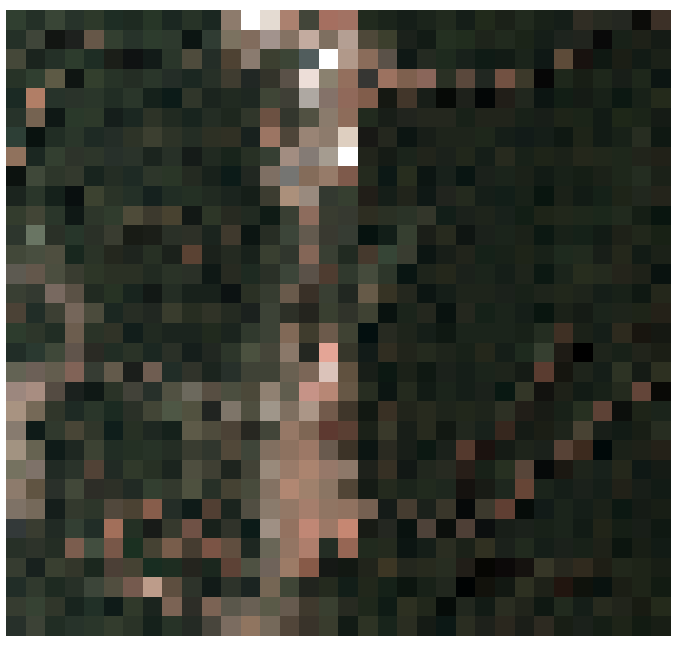

(b)

Figure 6.4: (a) SLIC segmentation of A.P. Hill image (region size = 16); (b) RGB display of the low dimensional superpixel image.

A total of 58 endmembers were extracted. Clustering analysis using the angle distance of 0.001 resulted in having 27 endmember classes. We used 0.001 here since most of the image endmembers are vegetation of different types and we are trying to separate them to reduce confusion between vegetation endmember classes.

\begin{tabular}{|c|c|c|c|}
\hline $\begin{array}{l}\mathrm{L} 1.1 \\
8 \times 8 \\
4 \mathrm{Ems}\end{array}$ & $\begin{array}{c}\text { L } 1.2 \\
8 \times 8 \\
5 \text { Ems }\end{array}$ & $\begin{array}{c}\text { L } 2.1 \\
8 \times 8 \\
4 \text { EMs }\end{array}$ & $\begin{array}{c}\mathrm{L} 2.2 \\
\mathbf{8} \times \mathbf{8} \\
3 \mathrm{Ems}\end{array}$ \\
\hline $\begin{array}{c}\text { L } 1.3 \\
8 \times 8 \\
4 \text { EMs }\end{array}$ & $\begin{array}{c}\text { L } 1.4 \\
8 \times 8 \\
4 \mathrm{EMs}\end{array}$ & $\begin{array}{c}\text { L } 2.3 \\
8 \times 8 \\
3 \text { EMs }\end{array}$ & $\begin{array}{c}\text { L } 2.4 \\
8 \times 8 \\
3 \mathrm{Ems}\end{array}$ \\
\hline $\begin{array}{l}\text { L } 3.1 \\
8 \times 8 \\
4 \text { Ems }\end{array}$ & $\begin{array}{c}\text { L } 3.2 \\
8 \times 8 \\
3 \mathrm{EMs}\end{array}$ & $\begin{array}{c}\text { L } 4.1 \\
8 \times 8 \\
4 \text { EMs }\end{array}$ & $\begin{array}{c}\text { L } 4.2 \\
8 \times 8 \\
3 \mathrm{Ems}\end{array}$ \\
\hline $\begin{array}{c}\text { L } 3.3 \\
8 \times 8 \\
4 \text { EMs }\end{array}$ & $\begin{array}{c}\text { L } 3.4 \\
8 \times 8 \\
3 \mathrm{EMs} \\
\end{array}$ & $\begin{array}{l}\text { L } 4.3 \\
8 \times 8 \\
4 \text { Ems }\end{array}$ & $\begin{array}{r}\text { L } 4.4 \\
8 \times 8 \\
3 \text { EMs }\end{array}$ \\
\hline
\end{tabular}

Figure 6.5: Partitioning using quadtree $(\mathrm{EMs}=$ Endmembers $)$.

In the end, we formed information classes based on the land covers that describe the scene. The spectral class labeling was based on the visual analysis of the spectral endmembers shape and their abundance distribution. The information class maps are shown in Figure 6.6, while Figure 
6.7 shows the signatures for each class.

In order to form a classification map from the information abundance maps obtained in Figure 6.6, we follow the same procedure used in Section 5.4.1.2. The resulting class map (Figure 6.8) will be compared to the reference map of Figure 6.3 using the confusion matrix shown in Table 6.2 .

The classification map obtained using the proposed approach has an overall accuracy of 67.64\% which is low when compared to the data sets analyzed in Chapter 5. As expected, vegetation classes with low pixels coverage (shaded vegetation for example) have a very low producer's accuracy. The gravel, on the other hand, has a very good producer's accuracy even though the number of pixels in the image are very low (the percentage is less than 1 for this particular class). That is due to the fact that the spectral signature of this class is unique.

Table 6.2: Confusion matrix between reference map and generated classification map from unmixing results.

\begin{tabular}{|c|c|c|c|c|c|c|c|c|c|c|c|c|c|}
\hline & \multicolumn{10}{|c|}{ Reference Data } & \multirow[b]{2}{*}{ Totals } & \multirow[b]{2}{*}{$\begin{array}{c}\text { User's } \\
\text { Accuracy }(\%)\end{array}$} \\
\hline & & $\begin{array}{c}\text { summer } \\
\text { deciduous } \\
\text { forest }\end{array}$ & $\begin{array}{l}\text { loblolly } \\
\text { pine }\end{array}$ & $\begin{array}{c}\text { autumn } \\
\text { deciduous }\end{array}$ & $\begin{array}{l}\text { green ag } \\
\text { field \#1 }\end{array}$ & $\begin{array}{l}\text { soil ag } \\
\text { field }\end{array}$ & $\begin{array}{l}\text { generic } \\
\text { road }\end{array}$ & $\begin{array}{l}\text { river } \\
\text { water }\end{array}$ & $\begin{array}{c}\text { shaded } \\
\text { vegetation }\end{array}$ & $\begin{array}{l}\text { grass } \\
\text { field }\end{array}$ & gravel & & \\
\hline \multirow{12}{*}{$\begin{array}{l}\text { Class } \\
\text { Map }\end{array}$} & $\begin{array}{l}\text { summer } \\
\text { deciduous } \\
\text { forest }\end{array}$ & 32493 & 17785 & 2436 & 6799 & 0 & 0 & 0 & 31 & 13 & 0 & 59557 & 54.56 \\
\hline & $\begin{array}{l}\text { loblolly } \\
\text { pine }\end{array}$ & 3580 & 74459 & 6830 & 951 & 0 & 4 & 0 & 12518 & 0 & 0 & 98342 & 75.71 \\
\hline & $\begin{array}{c}\text { autumn } \\
\text { deciduous }\end{array}$ & 334 & 0 & 7613 & 87 & 0 & 0 & 0 & 1 & 55 & 0 & 8090 & 94.10 \\
\hline & $\begin{array}{l}\text { green ag } \\
\text { field \#1 }\end{array}$ & 3097 & 1023 & 612 & 6247 & 13 & 82 & 0 & 23 & 395 & 1 & 11493 & 54.35 \\
\hline & $\begin{array}{l}\text { soil ag } \\
\text { field }\end{array}$ & 0 & 0 & 772 & 0 & 2254 & 20 & 0 & 0 & 2944 & 1 & 5991 & 37.62 \\
\hline & $\begin{array}{c}\text { generic } \\
\text { road }\end{array}$ & 0 & 0 & 149 & 0 & 153 & 2275 & 0 & 22 & 8 & 141 & 2748 & 82.79 \\
\hline & $\begin{array}{l}\text { river } \\
\text { water }\end{array}$ & 0 & 21 & 20 & 0 & 0 & 37 & 472 & 224 & 0 & 0 & 774 & 60.98 \\
\hline & $\begin{array}{c}\text { shaded } \\
\text { vegetation }\end{array}$ & 0 & 1392 & 88 & 0 & 0 & 19 & 127 & 2219 & 0 & 0 & 3845 & 57.71 \\
\hline & $\begin{array}{l}\text { grass } \\
\text { field }\end{array}$ & 1 & 0 & 4012 & 209 & 92 & 2 & 0 & 0 & 11965 & 1 & 16282 & 73.49 \\
\hline & gravel & 0 & 0 & 0 & 0 & 299 & 333 & 0 & 0 & 15 & 1641 & 2288 & 71.72 \\
\hline & Totals & 39505 & 94680 & 22532 & 14293 & 2811 & 2772 & 599 & 15038 & 15395 & 1785 & 209410 & \\
\hline & $\begin{array}{c}\text { Producer's } \\
\text { Accuracy (\%) }\end{array}$ & 82.25 & 78.64 & 33.79 & 43.71 & 80.18 & 82.07 & 78.80 & 14.76 & 77.72 & 91.93 & & $\begin{array}{c}\text { Overall } \\
\text { Accuracy }=67.64\end{array}$ \\
\hline
\end{tabular}

We compare our results with the ones obtained by [11] (See Table 6.3). Only the confusion matrix was available so we will make our comparison based on that. Though the overall accuracy is $86.46 \%$ in Table 6.3 which is higher than our score of $67.64 \%$. A quick judgment cannot be 


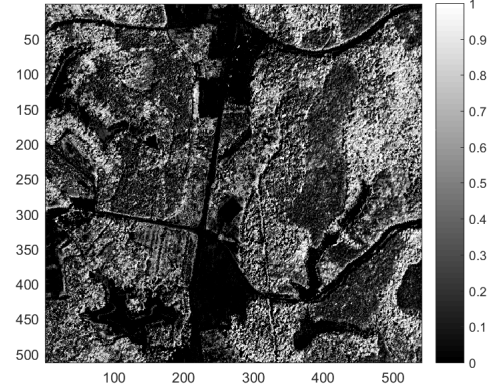

summer deciduous forest

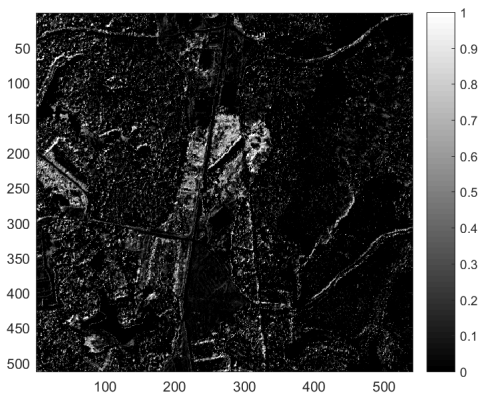

green ag field \#1

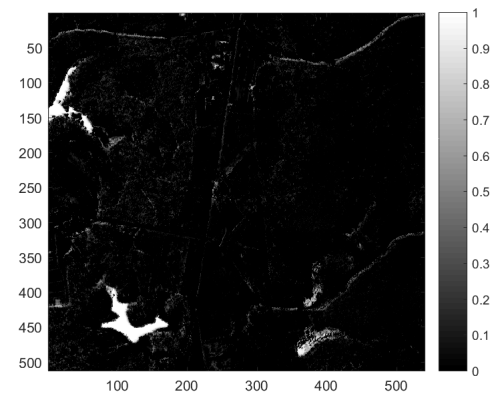

river water

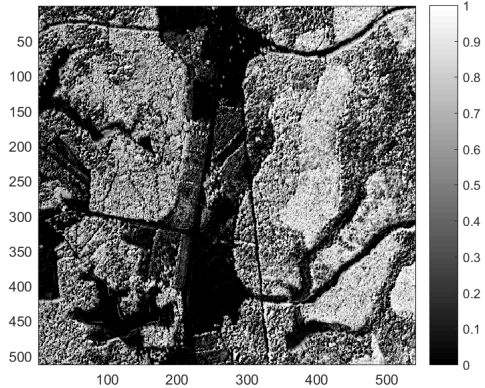

loblolly pine

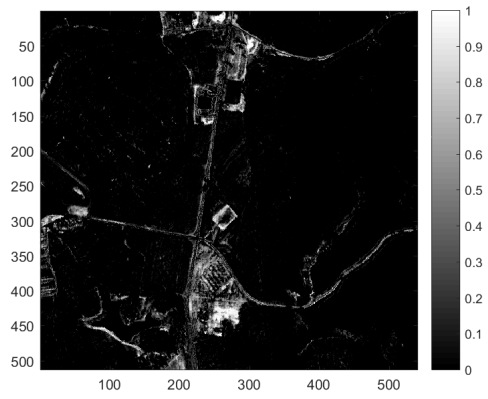

soil ag field

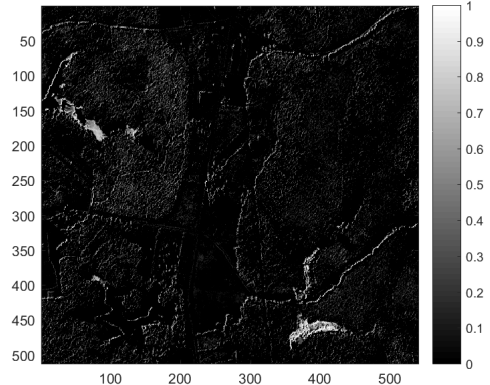

shaded vegetation

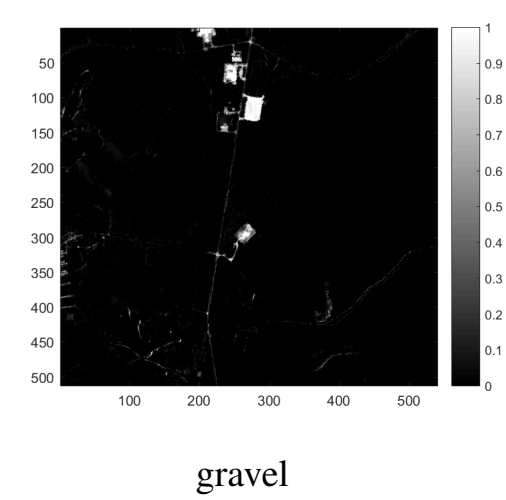

gravel

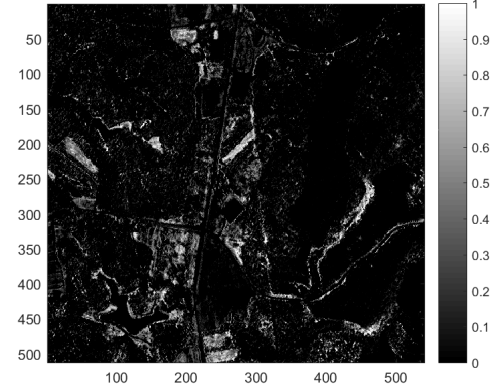

autumn deciduous

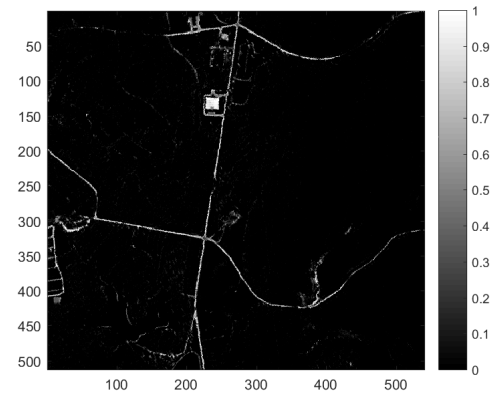

generic road

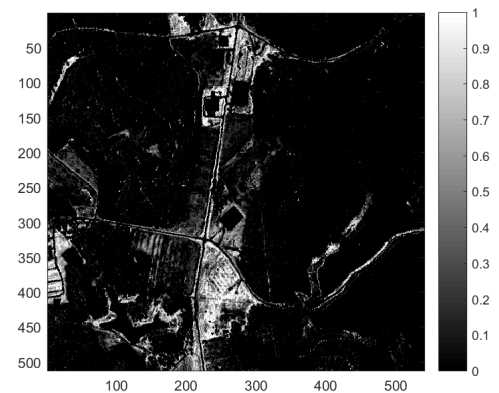

grass field

Figure 6.6: Information Abundance class maps extracted from the A.P. Hill Hyperspectral image using the proposed method 


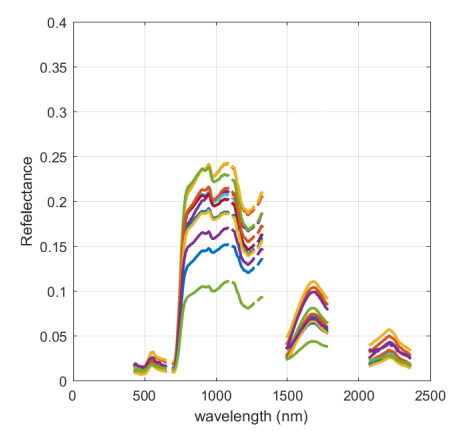

summer deciduous forest

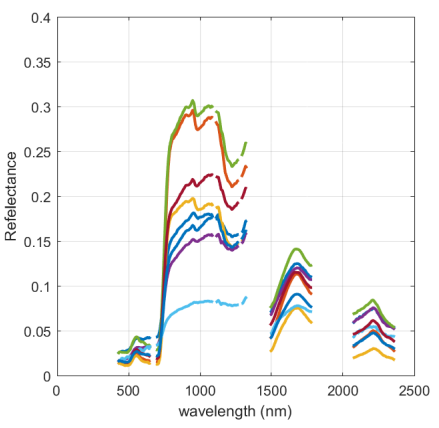

green ag field \#1

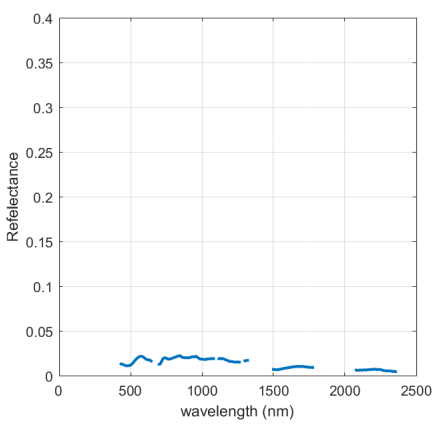

river water

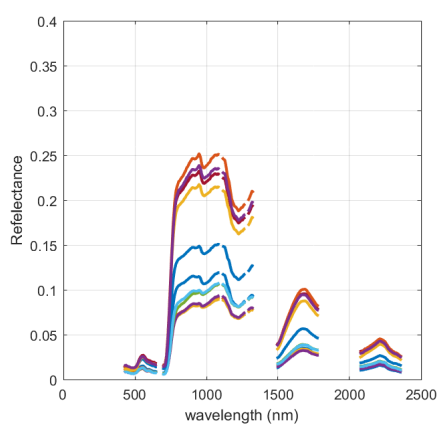

loblolly pine

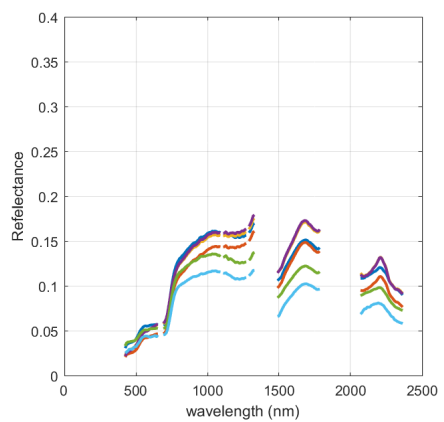

soil ag field

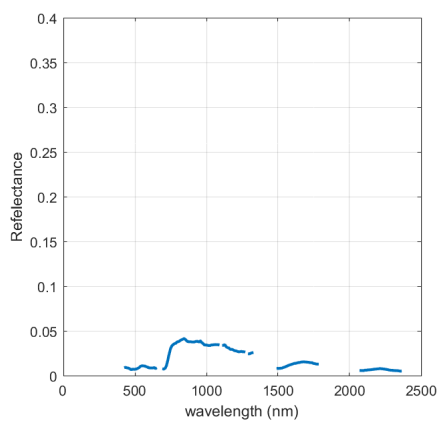

shaded vegetation

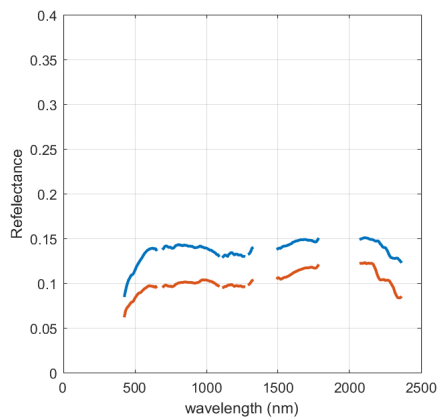

gravel

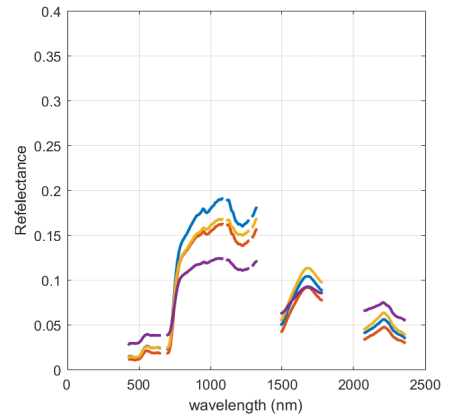

autumn deciduous

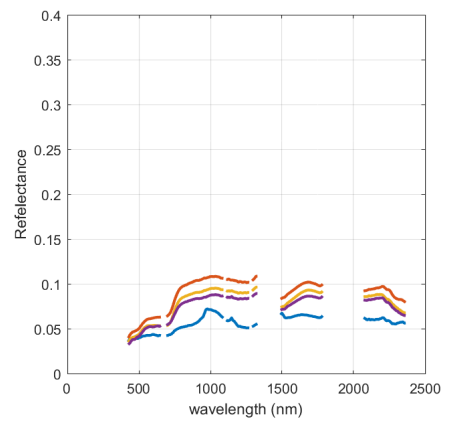

generic road

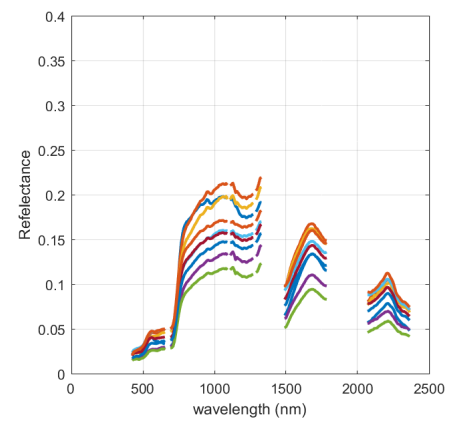

grass field

Figure 6.7: Information class spectral signatures extracted from the A.P. Hill Hyperspectral image using the proposed method 


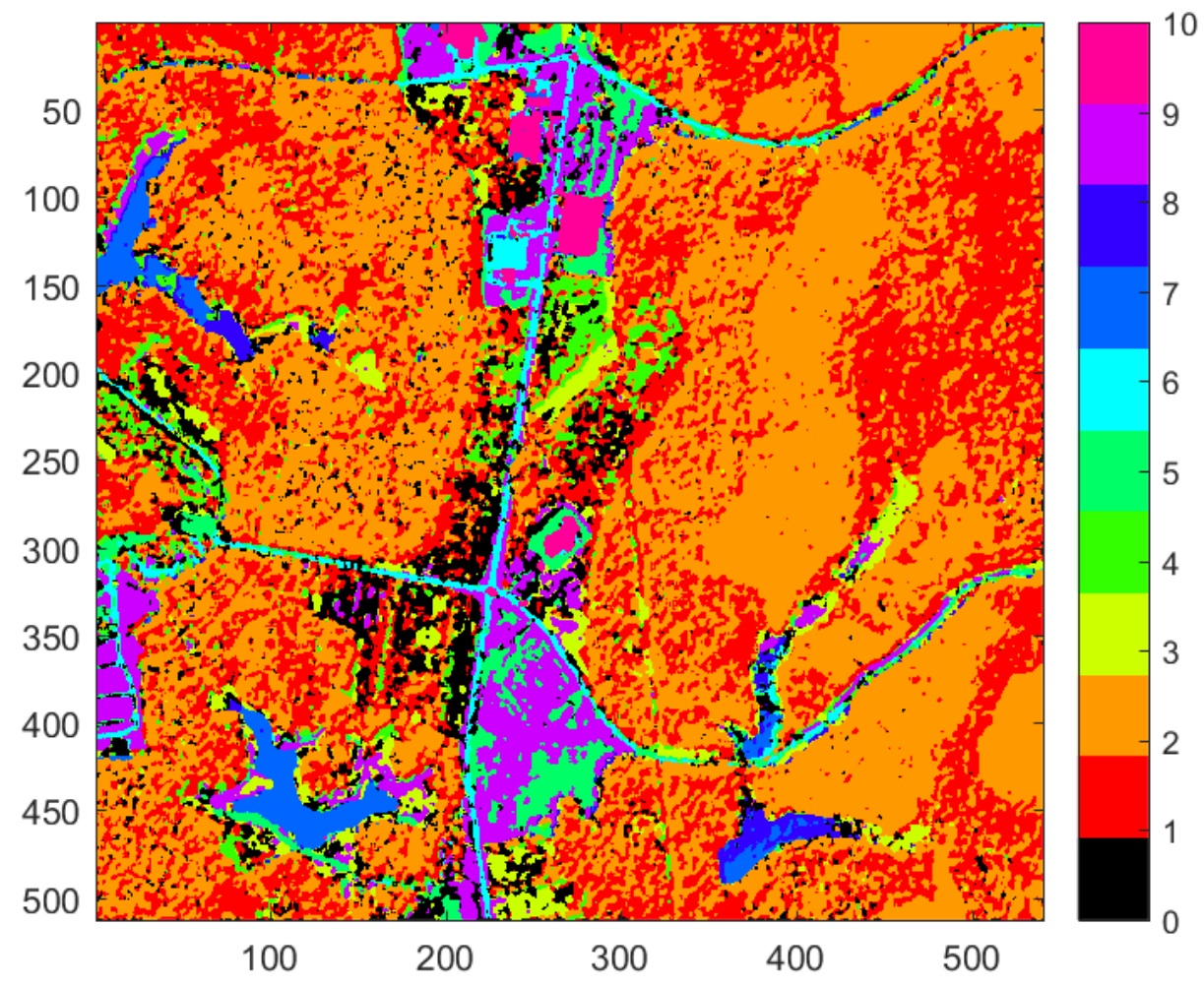

Figure 6.8: Generated class map using the proposed approach

made because the overall accuracy is just an average and does not reveal the distribution of error per class. We will focus our approach to the producer's accuracy between classes and compare the kappa coefficients of the two tables.

For the summer deciduous forest class, our approach scores values of $82.25 \%$ which is very close to the one obtained by [11] that was $(86920 / 100552)=86.44 \%$. The loblolly pine class has $78.64 \%$ producer's accuracy which is a little bit less than what is acquired in previous work which was $85.19 \%$. For the rest of classes, the results are close to each other (except for the shaded vegetation). In the end, the value of kappa for our approach was 51.98\% (Moderate Agreement) which is smaller to the one obtained by [11] of $82.56 \%$. 
Table 6.3: Confusion Matrix for A.P. Hill Image by [11]

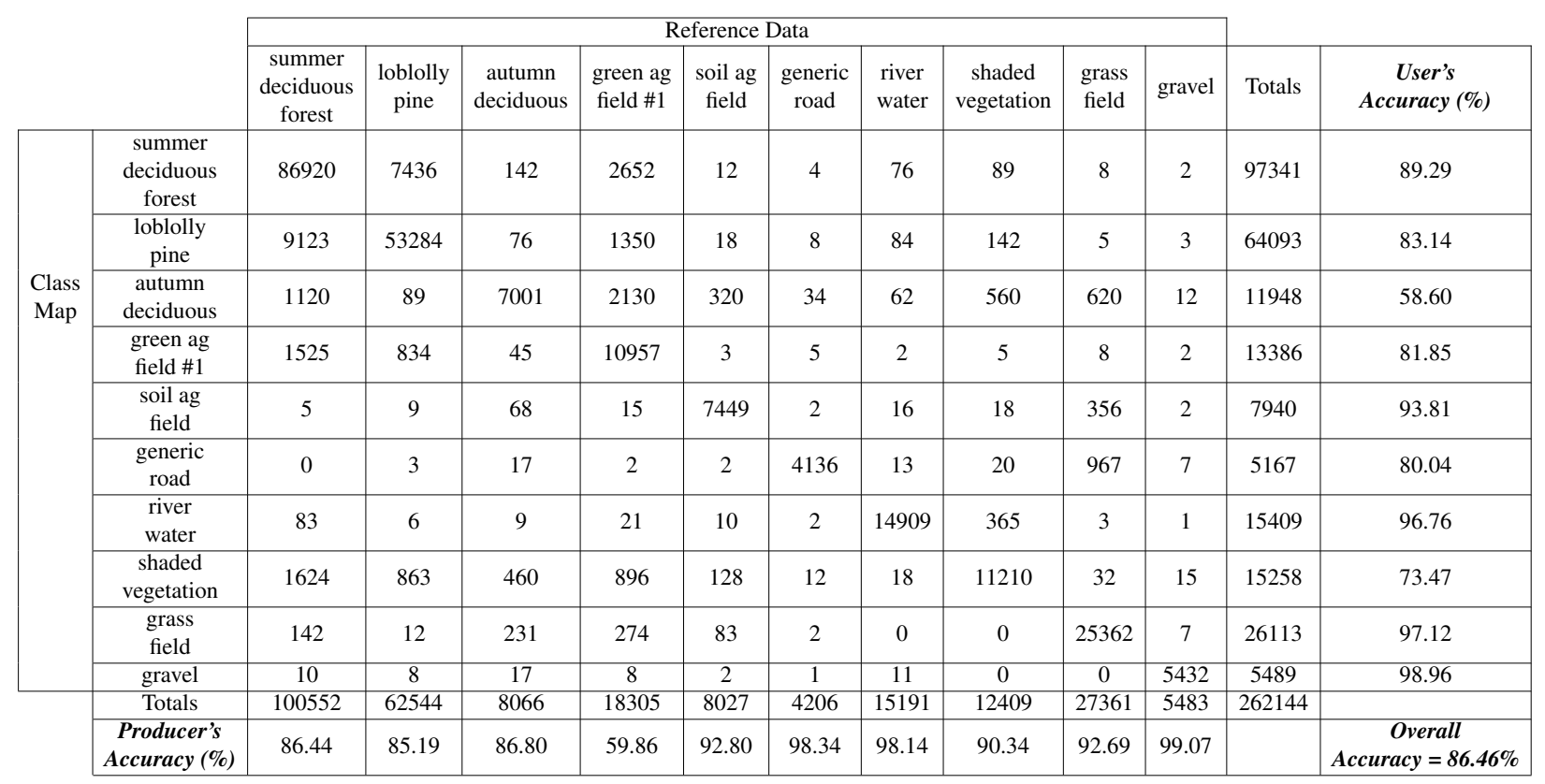

\subsection{Qualitative Evaluation with Existing High Resolution Image}

The class map of [8] is not very clear as mentioned in Section 6.2. Many class pixels do not follow any spatial uniformity and are scattered in the image. We tried to solve this problem in Section 6.3 by merging spectral classes that cover the same land cover, and filtering out individual pixels that do not follow any uniformity pattern.

Due to the dense mixing between vegetation classes that is hard to separate their spectral classes, we propose to use the concept of primary and secondary tree types. This concept has been introduced by [9] in order to give a better description of the scene. Figure 6.9 shows a high resolution version of the image where our region of study is shown in the cyan box. Figure 6.10 shows both primary and secondary tree classes mentioned before. In Figure 6.10(a), the image shows that the loblolly plantations in dark blue color as a primary tree class, however, there is no secondary class within the same region as shown in Figure 6.10(b). One can say that in this region, no mixing happens between loblolly plantations and other vegetation classes.

We will compare our results of abundance maps shown in Figure 6.6 to the classification maps 
in Figure 6.10. The loblolly Plantations in Figure 6.10(a) matches well with the loblolly pine class in Figure 6.6. In that particular region, no secondary trees are present and that matches our resulted abundance maps in Figure 6.6. The rest of the loblolly pine area appears as a primary class and is displayed in the khaki color (Virginia Pine) and dark yellow color (Loblolly Pine) of Figure 6.10(a) and also appears in brown (Virginia Pine) and yellow (Loblolly Pine) in the secondary class image in Figure 6.10(b).

The Deciduous Forest classes (Autumn and Summer) Matches the White Oak class marked with green color in Figure 6.10(a) and the Yellow Poplar class marked with golden color in Figure 6.10(b).

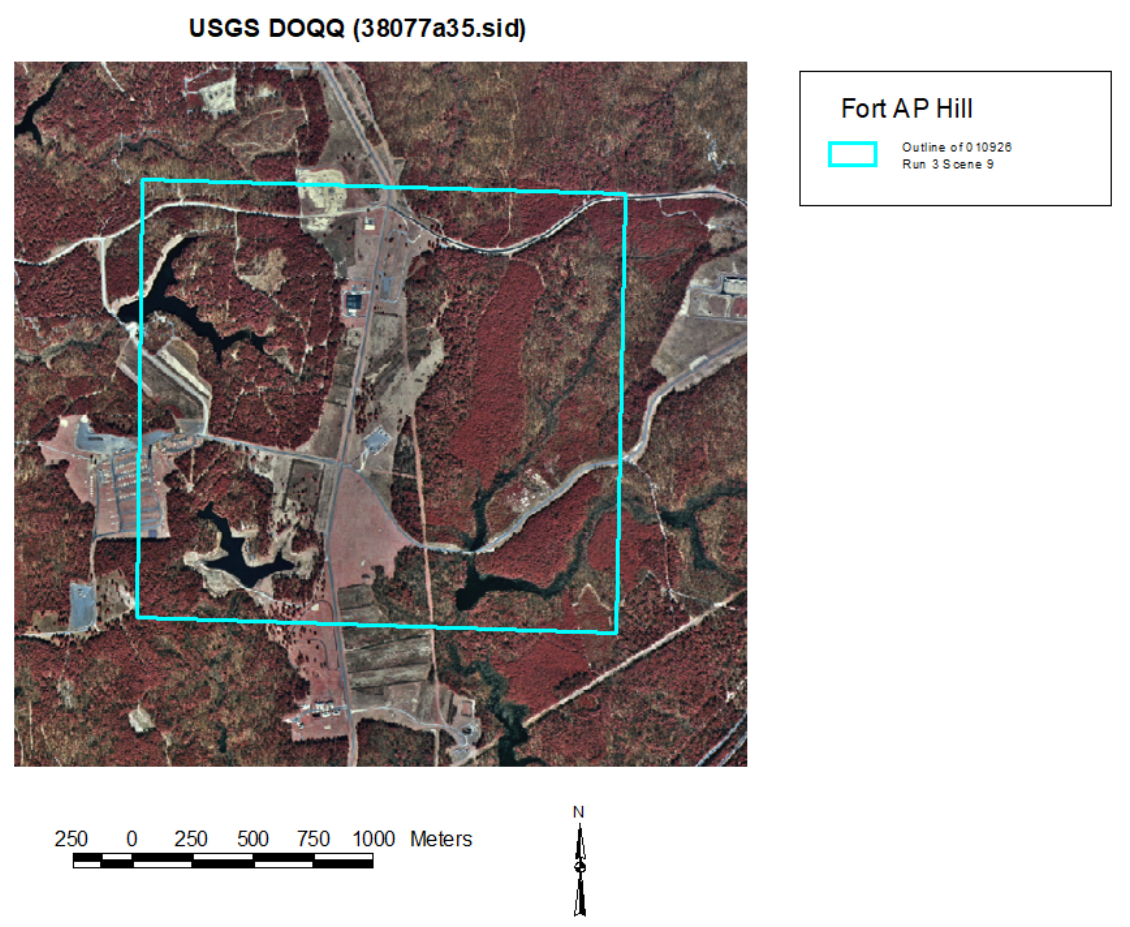

Figure 6.9: High Resolution image of Fort A.P. Hill

\subsection{Summary}

The same approach has been applied on a complex hyperspectral scene (Fort A.P. Hill), using similar analysis as in Chapter 5. We used quantitative analysis (based on the confusion matrix) to 


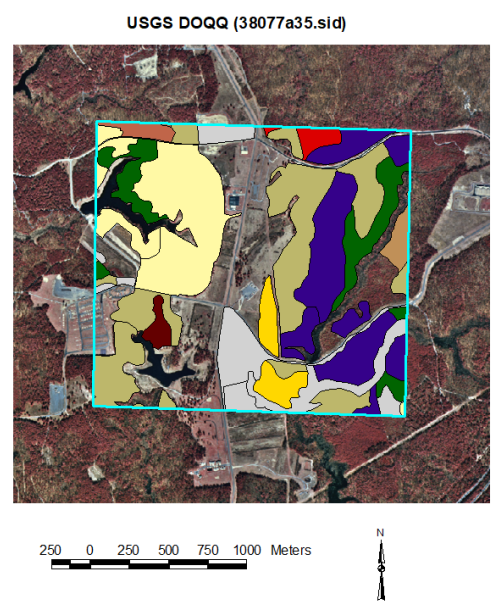

(a)
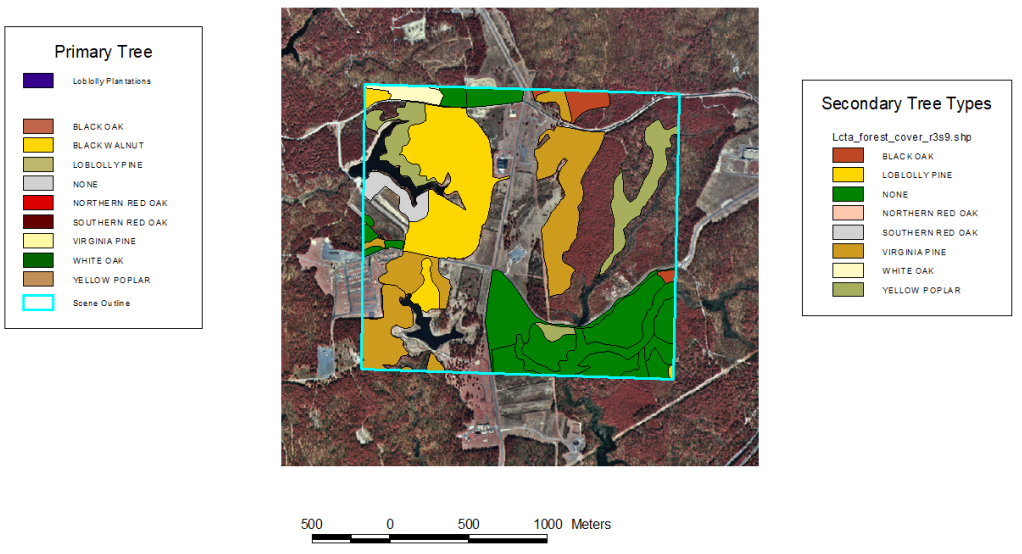

(b)

Figure 6.10: A.P. Hill vegetation maps [9]: (a) Primary Trees; (b) Secondary Trees

evaluate the performance of our algorithm. The quantitative approach was based on the confusion matrix between the new reference data class map (where we combined similar land cover classes together to reduce the complexity of the scene) and the generated class map from our approach. We mainly focused our evaluation based on the value of Kappa coefficient that was $51.98 \%$, a relatively low value. But considering the image complexity of the image, the value is accepted.

We then compare our results with previous published work that was based on the quadtree method by [11], the overall agreement of the quadtree approach shows a high percentage when compared to our approach. The Kappa scored was also very high (almost 82\%). However, the results obtained were not documented well and no class map was produced to validate the its performance.

In Section 6.5, we evaluate our method by visual comparison with Primary and Secondary tree classes available from [9]. Our results (the vegetation classes) matches well with the available vegetation maps. 


\section{Chapter 7}

\section{CONCLUSIONS AND FUTURE WORK}

\subsection{Conclusions}

Unmixing analysis of hyperspectral imagery is usually performed by spectral techniques which do not employ the spatial information of the images. Recently, spatial-spectral algorithms have been developed. Most of these algorithms incorporate the spatial information in the unmixing analysis by using spatial kernels. However, this introduces dependence on the size of neighborhood. As proposed in [5], a spatial-spectral algorithm that avoid the use of spatial kernels using quadtree segmentation to look for spectrally uniform regions. However, this method divides the image into rectangular tiles and won't reach spectrally homogeneous regions until large number of levels is processed to produce the segmentation. In addition, it requires the estimation of the number of endmembers like most of the existing unmixing algorithms.

This work developed a new spatial-spectral approach for unmixing analysis using Superpixel segmentation for low dimensional representation combined with quadtree segmentation for regional endmember extraction using SVDSS. A low dimensional image representation is obtained using superpixel segmentation where each superpixel is represented by its mean spectra. Regional analysis is then performed using the quadtree segmentation based on the Shannon entropy. Endmembers are extracted from each region and combined using clustering into endmember classes based on their spectral angle distance. Abundances are then calculated globally using the extracted endmembers, abundance maps are grouped based on their corresponding endmember classes.

Experimental analysis of the unsupervised unmixing approach was conducted using real hyperpectral imagery from HyDICE and ROSIS sensors. It is important to recognize that there are great limitations to perform quantitative assessment of unmixing results. Most of the time, only classification maps and spectral libraries are available for hyperspectral dataset. Even the spectral libraries are usually laboratory spectra. Thus, the assessment methodology was based on the 
confusion matrix comparing the proposed approach and published classification maps for a quantitative evaluation. First, the assessment methodology uses a qualitative comparison between the estimated abundances and the information classes on the scene. It is assumed that the classification maps provide information about the distribution of materials, and one or more spectral endmember classes can be related to the information classes within the classification map. Unmixing results for Urban and Pavia University were related to several materials in the reference classification maps showing consistency among spectral endmember and reference spectra. Once the relation between spectral endmember classes and information classes was established, the assessment methodology generates a classification map using the estimated abundances and a majority vote criterion. The classification maps obtained for Urban and Pavia show good correspondence with the published classification maps. The quantitative assessment is performed using four statistics: Producer's Accuracy, User's Accuracy, Overall accuracy and Kappa statistic. The proposed unmixing approach obtained good results for most of the Urban and Pavia classes. The focus was on the Kappa statistic value which was over $80 \%$ for both data sets indicating an almost perfect agreement.

The proposed approach (LD+QT) was compared with three other unmixing scenarios: (i) Full image segmented using quadtree approach (Full+QT); (ii) global unmixing of the full image with no spatial segmentation (Full Global); and (iii) global analysis of the SP image without any spatial segmentation (LD Global). All methods were applied to the Urban data set. The Full+QT approach was selected since it is recently used by [5] and it showed very good unmixing results. The Full global method is conducted since it is the classical unmixing approach. The LD Global approach is conducted to see how the regional segmentation of the LD image was an essential step for improving the unmixing analysis. Our method showed the best results in terms of overall accuracy and Kappa statistic which was $85.01 \%$. Next to it by a little bit was the Full+QT result who scored a $83.03 \%$. Both regional based results are close, however, our approach scored the best result in a considerably shorter time (almost less by $80 \%$ of the time). Also, the extracted endmembers using our approach in Figure 5.12 look clean and match the reference data when compared to the ones in Figure 5.34. The global results with no regional segmentation didn't perform well when compared 
to the previous two. This shows how essential the regional segmentation in the unmixing analysis in general.

The proposed approach was tested on AVIRIS Fort A.P. Hill. The image is mostly a forest area with a mix of deciduous and coniferous species. The image is considered a hard one for unmixing since most materials are heavily mixed together and spectral signatures in many classes are very similar. We first combined similar endmember classes that describe the same land cover, which reduced the number of classes to 10 instead of 14 . The results obtained were not as good as the ones obtained from Urban or Pavia. Quantitative analysis are conducted using the confusion matrix in Table 6.2 shows that the autumn deciduous class was badly captured using our approach and most of its pixels were labeled as loblolly pine and green field. The shaded vegetation class also has most of its pixels labeled as loblolly pine. This actually happens due to the similarity of these materials (all are vegetation classes). We then compared our results visually with a classification map [9] that describes the vegetation classes as primary and secondary classes. Our results somehow follows the primary and secondary vegetation maps.

The main contribution of this work was the integration of superpixel segmentation, dimensionality reduction and SVDSS for the development of a new approach for the unmixing analysis of hyperspectral imagery. Many of these techniques are widely used in image processing. But, there is no previous work that uses superpixel segmentation and SVDSS for the unmixing analysis. Superpixel segmentation for dimensionality reduction followed with regional segmentation using quadtree allow the integration of spatial information in the unmixing analysis. The proposed unmixing approach provides useful information (spectral endmember classes and abundances) but these products still require further interpretation and analysis by experts.

\subsection{Future Work}

Some challenges are still there and need to be solved, the main one is to find the optimal region size in the SLIC algorithm. Having a large value for region size will reduce the chance to extract endmembers with low variance. On the other side, having a small value for the region size will 
reduce the number of pixels in each superpixel and that may result in reducing the convexity of the cloud represented by the superpixel pixels and that may affect the endmember extraction process .

The second area of study is look for another method to represent the SLIC superpixels. In our research, we used the spectral mean of signatures at each superpixel as a representative to it. However, we assumed resulting superpixels to be spectrally uniform, that might not be a correct assumption and the centroid may not be a good representation. One suggestion is to exploit the superpixel structure rather than just using the mean spectra, this might open the door to a better extraction of endmembers from the image and may enhance the unmixing process.

The third area of study is to find a more automated way to find the threshold for the endmember clustering algorithms. We used the angle distance to form endmember spectral classes with a threshold of 0.005 in the Urban and Pavis data sets. While we used the value of 0.001 on the A.P. Hill image. The threshold value could be a tuned parameter that depends on the type of materials in the scene. 


\section{REFERENCES}

[1] S. Centre for Remote Imaging and P. (CRISP), "What is remote sensing?." https : //crisp. nus.edu.sg/ research/tutorial/intro.htm. accessed Jan 2018.

[2] N. Keshava and J. F. Mustard, "Spectral unmixing," IEEE signal processing magazine, vol. 19, no. 1, pp. 44-57, 2002.

[3] A. Plaza, P. Martínez, R. Pérez, and J. Plaza, "Spatial/spectral endmember extraction by multidimensional morphological operations," IEEE transactions on geoscience and remote sensing, vol. 40, no. 9, pp. 2025-2041, 2002.

[4] J. M. Duarte-Carvajalino, G. Sapiro, M. Vélez-Reyes, and P. E. Castillo, "Multiscale representation and segmentation of hyperspectral imagery using geometric partial differential equations and algebraic multigrid methods," IEEE Transactions on Geoscience and Remote Sensing, vol. 46, no. 8, pp. 2418-2434, 2008.

[5] M. A. Goenaga-Jimenez and M. Vélez-Reyes, "Integrating spatial information in unmixing using the nonnegative matrix factorization," in Algorithms and Technologies for Multispectral, Hyperspectral, and Ultraspectral Imagery XX, vol. 9088, p. 908811, International Society for Optics and Photonics, 2014.

[6] D. A. Landgrebe, Signal theory methods in multispectral remote sensing, vol. 29. John Wiley \& Sons, 2005.

[7] F. Zhu, Y. Wang, B. Fan, G. Meng, and C. Pan, "Effective spectral unmixing via robust representation and learning-based sparsity," CoRR, vol. abs/1409.0685, 2014.

[8] J. J. Cipar, R. Lockwood, T. Cooley, and P. Grigsby, "Background spectral library for fort ap hill, virginia," in Remote Sensing and Modeling of Ecosystems for Sustainability, vol. 5544, pp. 35-47, International Society for Optics and Photonics, 2004.

[9] M. A. Glennon, “Landcover types from afrl/vsbyh gis database.” Technical Report, 2004.

[10] J. R. Landis and G. G. Koch, "The measurement of observer agreement for categorical data," biometrics, pp. 159-174, 1977.

[11] M. A. Goenaga-Jimenez, Spatially Adaptive Constrained Non Negative Matrix Factorization for Hyperspectral Unmixing. PhD thesis, University of Puerto Rico - MAYAGUEZ CAMPUS, 2014.

[12] A. K. Tilling, G. O’Leary, J. Ferwerda, S. Jones, G. Fitzgerald, and R. Belford, "Remote sensing to detect nitrogen and water stress in wheat," The Australian Society of Agronomy, vol. 17, 2006.

[13] M. Burgstaller and M. Groinig, "Spectral imaging sorts 'sugar-end' defects." http://www .vision-systems . com/articles/print/volume-17/issue-2/ features/spectral-imaging-sorts-sugar-end-defects.html. Feb 2012, accessed Jan 2018. 
[14] A. Shahidi, S. Patel, J. Flanagan, and C. Hudson, "Regional variation in human retinal vessel oxygen saturation," Experimental eye research, vol. 113, pp. 143-147, 2013.

[15] https://directory.eoportal.org/web/eoportal/airborne-sensors/aviris . accessed Jan 2018.

[16] B. Kunkel, F. Blechinger, R. Lutz, R. Doerffer, H. van der Piepen, and M. Schroder, "Rosis (reflective optics system imaging spectrometer) a candidate instrument for polar platform missions," in Optoelectronic technologies for remote sensing from space, vol. 868, pp. 134$141,1988$.

[17] USGS, "Sensors - hyperion." https://eo1.usgs.gov/sensors/hyperion. accessed Jan 2018.

[18] NASA, "Hyspiri mission study." https://hyspiri.jpl.nasa.gov/. accessed Jan 2018.

[19] M. Vélez-Reyes and S. Rosario, "Solving adundance estimation in hyperspectral unmixing as a least distance problem," in Geoscience and Remote Sensing Symposium, 2004. IGARSS'04. Proceedings. 2004 IEEE International, vol. 5, pp. 3276-3278, IEEE, 2004.

[20] A. Zare and P. Gader, "Pce: Piecewise convex endmember detection," IEEE Transactions on Geoscience and Remote Sensing, vol. 48, no. 6, pp. 2620-2632, 2010.

[21] M. Parente and A. Plaza, "Survey of geometric and statistical unmixing algorithms for hyperspectral images," in Hyperspectral Image and Signal Processing: Evolution in Remote Sensing (WHISPERS), 2010 2nd Workshop on, pp. 1-4, IEEE, 2010.

[22] N. Keshava, "A survey of spectral unmixing algorithms," Lincoln laboratory journal, vol. 14, no. 1, pp. 55-78, 2003.

[23] K. Wong, Q. Zhang, J. Reilly, and P. Yip, "A new criterion for the determination of the number of signals in high-resolution array processing," in Acoustics, Speech, and Signal Processing, 1988. ICASSP-88., 1988 International Conference on, pp. 2769-2772, IEEE, 1988.

[24] J. W. Boardman, "Automating spectral unmixing of aviris data using convex geometry concepts," 1993.

[25] A. Ifarraguerri and C.-I. Chang, "Multispectral and hyperspectral image analysis with convex cones," IEEE transactions on geoscience and remote sensing, vol. 37, no. 2, pp. 756-770, 1999.

[26] M. E. Winter, "N-findr: An algorithm for fast autonomous spectral end-member determination in hyperspectral data," in Imaging Spectrometry V, vol. 3753, pp. 266-276, International Society for Optics and Photonics, 1999.

[27] J. M. Nascimento and J. M. Dias, "Vertex component analysis: A fast algorithm to unmix hyperspectral data," IEEE transactions on Geoscience and Remote Sensing, vol. 43, no. 4, pp. 898-910, 2005. 
[28] D. D. Lee and H. S. Seung, "Learning the parts of objects by non-negative matrix factorization," Nature, vol. 401, no. 6755, p. 788, 1999.

[29] S. Jia and Y. Qian, "Constrained nonnegative matrix factorization for hyperspectral unmixing," IEEE Transactions on Geoscience and Remote Sensing, vol. 47, no. 1, pp. 161-173, 2009.

[30] Y. M. Masalmah and M. Veléz-Reyes, "A full algorithm to compute the constrained positive matrix factorization and its application in unsupervised unmixing of hyperspectral imagery," in Algorithms and Technologies for Multispectral, Hyperspectral, and Ultraspectral Imagery XIV, vol. 6966, p. 69661C, International Society for Optics and Photonics, 2008.

[31] A. D. Stocker and A. P. Schaum, "Application of stochastic mixing models to hyperspectral detection problems," in Algorithms for Multispectral and Hyperspectral Imagery III, vol. 3071, pp. 47-61, International Society for Optics and Photonics, 1997.

[32] J. M. Nascimento and J. M. Bioucas-Dias, "Hyperspectral unmixing algorithm via dependent component analysis," in Geoscience and Remote Sensing Symposium, 2007. IGARSS 2007. IEEE International, pp. 4033-4036, IEEE, 2007.

[33] A. Zare and P. D. Gader, "Endmember detection using the dirichlet process," in Pattern Recognition, 2008. ICPR 2008. 19th International Conference on, pp. 1-4, IEEE, 2008.

[34] O. Eches, N. Dobigeon, and J.-Y. Tourneret, "Estimating the number of endmembers in hyperspectral images using the normal compositional model and a hierarchical bayesian algorithm," IEEE Journal of Selected Topics in Signal Processing, vol. 4, no. 3, pp. 582-591, 2010 .

[35] D. M. Rogge, B. Rivard, J. Zhang, A. Sanchez, J. Harris, and J. Feng, "Integration of spatialspectral information for the improved extraction of endmembers," Remote Sensing of Environment, vol. 110, no. 3, pp. 287-303, 2007.

[36] K. Canham, A. Schlamm, A. Ziemann, B. Basener, and D. Messinger, "Spatially adaptive hyperspectral unmixing," IEEE Transactions on Geoscience and Remote Sensing, vol. 49, no. 11, pp. 4248-4262, 2011.

[37] K. Lee, A subpixel scale target detection algorithm for hyperspectral imagery. PhD thesis, PhD dissertation, Rochester Institute of Technology, 54 Lomb Memorial Drive, Rochester, NY, 2003.

[38] M. C. Torres-Madronero and M. Velez-Reyes, "Unsupervised unmixing analysis based on multiscale representation," in Algorithms and Technologies for Multispectral, Hyperspectral, and Ultraspectral Imagery XVIII, vol. 8390, p. 83901O, International Society for Optics and Photonics, 2012.

[39] M. W. Palmer, P. G. Earls, B. W. Hoagland, P. S. White, and T. Wohlgemuth, "Quantitative tools for perfecting species lists," Environmetrics, vol. 13, no. 2, pp. 121-137, 2002. 
[40] J. Shlens, "A tutorial on principal component analysis," arXiv preprint arXiv:1404.1100, 2014.

[41] C.-I. Chang and Q. Du, "Estimation of number of spectrally distinct signal sources in hyperspectral imagery," IEEE Transactions on geoscience and remote sensing, vol. 42, no. 3, pp. 608-619, 2004.

[42] H. Akaike, "Information theory and an extension of the maximum likelihood principle," in Breakthroughs in statistics, pp. 610-624, Springer, 1992.

[43] J. Rissanen, "Modeling by shortest data description," Automatica, vol. 14, no. 5, pp. 465-471, 1978.

[44] M. Wax and T. Kailath, "Detection of signals by information theoretic criteria," IEEE Transactions on Acoustics, Speech, and Signal Processing, vol. 33, no. 2, pp. 387-392, 1985.

[45] C.-C. Wu, W. Liu, and C.-I. Chang, "Exploration of methods for estimation of number of endmembers in hyperspectral imagery," in Chemical and Biological Sensors for Industrial and Environmental Monitoring II, vol. 6378, p. 63781C, International Society for Optics and Photonics, 2006.

[46] P. Bajorski, "Does virtual dimensionality work in hyperspectral images?," in Algorithms and Technologies for Multispectral, Hyperspectral, and Ultraspectral Imagery XV, vol. 7334, p. 73341J, International Society for Optics and Photonics, 2009.

[47] J. Van Den Hof, "Realization of positive linear systems," Linear Algebra and its Applications, vol. 256, pp. 287-308, 1997.

[48] J. M. Bioucas-Dias and J. M. Nascimento, "Hyperspectral subspace identification," IEEE Transactions on Geoscience and Remote Sensing, vol. 46, no. 8, pp. 2435-2445, 2008.

[49] D. Messinger, A. Ziemann, A. Schlamm, and B. Basener, "Spectral image complexity estimated through local convex hull volume," in Hyperspectral Image and Signal Processing: Evolution in Remote Sensing (WHISPERS), 2010 2nd Workshop on, pp. 1-4, IEEE, 2010.

[50] D. W. Messinger, A. K. Ziemann, A. Schlamm, and W. Basener, "Metrics of spectral image complexity with application to large area search," Optical Engineering, vol. 51, no. 3, p. 036201, 2012.

[51] P. Suetin, A. I. Kostrikin, and Y. I. Manin, Linear algebra and geometry, vol. 1. CRC Press, 1997.

[52] S. Rosario-Torres and M. Vélez-Reyes, "An algorithm for fully constrained abundance estimation in hyperspectral unmixing," in Algorithms and Technologies for Multispectral, Hyperspectral, and Ultraspectral Imagery XI, vol. 5806, pp. 711-720, International Society for Optics and Photonics, 2005.

[53] A. Castrodad, Z. Xing, J. Greer, E. Bosch, L. Carin, and G. Sapiro, "Discriminative sparse representations in hyperspectral imagery," in Image Processing (ICIP), 2010 17th IEEE International Conference on, pp. 1313-1316, IEEE, 2010. 
[54] Y. Chen, N. M. Nasrabadi, and T. D. Tran, "Sparse representation for target detection in hyperspectral imagery," IEEE Journal of Selected Topics in Signal Processing, vol. 5, no. 3, pp. 629-640, 2011.

[55] M.-D. Iordache, J. M. Bioucas-Dias, and A. Plaza, "Sparse unmixing of hyperspectral data," IEEE Transactions on Geoscience and Remote Sensing, vol. 49, no. 6, pp. 2014-2039, 2011.

[56] D. R. Thompson, L. Mandrake, M. S. Gilmore, and R. Castano, "Superpixel endmember detection," IEEE Transactions on Geoscience and Remote Sensing, vol. 48, no. 11, pp. 40234033, 2010.

[57] A. M. Saranathan and M. Parente, "Uniformity-based superpixel segmentation of hyperspectral images," IEEE Transactions on Geoscience and Remote Sensing, vol. 54, no. 3, pp. 14191430, 2016.

[58] R. Achanta, A. Shaji, K. Smith, A. Lucchi, P. Fua, and S. Süsstrunk, "Slic superpixels compared to state-of-the-art superpixel methods," IEEE transactions on pattern analysis and machine intelligence, vol. 34, no. 11, pp. 2274-2282, 2012.

[59] S. X. Yu, "Segmentation induced by scale invariance," in Computer Vision and Pattern Recognition, 2005. CVPR 2005. IEEE Computer Society Conference on, vol. 1, pp. 444-451, IEEE, 2005.

[60] D. Comaniciu and P. Meer, "Mean shift: A robust approach toward feature space analysis," IEEE Transactions on pattern analysis and machine intelligence, vol. 24, no. 5, pp. 603-619, 2002.

[61] L. Vincent and P. Soille, "Watersheds in digital spaces: an efficient algorithm based on immersion simulations," IEEE Transactions on Pattern Analysis \& Machine Intelligence, no. 6, pp. 583-598, 1991.

[62] X. Zhang, S. E. Chew, Z. Xu, and N. D. Cahill, "Slic superpixels for efficient graph-based dimensionality reduction of hyperspectral imagery," in Algorithms and Technologies for Multispectral, Hyperspectral, and Ultraspectral Imagery XXI, vol. 9472, p. 947209, International Society for Optics and Photonics, 2015.

[63] M. A. Goenaga-Jimenez and M. Vélez-Reyes, "Comparing quadtree region partitioning metrics for hyperspectral unmixing," in Algorithms and Technologies for Multispectral, Hyperspectral, and Ultraspectral Imagery XIX, vol. 8743, p. 87430Z, International Society for Optics and Photonics, 2013.

[64] A. Halevy, Extensions of laplacian eigenmaps for manifold learning. PhD thesis, 2011.

[65] J. Benedetto, W. Czaja, J. Dobrosotskaya, T. Doster, K. Duke, and D. Gillis, "Semisupervised learning of heterogeneous data in remote sensing imagery," in Independent Component Analyses, Compressive Sampling, Wavelets, Neural Net, Biosystems, and Nanoengineering $X$, vol. 8401, p. 840104, International Society for Optics and Photonics, 2012. 
[66] B. Somers, M. Zortea, A. Plaza, and G. P. Asner, "Automated extraction of image-based endmember bundles for improved spectral unmixing," IEEE Journal of Selected Topics in Applied Earth Observations and Remote Sensing, vol. 5, no. 2, pp. 396-408, 2012.

[67] G. Camps-Valls, T. V. B. Marsheva, and D. Zhou, "Semi-supervised graph-based hyperspectral image classification," IEEE Transactions on Geoscience and Remote Sensing, vol. 45, no. 10, pp. 3044-3054, 2007.

[68] D. T. Anderson and A. Zare, "Spectral unmixing cluster validity index for multiple sets of endmembers," IEEE Journal of Selected Topics in Applied Earth Observations and Remote Sensing, vol. 5, no. 4, pp. 1282-1295, 2012.

[69] R. A. Mercovich, J. Albano, and D. Messinger, "Techniques for the graph representation of spectral imagery," in Hyperspectral Image and Signal Processing: Evolution in Remote Sensing (WHISPERS), 2011 3rd Workshop on, pp. 1-4, IEEE, 2011.

[70] Y. Tarabalka, J. A. Benediktsson, and J. Chanussot, "Spectral-spatial classification of hyperspectral imagery based on partitional clustering techniques," IEEE Transactions on Geoscience and Remote Sensing, vol. 47, no. 8, pp. 2973-2987, 2009.

[71] A. K. Jain and R. C. Dubes, "Algorithms for clustering data," 1988.

[72] A. K. Jain, M. N. Murty, and P. J. Flynn, "Data clustering: a review," ACM computing surveys (CSUR), vol. 31, no. 3, pp. 264-323, 1999.

[73] R. Xu and D. Wunsch, "Survey of clustering algorithms," IEEE Transactions on neural networks, vol. 16, no. 3, pp. 645-678, 2005.

[74] F. A. Kruse, A. Lefkoff, J. Boardman, K. Heidebrecht, A. Shapiro, P. Barloon, and A. Goetz, "The spectral image processing system (sips) - interactive visualization and analysis of imaging spectrometer data," Remote sensing of environment, vol. 44, no. 2-3, pp. 145-163, 1993.

[75] F. van der Meero and W. Bakker, "Cross correlogram spectral matching: application to surface mineralogical mapping by using aviris data from cuprite, nevada," Remote Sensing of Environment, vol. 61, no. 3, pp. 371-382, 1997.

[76] C.-I. Chang, "Spectral information divergence for hyperspectral image analysis," in Geoscience and Remote Sensing Symposium, 1999. IGARSS'99 Proceedings. IEEE 1999 International, vol. 1, pp. 509-511, IEEE, 1999.

[77] Mathworks, "Statistics and machine learning toolbox ${ }^{\mathrm{TM}}$ user's guide." https://www. mathworks.com/help/pdf_doc/stats/stats.pdf. 2017, accessed Jan 2018.

[78] U. Von Luxburg, "A tutorial on spectral clustering," Statistics and computing, vol. 17, no. 4, pp. 395-416, 2007.

[79] J. Yi and M. Velez-Reyes, "Dimensionality reduction using superpixel segmentation for hyperspectral unmixing using the cnmf," in Algorithms and Technologies for Multispectral, Hyperspectral, and Ultraspectral Imagery XXIII, vol. 10198, p. 101981H, International Society for Optics and Photonics, 2017. 
[80] M. Aldeghlawi and M. Vélez-Reyes, "Column subset selection methods for endmember extraction in hyperspectral unmixing," in Algorithms and Technologies for Multispectral, Hyperspectral and Ultraspectral Imaging XXIV, vol. 10644, International Society for Optics and Photonics, 2018.

[81] M. Velez-Reyes and L. O. Jimenez, "Subset selection analysis for the reduction of hyperspectral imagery," in Geoscience and Remote Sensing Symposium Proceedings, 1998. IGARSS'98. 1998 IEEE International, vol. 3, pp. 1577-1581, IEEE, 1998.

[82] I. T. Jolliffe, "Principal component analysis and factor analysis," in Principal component analysis, pp. 44-47, Springer, 1986.

[83] J. Li, J. M. Bioucas-Dias, and A. Plaza, "Collaborative nonnegative matrix factorization for remotely sensed hyperspectral unmixing," in Geoscience and Remote Sensing Symposium (IGARSS), 2012 IEEE International, pp. 3078-3081, IEEE, 2012.

[84] A. Vedaldi and B. Fulkerson, "Slic superpixels." http://www.vlfeat.org/overview/ slic.html. accessed Jan 2018.

[85] F. Zhu, "Hyperspectral unmixing: Ground truth labeling, datasets, benchmark performances and survey,"

[86] A. Plaza, P. Martínez, R. Perez, and J. Plaza, "A comparative analysis of endmember extraction algorithms using aviris hyperspectral imagery," in Summaries of the 11th JPL Airborne Earth Science Workshop, p. 2002, JPL, 2002.

[87] J. Cohen, "A coefficient of agreement for nominal scales," Educational and psychological measurement, vol. 20, no. 1, pp. 37-46, 1960.

[88] S. V. Stehman, "Selecting and interpreting measures of thematic classification accuracy," Remote sensing of Environment, vol. 62, no. 1, pp. 77-89, 1997. 


\section{CURRICULUM VITA}

Mohammed Q. Alkhatib finished his bachelor degree in Telecommunications engineering in June, 2008 from the Hijjawi faculty for science and engineering, Yarmouk University, Irbid, Jordan with a very good overall percentage of $79 \%$.

In spring 2011, he earned his Master's degree Electrical Engineering from the University of Texas at El Paso. His areas of specialization included Digital Signal and Image Processing and their applications in remote sensing and satellite imagery. He did his Masters under the supervision of Dr. Sergio Cabrera. During his Master's Degree, he published 2 conference papers in IEEE and SPIE. In Spring 2012, he got the acceptance to pursue his $\mathrm{PhD}$ in the same school, he did his

research work with Dr. Miguel Veles-Reyes where he published 3 papers all in IEEE; currently he is working as a lecturer in academic support at Abu Dhabi Polytechnic, UAE.

Contact Information: mqalkhatib@ieee.org

This dissertation was typed by Mohammed Q. Alkhatib 